Universidade de São Paulo

Instituto de Física

\title{
Estudo teórico de nanoestruturas magnéticas em superfícies metálicas
}

\author{
Ricardo Noboru Igarashi \\ Orientador: Profa. Dra. Helena Maria Petrilli \\ Co-orientadora: Dra. Ângela B. Klautau \\ Tese apresentada ao Instituto \\ de Física para a obtenção do \\ título de Doutor em Ciências
}

\section{Comissão Examinadora:}

Profa. Dra. Helena Maria Petrilli (IFUSP)

Prof. Dr. Luiz Gregório Dias da Silva (IFUSP)

Prof. Dr. Mario Norberto Baibich (IF-UFRGS)

Prof. Dr. Gerardo Guido Martínez Pino (IF-UFRGS)

Prof. Dr. Edson Passamani Caetano (DFIS-UFES)

São Paulo

2012 
FICHA CATALOGRÁFICA

Preparada pelo Serviço de Biblioteca e Informação do Instituto de Física da Universidade de SãoPaulo

Igarashi, Ricardo Noboru

Estudo teórico de nanoestruturas magnéticas em superfícies metálicas. - SãoPaulo, 2012.

Tese (Doutorado) - UniversidadedeSãoPaulo

Instituto de Física, Depto.de Física dos Materiais e Mecânica

Orientador: Prof ${ }^{a}{ }^{a}{ }^{a}$ Helena Maria Petrilli Co-Orientador: Prof ${ }^{a}{ }^{a}{ }^{a}$ Ângela Burlamaqui Klautau Área de Concentração: Física do Estado Sólido

Unitermos: 1.Magnetismo; 2.Fenômenos magnéticos; 3.Ferromagnetismo; 4. Ferrimagnetismo.

USP/IF/SBI-082/2012 


\section{Agradecimentos}

Em especial, agradeço a Profa. Helena M. Petrilli por me aceitar como aluno de doutorado no Instituto de Física da Universidade de São Paulo. Isto foi muito importante para mim, pois na USP tive oportunidades de conhecer muitas coisas novas (não apenas na física), que serão muito boas para a minha formação profissional e pessoal. Também agradeço a Profa. Helena por sua paciência, amizade, ensinamento e motivação nestes anos de estudo.

Em especial, agradeço a Profa. Ângela B. Klautau pela sua paciência comigo no ensinamento do método RS-LMTO-ASA, me possibilitando a realização deste trabalho. Também agradeço a Profa. Ângela pela sua hospitalidade quando estive na Universidade Federal do Pará.

A Profa. Marilia Caldas que me recebeu no grupo Nanomol.

Aos professores da banca examinadora pela dedicação na tarefa de avaliar o conteúdo do texto e oferecer suas valiosas contribuições.

Aos professores da Universidade Estadual de Maringá, em especial, Prof. Luis R. Evangelista, Ervin K. Lenzi, Hatsumi Muka e Hercilia A. Pereira que sempre me incentivaram e contribuíram muito no meu início da vida científica.

A Sandra Regina, Rosana Biz e Marisa Fernandes pela ajuda administrativa.

Aos meus amigos do grupo Nanomol, que me ajudaram direta e indiretamente neste trabalho: Ney Sodré, Marcos Brown, Filipe e Phil, Rafael Nascimento, Gabriel Marinello, Jaerlesson Amazonas, Regina Souza, Rodrigo Ramos, Leonardo Jorge, Evandro Jr e José Pinheiro.

Aos amigos que fiz na UFPA, que me ajudaram no entendimento do RSLMTO-ASA: Marcelo Ribeiro, Manoel Neto e Débora Rodrigues.

Aos companheiros do condomínio Vila Gomes: Vagner Rigo (mestre), Joelson Garcia, Dimas Romano, Rolando Larico, Everton Frigo e André Luis.

Em especial, agradeço a minha família: Midori Sakamoto Igarashi, Tadashi Igarashi, Luciana Igarashi e Marcos Mafra, que sempre me apoiaram na minha vida profissional. 
Ao LCCA pelo suporte computacional.

À CAPES e ao CNPQ pelo suporte financeiro. 


\section{Resumo}

Neste trabalho, utilizamos o método de primeiros princípios RS-LMTOASA (Real Space - Linear Muffin-Tin Orbital - Atomic Sphere Approximation), baseado na Teoria do Funcional da Densidade (DFT - Density Functional Theory) e implementado para o cálculo de estruturas magnéticas não colineares, para investigar as propriedades magnéticas de nanoestruturas adsorvidas em superfícies metálicas. Primeiramente, devido ao aparecimento de estruturas magnéticas complexas, foram estudadas nanoestruturas magnéticas depositadas em substratos ferromagnéticos. Foram consideradas nanoestruturas de Mn tais como nanofios adsorvidos, pirâmides e aglomerados adsorvidos e embebidos, com o tamanho destas nanoestruturas variarando de dois até nove átomos depositados em Fe(001) e Fe(110). Nossos cálculos indicam uma interação de troca magnética de longo alcance entre os átomos de Mn-Mn e Mn-Fe. Além disto, a presença de uma forte dependência destas interações de troca magnética com o meio local, frustrações magnéticas e o acoplamento spin-órbita forneceram a possibilidade da presença de estruturas magnéticas complexas tais como, por exemplo, spin espiral e half-skyrmion. Por fim, com o objetivo de estudar nanoestruturas que podem apresentar altos valores de momento magnético, foram investigados nanofios de $\mathrm{Fe}_{x} \mathrm{Co}_{1-x}$ adsorvidos em uma superfície de $\mathrm{Pt}(111)$. Nossos resultados indicam que os momentos magnéticos de spin dos átomos de Fe e Co são independentes da concentração de Fe e apresentam valores superiores quando comparados aos das ligas FeCo bcc, enquanto que o momento magnético médio de spin do nanofio $\mathrm{Fe}_{x} \mathrm{Co}_{1-x}$ varia linearmente com a concentração de Fe, comportamento este que é diferente da curva de SlaterPauling observada na liga FeCo bcc. O momento magnético orbital médio do nanofio de $\mathrm{Fe}_{x} \mathrm{Co}_{1-x}$ é monotonicamente decrescente com a concentração de $\mathrm{Fe}$ que se apresenta bastante diferente a monocamada $\mathrm{Fe}_{x} \mathrm{Co}_{1-x}$ sobre $\operatorname{Pt}(111)$. 


\begin{abstract}
We use the first principles RS-LMTO-ASA (Real Space - Linear MuffinTin Orbital - Atomic Sphere Approximation) method, in the framework of the Density Functional Theory and implemented to calculate noncollinear magnetic structures, to investigate the magnetic properties of nanostructures adsorbed on metallic surfaces. First, due to presence of the complex magnetic properties, we investigated magnetic nanostructures deposited on a ferromagnetic substrate. We have considered a variety of nanostructures such as adsorbed wires, pyramids, flat and intermixed clusters with sizes varying from two to nine atoms deposited on $\mathrm{Fe}(001)$ and $\mathrm{Fe}(110)$. Our calculations reveal the long-range nature of exchange interactions between $\mathrm{Mn}-\mathrm{Mn}$ and Mn-Fe atoms. Moreover, the presence of the strong dependence of these interactions on the local environment, the magnetic frustration, and the effect of spin-orbit coupling lead to the possibility of realizing complex noncollinear magnetic structures such as helical spin spiral and half-skyrmion. Finally, we also investigated $\mathrm{Fe}_{x} \mathrm{Co}_{1-x}$ nanowires deposited on $\mathrm{Pt}(111)$ surface aiming to investigate materials with large local magnetic moment. Our results reveal that the Fe and Co spin magnetic moment are independent of the Fe concentration with the enhancement of the spin magnetic moment when compared with the FeCo bcc alloys, while the average spin magnetic moment is a linear function of the Fe concentration. This is in contrast to the Slater-Pauling model observed in the FeCo bcc alloys. The average orbital magnetic moment shows a linearly decreasing behavior with the Fe concentration which is in contrast to the behavior of $\mathrm{Fe}_{x} \mathrm{Co}_{1-x}$ monolayer on $\mathrm{Pt}(111)$ surface.
\end{abstract}




\section{Conteúdo}

Agradecimentos $\quad$ i

Resumo iii

Abstract iv

1 Introdução 1

2 RS-LMTO-ASA $\quad 6$

2.1 Teoria do Funcional da Densidade . . . . . . . . . . . 6

2.1.1 Sistemas com polarização de spin . . . . . . . . . 8

2.1.2 Acoplamento Spin-Órbita . . . . . . . . . . 9

2.2 Método RS-LMTO-ASA . . . . . . . . . . . . . . . . 9

2.3 Implementação computacional do RS-LMTO-ASA . . . . . . . . . . . . . . . 11

2.3.1 Parte geral . . . . . . . . . . . . . . 11

2.3.2 Parte atômica . . . . . . . . . . . . . . 13

2.3.3 Processo autoconsistente para sistemas metálicos bidimensionais no RS-LMTO-ASA . . . . . . . . . . 14

2.3.4 Processo autoconsistente para defeito em superfície no RS-LMTO-ASA . . . . . . . . . 16

3 Ordenamento Magnético $\quad 19$

3.1 Tipos de ordenamento magnético . . . . . . . . . . . . 19

3.2 Interação de troca magnética . . . . . . . . . . . . . . . 20 
3.2.1 Interação de Dzyaloshinskii-Moriya . . . . . . . . . . 21

3.3 Cálculo da interação de troca com o RS-LMTO-ASA . . . . . 22

3.4 Ocorrência do magnetismo não colinear . . . . . . . . . . . . 23

3.4 .1 Frustração . . . . . . . . . . . . . . . . . . 24

3.4.2 Competição entre interações de troca FM e AF . . . . 25

3.5 Cálculo do magnetismo não colinear . . . . . . . . . . . . . 25

4 Propriedades Magnéticas de Nanoestruturas de Mn Sobre a Superfície Fe(001) 29

4.1 Preâmbulo . . . . . . . . . . . . . . . . . . . . . . . . 29

4.2 Detalhes computacionais . . . . . . . . . . . . . . 30

4.3 Estrutura eletrônica do Fe bcc bulk e da superfície Fe(001) . . 32

4.3 .1 Fe bulk . . . . . . . . . . . . . . . . . . 32

4.3.2 Superfície Fe(001) . . . . . . . . . . . . . 33

4.4 Sistemas unidimensionais de Mn sobre a superfície de Fe(001) 37

4.4.1 Adátomo de Mn sobre a superfície Fe(001) . . . . . . 37

4.4.2 Átomo de Mn embebido na superfície de Fe(001) . . . 40

4.4.3 Nanofios de Mn sobre a superfície Fe(001) - Resultados colineares . . . . . . . . . . . . . . . 42

4.4.4 Efeitos de relaxação . . . . . . . . . . . . . . . . . . 48

4.4.5 Efeitos de Interdifusão . . . . . . . . . . . . . . . . . . 49

4.4.6 Resultados não colineares . . . . . . . . . . . . . 50

4.5 Nanoestruturas bi-tridimensionais de Mn sobre a superfície de $\mathrm{Fe}(001) \ldots \ldots \ldots \ldots \ldots \ldots \ldots$

5 Propriedades magnéticas de nanofios de Mn sobre a superfície $\mathrm{Fe}(110) \quad 59$

5.1 Preâmbulo . . . . . . . . . . . . . . . . . . . . . 59

5.2 Detalhes computacionais . . . . . . . . . . . . . 60

5.3 Nanofios de Mn sobre a superfície Fe(110) . . . . . . . . . 60

5.3.1 Estuturas colineares . . . . . . . . . . . . . 60

5.3 .2 Resultados não colineares . . . . . . . . . . 65 
6 Propriedades magnéticas em cadeias lineares de $\mathrm{Fe}_{x} \mathrm{Co}_{1-x}$ de$\begin{array}{ll}\text { positadas sobre } \operatorname{Pt}(111) & 68\end{array}$

6.1 Preâmbulo . . . . . . . . . . . . . . . . . . . 68

6.2 Detalhes Computacionais . . . . . . . . . . . . 71

6.3 Resultados e Discussões . . . . . . . . . . . . . . . . 73

6.3.1 Momentos magnéticos . . . . . . . . . . . 73

6.3.2 Interação de troca magnética . . . . . . . . . . . 75

6.3.3 Contribuição da polarização orbital . . . . . . . . . . . 77

6.3.4 Análise de estrutura eletrônica . . . . . . . . . . . 78

$\begin{array}{llr}7 & \text { Conclusões } & 82\end{array}$

A Orbitais Lineares Muffin-tin $\quad 84$

A.1 Introdução . . . . . . . . . . . . . . . . . . . . . 84

A.2 O conjunto de bases LMTO . . . . . . . . . . . . 86

A.2.1 Obtenção da Base Canônica . . . . . . . . . . . . . 86

A.2.2 Aproximação da esfera atômica . . . . . . . . . . . 87

A.2.3 Formalismo LMTO-ASA numa Base Genérica . . . . . 92

A.2.4 Base Localizada - TB . . . . . . . . . . . . . . . 94

A.2.5 Base Ortogonal . . . . . . . . . . . . . . . 95

A.2.6 Representação Ortogonal da Matriz Hamiltoniana Expressa em Termos de Parâmetros da Representação Tight-Binding . . . . . . . . . . . . . 97

B Método de Recorrência 100 


\section{Capítulo 1}

\section{Introdução}

A área de física dos materiais tem sido fortemente impulsionada na direção da investigação de propriedades relacionadas a materiais nanoestruturados, tanto do ponto de vista teórico como experimental. Um material nanoestruturado é definido como um sistema que tem, no mínimo, uma dimensão em escala nanométrica (1-100 nm), que pode ser manipulado artificialmente com técnicas experimentais. Dentro deste contexto, multicamadas e aglomerados de átomos depositados em superfícies são considerados materiais nanoestruturados. O desafio nesse campo é tentar compreender e explicar novas propriedades que aparecem em sistemas nanométricos, que não existem em sistemas macroscópicos. Além disso, estas novas características podem ser utilizadas em aplicações tecnológicas como, por exemplo, o efeito de magnetorresistência gigante (GMR) [1] (início dos anos 90) observada em camadas metálicas, e comercialmente usadas em dispositivos de gravação magnética. A descoberta impulsionou o estudo de propriedades magnéticas em sistemas nanométricos, chamado de nanomagnetismo [2]. A importância da pesquisa em nanomagnetismo é que efeitos quânticos representam novos papéis originando diferentes funcionalidades. Por exemplo, as interações magnéticas são drasticamente diferentes para sistemas nanoestruturados quando comparados aos respectivos bulk [3-15].

Um dos grandes interesses no campo do nanomagnetismo é a interação magnética entre um antiferromagneto e um ferromagneto, características dos 
principais constituintes de dispositivos de armazenamento magnético que funcionam usando o efeito de exchange-bias. Entretanto, um completo entendimento microscópico do mecanismo que ocorre na interface é ainda um desafio, devido à presença de vários tipos de ordenamentos químicos e desordem magnética. Neste contexto, camadas de Mn sobre a superfície Fe(100) são sistemas interessantes para investigar acoplamentos magnéticos através de uma interface ferromagneto-antiferromagneto, uma vez que exibem uma intricada dependência entre magnetismo e a espessura dos filmes. Por exemplo, no caso de uma única camada de Mn depositada sobre Fe(001) verifica-se que os momentos magnéticos dos átomos de Mn tem uma estrutura antiferromagnética não colinear no plano, onde os momentos magnéticos do Mn são aproximadamente perpendiculares com a magnetização do Fe [16]. Este resultado está de acordo com os pequenos valores observados experimentalmente para a magnetização da camada de Mn [16-18]. Por outro lado, para sub-monocamadas de Mn em Fe(001), investigações experimentais mostram que os momentos magnéticos dos Mn acoplam-se ferromagneticamente, mas são antiparalelos à magnetização do Fe [17-19]. Do ponto de vista teórico, a estrutura magnética de filmes finos de Mn adsorvidos em Fe(001) tem recebido muita atenção na literatura [16,20-29]. Entretanto, poucos trabalhos investigaram aglomerados (cluster) de Mn em uma superfície Fe(001) cúbica de corpo centrado $(b c c)[22,30]$. Com o avanço dos métodos experimentais nanofios e nanoestruturas podem ser depositadas em superfícies magnéticas e não magnéticas de um modo controlado, e suas propriedades magnéticas fundamentais podem ser exploradas [31-37]. As interações magnéticas na interface Mn e Fe têm assim uma rica variedade, dependendo da geometria e espécies químicas locais. Particularmente, o estudo de nanoestruturas de Mn depositadas em Fe é fascinante.

Neste trabalho, atualmente nos dedicamos ao estudo de nanoestruturas magnéticas metálicas por ser um estimulante campo de pesquisa, explorando suas propriedades magnéticas por meio de abordagens baseadas em métodos de primeiros princípios (ab-initio), utilizando a Mecânica Quântica dentro do esquema de Kohn-Sham para a Teoria do Funcional da Densidade (DFT 
- Density Functional Theory) [38]. Utilizamos o método Real-Space Linear Muffin-Tin Orbital - Atomic Sphere Approximation (RS-LMTO-ASA) [39], que é baseado no método LMTO-ASA na representação Tight-Binding (TB) e foi desenvolvido pela Profa. Sonia Frota-Pessôa (Instituto de Física da Universidade de São Paulo) em uma abordagem no espaço direto para o cálculo de propriedades locais em diferentes sistemas metálicos. Recentemente, novos desenvolvimentos importantes foram implementados nos códigos RS-LMTOASA, tais como: tratamento de momentos magnéticos não colineares, momentos orbitais, entre outros [40,41]. O RS-LMTO-ASA é uma ferramenta bastante poderosa para o tratamento do magnetismo em sistemas nanoestruturados como nano-aglomerados metálicos embebidos em superfícies e em matrizes metálicas $[40,42]$.

Neste trabalho, realizamos cálculos $a b$ initio ${ }^{1}$ de estrutura eletrônica e propriedades magnéticas de nanofios, bem como nano-ilhas bidimensionais e tridimensionais de $\mathrm{Mn}$ sobre $\mathrm{Fe}(001)$. O objetivo é entender quais são estas interações magnéticas e como explicar o magnetismo dependente da espessura da monocamada de Mn em Fe(001). Nossa investigação também é motivada por uma possível existência de configurações magnéticas bi-estáveis ou com múltiplas estabilidades, com energias totais muito próximas, diferindo de alguns meV [43]. Este tipos de nanoestruturas magnéticas são candidatos promissores para unidades de armazenamentos de dados em nanoescala; recentes estudos teóricos exploram a possibilidade de usar campos elétricos [44] ou magnéticos [45] para mudar a configuração magnética de um estado estável para outro. Uma compilação dos resultados por nós obtidos para nanoestruturas de Mn depositadas em Fe(001) foram já publicados [46].

Motivados pelos resultados obtidos para nanofios de Mn em Fe(001), investigamos as propriedades eletrônicas e magnéticas de nanofios de Mn em uma superfície bcc de Fe(110). Neste caso é diferente a quantidade de vizinhos Fe de cada Mn (pela mudança estrutural), o que possibilita o aparecimento de propriedades magnéticas diferentes das de nanofios de Mn em

\footnotetext{
${ }^{1}$ Utilizaremos ao longo deste trabalho o termo ab initio para designar cálculos de estrutura eletrônica no esquema de Kohn-Sham para a DFT.
} 
$\mathrm{Fe}(001)$. Este fato foi verificado por resultados experimentais de monocamadas de Mn em Fe(110) [47], que indicam que a magnetização é zero para sub-monocamadas de $\mathrm{Mn}(0,3 \mathrm{Mn}$ monolayers $(M L))$. Porém, para 0,4 $M L$ até 2,0 $M L$ aparece uma magnetização resultante de $(1,0 \pm 0,2) \mu_{B}$. Desta forma, neste sistema, investigamos como varia o magnetismo dos nanofios de Mn quando a direção do substrato é modificada.

Consideramos então nanoestruturas metálicas com altos valores de momento magnético para possíveis aplicações em meios de gravação magnética (hard disk drive).A liga bcc FeCo é um promissor sistema devido ao fato de que resultados experimentais [48] e teóricos [49,50] mostrarem o momento magnético médio da liga segue a curva de Slater-Pauling, com um máximo em torno de 2,4 $\mu_{B}$ em $\approx 30 \%$ de Co [51]. O desafio, neste caso, é construir sistemas artificiais de FeCo que apresentem momentos magnéticos que superem os valores do bulk. Uma maneira sugerida na literatura é crescer sistemas, como por exemplo, estruturas com distorções de rede [52], multicamadas [53-55] e aglomerados depositados em superfícies [56]. Um resultado interessante é para uma monocamada de $\mathrm{Fe}_{x} \mathrm{Co}_{1-x}$ depositada em $\mathrm{Pt}(111)$, onde resultados teóricos e experimentais mostram altos valores de momento [53] em comparação com o bulk bcc FeCo.

Motivados a encontrar sistemas que possuem altos valores de momento magnético realizamos cálculos ab-initio para investigar as propriedades eletrônicas e magnéticas de cadeias lineares de $\mathrm{Fe}_{x} \mathrm{Co}_{1-x}$ depositadas em uma superfície $\mathrm{Pt}(111)$. As diferenças entre os nossos resultados de nanofios de $\mathrm{Fe}_{x} \mathrm{Co}_{1-x}$ com os resultados experimentais e teóricos para filmes de $\mathrm{Fe}_{x} \mathrm{Co}_{1-x}$ depositados em uma superfície Pt(111) [53] foram também exploradas. Estes nanofios podem apresentar comportamentos magnéticos complexos em conseqüência de que efeitos magnéticos são usualmente mais pronunciados conforme o número de coordenação diminui. Por exemplo, resultados teóricos para nano-aglomerados de FeCo depositados em $\mathrm{Cu}(001)$ indicam que o valor do momento magnético dos átomos de Fe e Co depende da suas posições dentro do aglomerado, isto é, do tipo e também do número de primeiros vizinhos [56]. A direção preferencial de magnetização de um particular nano- 
aglomerado de $\mathrm{FeCo}$ em $\mathrm{Cu}(001)$ é uma resultante da interação entre vários átomos constituintes do aglomerado. Nestes nanofios, nos concentramos na investigação dos efeitos de composição sobre os momentos magnéticos dos átomos de Fe e Co sobre Pt(111). Foi discutida também a interação de troca magnética entre os átomos constituintes das cadeias lineares e sua dependência com o meio local, por exemplo, com o número e tipo de vizinhos que cada átomo de Fe e Co possuem. Também discutimos a dependência dos momentos magnéticos dos átomos de Fe e Co com o meio local.

Este trabalho está organizado da forma descrita a seguir: no Capítulo 2 apresentamos o método RS-LMTO-ASA e no Capítulo 3 os tipos de ordenamento magnético. Os resultados e discussões obtidos para os sistemas constituídos por nanoestruturas de Mn sobre o Fe(001) são apresentados no Capítulo 4 e no Capítulo 5 os resultados para nanofios de Mn em Fe(110). Os resultados obtidos para nanofios de $\mathrm{Fe}_{x} \mathrm{Co}_{1-x}$ em $\mathrm{Pt}(111)$ são apresentados no Capítulo 6. Por fim, no Capítulo 7 foram apresentadas as nossas conclusões. Vale ressaltar que cada um dos sistemas estudados neste trabalho tem uma vasta literatura e por isto, para maior clareza, uma pequena introdução com as informações relevantes foi adicionado a cada capítulo. 


\section{Capítulo 2}

\section{RS-LMTO-ASA}

\subsection{Teoria do Funcional da Densidade}

O objetivo de um método de cálculo de estrutura eletrônica de sólidos é resolver a equação de Schrödinger:

$$
\hat{H} \Psi=E \Psi
$$

onde $E$ é a energia total do sistema. A Hamiltoniana $H$ contém a energia cinética dos núcleos e elétrons, as interações elétron-elétron, núcleo-elétron e núcleo-núcleo. Nesta situação a equação de Schrödinger torna-se muito complicada de ser resolvida. Assim, algumas aproximações devem ser adotadas para o cálculo das funções de onda e autovalores. A primeira leva em consideração de que a massa dos elétrons é muito menor do que a massa do núcleo. Portanto, o termo cinético dos elétrons é bem maior que o termo nuclear, possibilitando o desacoplamento da Eq. (2.1) em uma parte eletrônica e outra nuclear. Esta aproximação é chamada de Born-Oppenheimer ou adiabática [57]. Outra aproximação necessária é referente ao problema de vários corpos, uma vez que o sistema é formado por um número grande de elétrons interagentes. Uma alternativa para a resolução deste problema vem com o desenvolvimento da Teoria do Funcional da Densidade (DFT) por Hohenberg e Kohn [58], onde é explorada a densidade eletrônica, $n(\vec{r})$, estabelecendo que o estado fundamental de um sistema é obtido pela mini- 
mização do funcional energia $E[n]$. A partir da teoria de Hohenberg-Kohn foi desenvolvida uma formulação matemática para a DFT chamada de esquema de Kohn-Sham (KS) [38]. Neste procedimento, o estado fundamental é encontrado pela resolução de um conjunto de equações tipo-Schrödinger para cada elétron (chamadas também de equações de KS)

$$
\left[-\nabla^{2}+V_{e f f}(\vec{r})\right] \psi_{i}(\vec{r})=\varepsilon_{i} \psi_{i}(\vec{r})
$$

Aqui $\psi_{i}$ é a função de onda para o elétron $i$ com uma energia $\varepsilon_{i}$, e o potencial $V_{\text {eff }}$ é escrito como:

$$
V_{e f f}=V_{e}+V_{N}+V_{C T}
$$

onde $V_{e}$ é o potencial devido aos outros elétrons do sistema, $V_{N}$ é o potencial dos núcleos, e $V_{C T}$ é chamado potencial de troca e correlação. Todos os efeitos de muitos corpos do problema original são agora incorporados no termo de troca e correlação, sendo necessário também utilizar algumas aproximações para este termo. Uma possibilidade é tratar o potencial de troca e correlação como um potencial de um gás homogêneo de elétrons interagentes, chamada Aproximação da Densidade Local (LDA - Local Density Approximation) e é provavelmente a aproximação mais comum para a troca e correlação, sendo bastante eficiente para tratar sistemas onde a densidade varia lentamente. Quando o sistema apresenta polarização de spin, como será apresentado na próxima seção, usa-se a Aproximação da Densidade Local por Spin (LSDA Local Spin Density Approximation) [59]. Quando se trata o potencial de troca e correlação como função não apenas da densidade eletrônica, mas também do gradiente da densidade, temos a chamada Aproximação do Gradiente Generalizado (GGA - Generalized Gradient Approximation) [60]. Além da LDA e GGA existem outros tipos de parametrizações, como por exemplo, cálculos Monte Carlo, etc. No presente trabalho foi usada a LSDA proposta por von Barth e Hedin [59]. 


\subsubsection{Sistemas com polarização de spin}

Para tratar a densidade de elétrons com spin polarizados na LSDA a densidade eletrônica $n(\vec{r})$ é substituída por uma matriz densidade, $\rho(\vec{r})$, escrita como:

$$
\rho(\vec{r})=\frac{n(\vec{r})}{2} \mathbf{1}+\frac{\vec{m}(\vec{r})}{2} \sigma,
$$

onde 1 é a matriz unitária $2 \times 2, \vec{m}(\vec{r})$ é a densidade de magnetização e $\sigma=\left(\sigma_{x}\right.$, $\left.\sigma_{y}, \sigma_{z}\right)$ são as matrizes de Pauli. As funções de onda correspondentes, $\psi_{i}(\vec{r})$, são representadas pelos espinores na forma:

$$
\psi_{i}=\left(\begin{array}{c}
\alpha_{i} \\
\beta_{i}
\end{array}\right),
$$

onde $\alpha_{i}(\vec{r})$ e $\beta_{i}(\vec{r})$ são projeções de spin. A matriz densidade é expressa em termos dos espinores como:

$$
\rho(\vec{r})=\sum_{i}^{N}\left(\begin{array}{cc}
\left|\alpha_{i}(\vec{r})\right|^{2} & \alpha_{i}(\vec{r}) \beta_{i}(\vec{r})^{*} \\
\alpha_{i}(\vec{r})^{*} \beta_{i}(\vec{r}) & \left|\beta_{i}(\vec{r})\right|^{2}
\end{array}\right) .
$$

As densidades de carga e magnetização são expressas da seguinte forma:

$$
\begin{gathered}
n(\vec{r})=\operatorname{Tr}(\rho(\vec{r}))=\sum_{i}^{N}\left|\psi_{i}(\vec{r})\right|^{2} \\
\vec{m}(\vec{r})=\sum_{i}^{N} \psi_{i}(\vec{r})^{\dagger} \sigma \psi_{i}(\vec{r}) .
\end{gathered}
$$

De maneira análoga à densidade eletrônica, o potencial externo é expandido em uma matriz $2 \times 2$ e as equações de Kohn-Sham [Eq. (2.2)] podem ser generalizadas para sistemas com polarização de spin, na forma:

$$
\sum_{\beta}\left[-\delta_{\alpha \beta} \nabla^{2}+V_{e f f}^{\alpha \beta}(\vec{r})\right] \psi_{i \beta}=\epsilon_{i} \delta_{\alpha \beta} \psi_{i \beta}(\vec{r}) \quad \alpha=1,2 .
$$

O potencial efetivo $V_{\text {eff }}(\vec{r})$ ) pode ser decomposto em uma parte magnética, b, e uma parte não magnética, $v_{N M}$. Desta forma, a Hamiltoniana de KohnSham para um sistema dependente de spin pode ser escrita na LSDA como:

$$
H_{S R}=\left(-\nabla^{2}+v_{N M}\right) \mathbf{1}+\mathbf{b} \cdot \sigma,
$$

onde $H_{S R}$ é chamada de Hamiltoniana escalar relativística. 


\subsubsection{Acoplamento Spin-Órbita}

Para calcular momentos orbitais e anisotropias magnéticas, devemos levar em consideração o acoplamento spin-órbita que pode ser adicionado à $H_{S R}$ [61-63] na forma:

$$
H_{F R}=H_{S R}+\xi L \cdot S
$$

obtendo a Hamiltoniana Fully Relativistic $H_{F R}$ e será usada nos cálculos para os cálculos de estrutura eletrônica neste trabalho. Aqui $\xi$ é a intensidade do acoplamento spin-órbita escrita como:

$$
\xi \propto \frac{1}{r} \frac{\partial V}{\partial r}
$$

que é dependente do número atômico. Portanto, os efeitos de spin-órbita são maiores para elementos com maior número atômico. Pode-se também incorporar na Hamiltoniana o efeito de polarização orbital intra-atômica de origem eletrostática, sendo este responsável pela maximização dos momentos orbitais, conforme dita a segunda regra de Hund para átomos livres. Uma vez que este efeito não pode ser descrito pela aproximação LDA, Eriksson et al. [64] sugeriram que o efeito de polarização de orbital (OP - Orbital Polarization) pode ser introduzido adicionando-se à Hamiltoniana um termo de energia dado por:

$$
E_{O P}=-\frac{1}{2} B m_{L}^{2}
$$

onde B é o parâmetro de Racah e $m_{L}$ o momento orbital. Realizamos cálculos com a inclusão de $\mathrm{OP}$ no estudo de nanofios de $\mathrm{Fe}_{x} \mathrm{Co}_{1-x}$ depositados sobre uma superfície $\operatorname{Pt}(111)$.

\section{$2.2 \quad$ Método RS-LMTO-ASA}

Para obtermos a estrutura eletrônica e propriedades magnéticas dos sistemas propostos, usamos o método de primeiros princípios RS-LMTO-ASA baseado no esquema de KS para a DFT e utilizamos a LSDA. O RS-LMTOASA é baseado no formalismo LMTO-ASA no espaço $\vec{k}$, desenvolvido por 
O. K. Andersen $[61,65,66]$. No entanto, este método calcula os autovalores no espaço real usando o Método de Recorrência de Haydock [67] com o terminador de Beer-Pettifor [68]. O RS-LMTO-ASA é um método linear e as soluções das equações de KS são mais precisas em torno de uma energia $E_{\nu}$, usualmente tomada como centro de gravidade das bandas $s, p$ e $d$ ocupadas. O problema de autovalores é escrito como [69-72]:

$$
(H-E) u=0
$$

e a função de onda correspondente é:

$$
\Psi_{E}=\sum_{R L}\left[\varphi_{R l}\left(r_{R}\right)+\left(E-E_{\nu}\right) \dot{\varphi}_{R l}\left(r_{R}\right)\right] Y_{L}\left(\hat{r}_{R}\right) u_{R L}(E)
$$

As funções $\varphi_{R l}\left(r_{R}\right)$ e $\dot{\varphi}_{R l}\left(r_{R}\right)$ são respectivamente as soluções da equação tipo-Schrödinger radial para uma única partícula e sua derivada em relação a energia, calculadas para uma energia $E=E_{\nu}$ dentro de uma esfera de Wigner-Seitz (WS), centrada em um sítio atômico (R). O índice $L$ denota os números quânticos $(l, m)$ e as funções $\varphi_{R l}\left(r_{R}\right)$ e $\dot{\varphi}_{R l}\left(r_{R}\right)$ são nulas fora da esfera de WS. $Y_{L}$ é a função harmônica esférica e $u_{R L}$ são os coeficientes da função de onda.

Uma das vantagens do LMTO-ASA é que pode ser escrito em diferentes representações [69-72] como discutido em detalhes no Apêndice A. A Hamiltoniana na base ortogonal pode ser escrita em termos dos assim chamados parâmetros Tight-Binding (TB) como:

$$
H=E_{\nu}+\bar{h}-\bar{h} \bar{o}
$$

onde $\bar{h}$ é uma matriz hermitiana expressa em termos dos parâmetros TB, dada por (ver Apêndice A):

$$
\bar{h}=\bar{C}-E_{\nu}+\bar{\Delta}^{1 / 2} \bar{S} \bar{\Delta}^{1 / 2} .
$$

Usando a aproximação de primeira ordem em $\bar{h}$ desprezando o termo $\bar{h} \bar{o} \bar{h}$, a Hamiltoniana fica:

$$
H=\bar{C}+\bar{\Delta}^{1 / 2} \bar{S} \bar{\Delta}^{1 / 2}
$$


Aqui $\bar{C}, \bar{\Delta}$ e $\bar{o}$ são parâmetros de potencial e $\bar{S}$ é a constante de estrutura (posições atômicas) na representação TB.

Para uma boa descrição das partes ocupadas das bandas $s, p$ e $d$, a Hamiltoniana de primeira ordem Eq.(2.16) é suficiente. Entretanto, para uma boa descrição dos estados desocupados são necessários os termos de segunda ordem, ou seja, $\bar{h} \bar{o} \bar{h}$ deve ser incluído na Hamiltoniana Eq.(2.14). Para as propriedades magnéticas estudadas neste trabalho, a inclusão do termo de segunda ordem não acarreta diferenças significativas nos resultados. Sendo assim, utilizaremos a Hamiltoniana de primeira ordem, tendo em vista que a Hamiltonina de segunda ordem torna o processo autoconsistente muito mais custoso.

\subsection{Implementação computacional do RS-LMTO-ASA}

A implementação computacional do RS-LMTO-ASA tem duas partes autoconsistentes entrelaçadas esquematizadas nas Figs. 2.1 (parte geral) e 2.2 (parte atômica). Apresentamos a seguir procedimentos autoconsistentes no espaço direto, para a determinação da estrutura eletrônica de sistemas metálicos.

\subsubsection{Parte geral}

Conforme a Fig. 2.1, inicia-se a parte geral com duas etapas independentes: onde uma é calculada a constante de estrutura $\bar{S}$, que depende apenas da estrutura espacial do material e na outra parte são calculados os parâmetros de potencial $\bar{C}$ e $\bar{\Delta}$. Inicialmente, calculamos os parâmetros de potencial na base ortogonal que são convertidos para a base TB, usando a seguinte relação:

$$
\frac{\Delta^{1 / 2}}{\bar{\Delta}^{1 / 2}}=1-(\bar{Q}-Q) \frac{\bar{C}-E_{\nu}}{\bar{\Delta}}=\frac{C-E_{\nu}}{\bar{C}-E_{\nu}}
$$




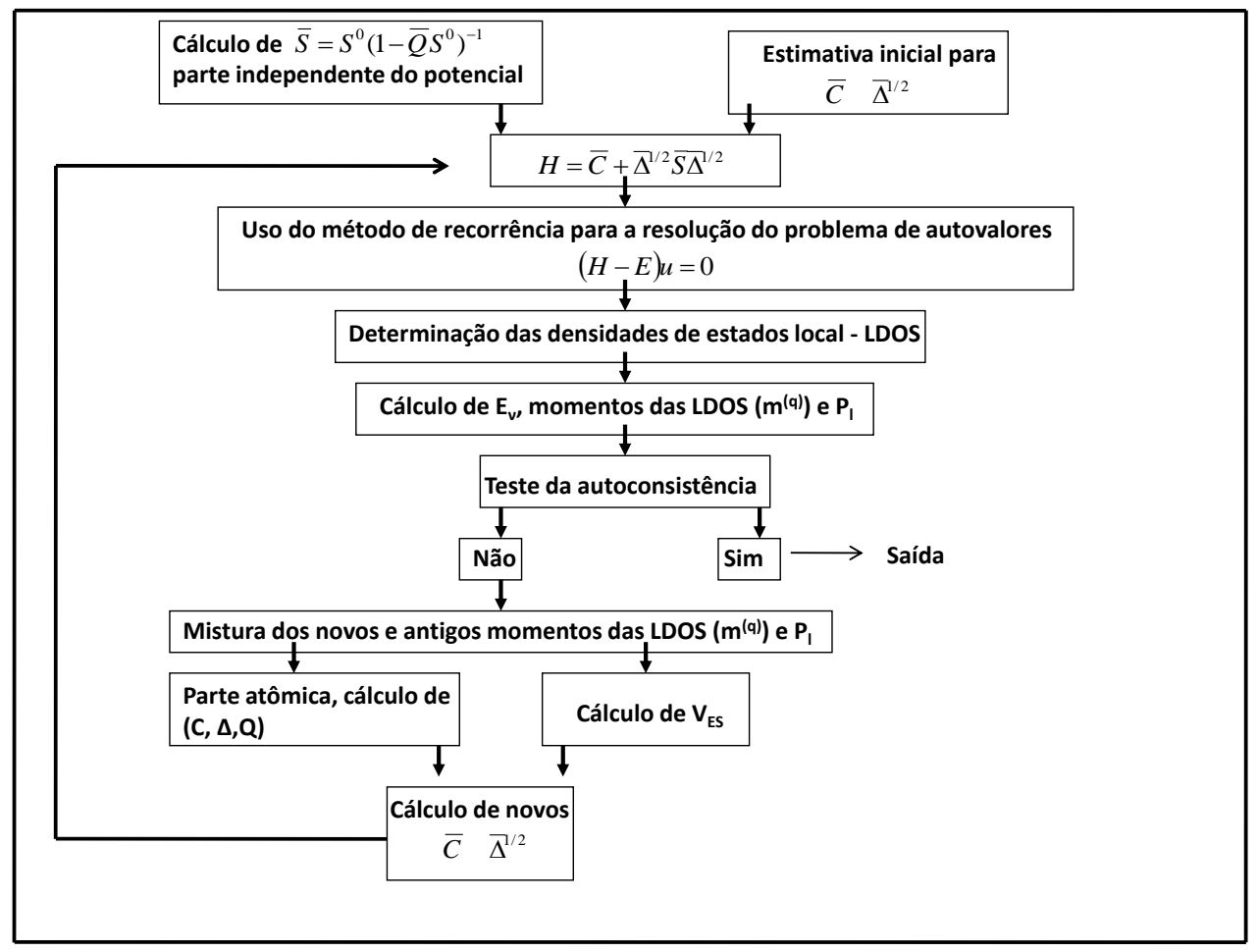

Figura 2.1: Fluxograma relativo ao esquema da parte geral do método RSLMTO-ASA.

onde a notação sem barra representa a base ortogonal. A quantidade $\bar{Q}$ é chamada de parâmetro de mistura, que relaciona a constante de estrutura escrita na base ortogonal com a da base TB, através de

$$
\bar{S}=S(1-\bar{Q} S)^{-1}
$$

Calculada a constante de estrutura do material através de (2.18) e paralelamente é feita uma estimativa inicial de $\bar{C}$ e $\bar{\Delta}$ e é construída a Hamiltoniana. Utilizando o Método de Recorrência são obtidos os autovalores e os momentos das Densidades de Estados Local ( LDOS -Local Density of States) $N_{R L}(E)$ :

$$
m^{(q)}=\int^{E_{F}}\left(E-E_{\nu}\right)^{q} N_{R L}(E) d E,
$$

onde $q=0,1,2$. O momento de ordem zero $\left(m^{0}\right)$ é a ocupação de cada orbital $s, p$ e $d$. Escolhendo a energia $E_{\nu}$ como sendo a energia do centro de 
gravidade da banda ocupada, o momento de ordem um $\left(m^{1}\right)$ é igual a zero. Na parte geral também são obtidas as condições de contorno para cada esfera não equivalente que são definidas através dos parâmetros $P_{l}$ :

$$
P_{l}=0.5-\frac{1}{\pi} \arctan \left(D_{l}\right)
$$

onde $0<P_{l} \leq 1$ e $D_{l}$ é a derivada logarítmica da solução da equação de KohnSham impondo condições de continuidade no contorno da esfera [70]. A parte geral alcança a convergência quando não existirem variações significativas entre os valores novos e antigos dos momentos da LDOS e $P_{l}$ (ver a Fig. 2.1).

\subsubsection{Parte atômica}

Na parte atômica (Fig. 2.2) são obtidos os parâmetros de potencial em cada esfera não equivalente que são usados na parte geral. Inicialmente é feito o cálculo da densidade eletrônica para cada esfera não equivalente usando:

$$
n_{R}\left(r_{R}\right)=\frac{1}{4 \pi} \sum_{l}\left[m_{R l}^{(0)} \varphi_{R l}^{2}+m_{R l}^{(2)}\left(\dot{\varphi}_{R l}+\varphi_{R l} \ddot{\varphi}_{R l}\right)\right]
$$

onde $m_{R l}^{(q)}$ são os momentos dados pela Eq.( 2.19), e os $\varphi_{R l}$ são as soluções das equações tipo-Schrödinger radiais. Com a densidade eletrônica podemos determinar o potencial eletrostático $\left(V_{E}\right)$ através da solução da equação de Poisson. Este potencial é adicionado ao potencial de troca e correlação $\left(V_{C T}\right)$ (obtido usando a LDA) e também à contribuição nuclear $\left(V_{N}\right)$,

$$
V_{e f f}=V_{e}+V_{C T}+V_{N}
$$

Com o potencial $V_{\text {eff }}$, resolvemos a equação tipo-Schrödinger radial para obter as novas funções de onda calculadas em torno da energia $E_{\nu}$, e encontramos os novos parâmetros de potencial $\left(C_{R l}, \Delta_{R l}, Q_{R l}\right)$ e usando a relação (2.17), obtemos os parâmetros na base TB. Este processo é repetido de forma autoconsistente. Paralelamente a estes dois processos é calculado o potencial de Madelung $\left(V_{E S}\right)$ que leva em conta os efeitos das cargas vizinhas e a contribuição eletrônica do próprio sítio. 


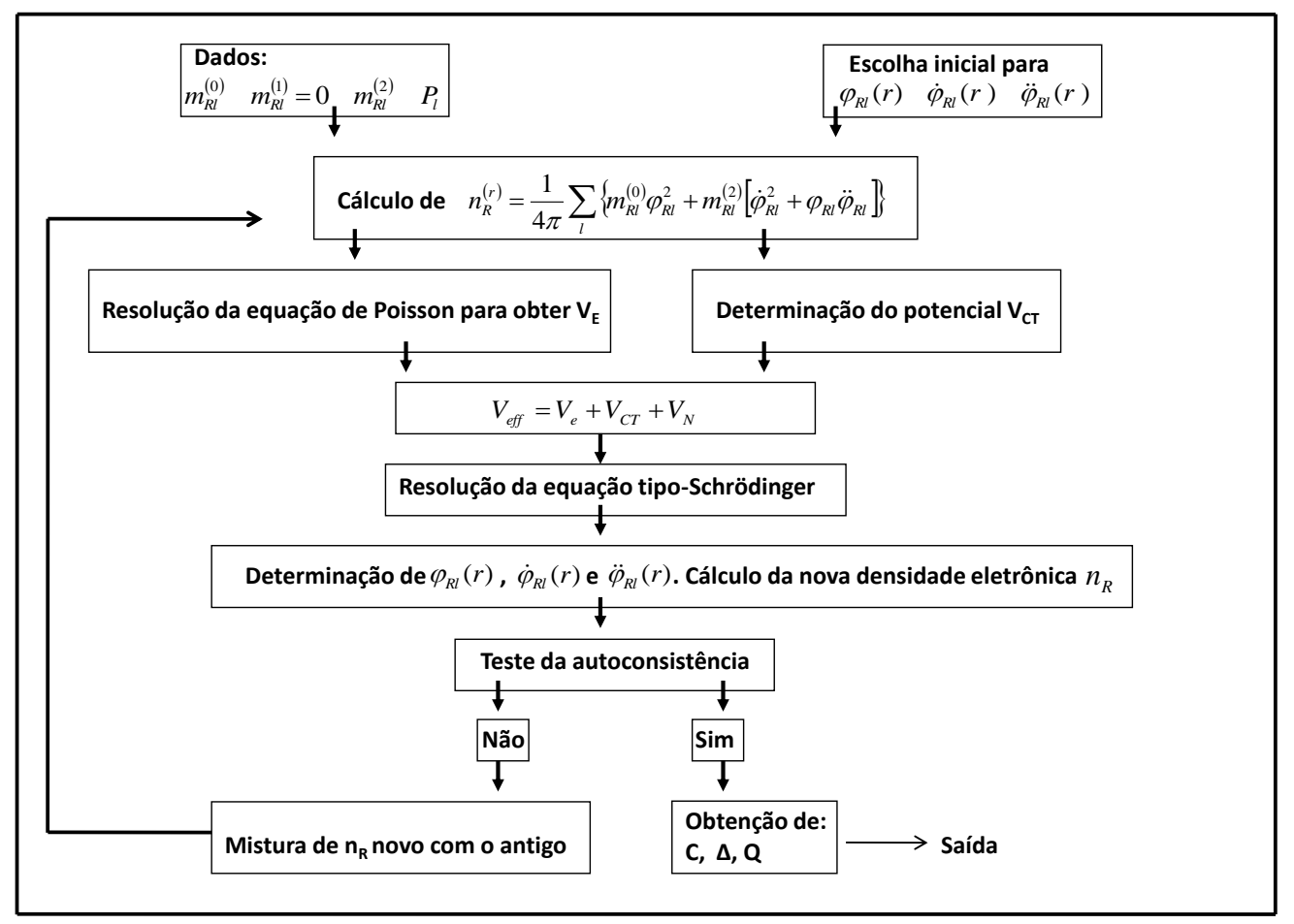

Figura 2.2: Fluxograma relativo ao esquema da parte atômica do método RS-LMTO-ASA.

\subsubsection{Processo autoconsistente para sistemas metálicos bidimensionais no RS-LMTO-ASA}

Nesta seção, descrevemos como calcular sistemas metálicos bidimensionais com o RS-LMTO-ASA. Primeiramente, usamos um aglomerado com aproximadamente 10000 átomos e construímos a superfície na direção que desejamos, por exemplo, (001), (111) ou (110) definindo também a quantidade de camadas incluídas no cálculo autoconsistente.

Para simular a interface com o vácuo em sistemas metálicos bidimensionais, colocamos duas camadas de esferas vazias. A quantidade de carga transferida para as esferas vazias é obtida através do cálculo autoconsistente. Esta região próxima à superfície funciona como um capacitor de placas paralelas, modificando o potencial eletrostático e deslocando a energia de Fermi 
de outras camadas da superfície. Para evitar este deslocamento é redefinida a escala de energia de tal forma que o potencial seja zero em sítios distantes da superfície [71]. Para tanto, subtraímos do potencial de todas as camadas o potencial do material bulk. Fixado o valor da energia de Fermi no valor obtido para o bulk, podemos calcular as transferências de carga e parâmetros de potencial de cada camada. Como estamos tratando de sistemas com simetria bidimensional, usamos a soma de Ewald bidimensional de Skriver et al. $[73,74]$ para a obtenção do potencial eletrostático em cada sítio $V_{E S}$. Também consideramos que as camadas mais distantes da superfície tenham as mesmas propriedades (parâmetros de potencial) do material bulk ${ }^{1}$.

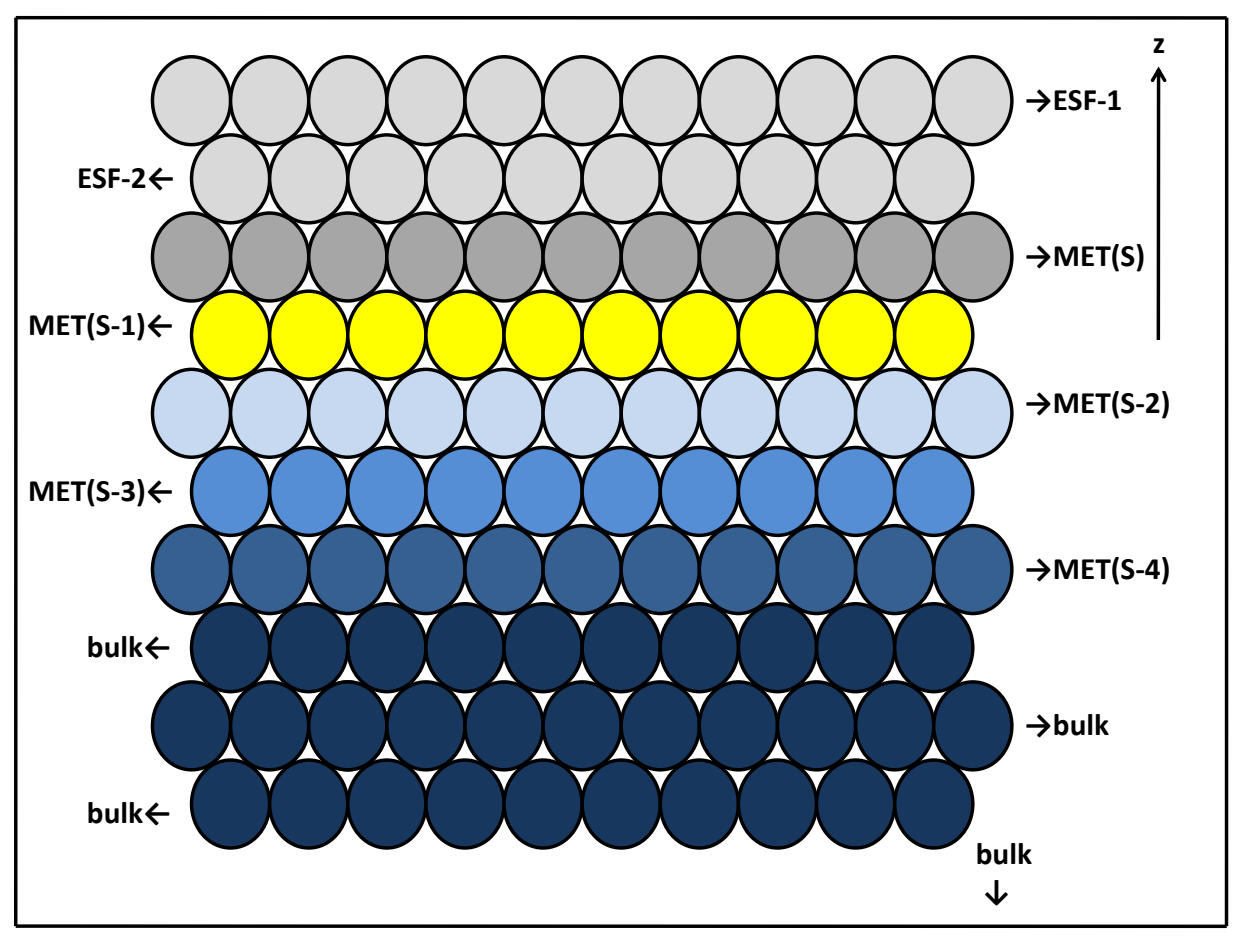

Figura 2.3: Esquema para a superfície livre com duas camadas de esferas vazias (ESF-1, ESF-2) e com cinco camadas metálicas (MET(S), MET(S1), $\operatorname{MET}(\mathrm{S}-2), \operatorname{MET}(\mathrm{S}-3)$ e $\operatorname{MET}(\mathrm{S}-4))$ depositadas sobre o material bulk metálico.

\footnotetext{
${ }^{1} \mathrm{Em}$ todos os nossos cálculos de superfície livre (sem defeitos) é necessário antes fazer o cálculo do material bulk.
} 
Passamos agora a descrição do processo realizado neste trabalho para a obtenção das propriedades da superfície (conforme esquematizado nas Figs. 2.1 e 2.2). Primeiro consideramos as camadas de esfera vazias ESF-1, ESF-2 e a primeira camada metálica $\operatorname{MET}(\mathrm{S})$ para o cálculo autoconsistente (na Fig. 2.3 é mostrado um esquema para uma superfície metálica). O restante do sistema é mantido com os parâmetros de potencial do bulk. Inicialmente é feita uma estimativa para os momentos da densidade de estados e $P_{l}$ destas três camadas para construção da Hamiltoniana. Lembramos que cada camada pode ser representada por um átomo não equivalente. Resolvendo a Eq.(2.12) (seguindo o esquema da Fig. 2.1) obtemos os novos momentos da densidade de estados e as transferências de carga $(\Delta Q)$ para os outros sítios. Quando é alcançada a convergência neste primeiro processo iterativo, podemos incluir mais uma camada justaposta às anteriores. A convergência da superfície livre termina quando a última camada incluída tem os mesmos parâmetros de potencial do material bulk.

\subsubsection{Processo autoconsistente para defeito em superfície no RS-LMTO-ASA}

Inicialmente, tomamos o nível de Fermi do material bulk, o qual não será modificado quando o defeito é introduzido na superfície. Também definimos que os valores dos potenciais eletrostáticos, transferências de cargas e parâmetros de potencial nos sítios distantes do defeito não são significativamente afetados pela presença destes defeitos. Para distinguir as transferências de carga e os potenciais eletrostáticos calculados para a superfície livre e para os sítios na presença de um defeito, utilizamos a seguinte notação: as quantidades $\Delta Q_{\text {sup }}$ e $V_{E S_{\text {sup }}}$ indicam as transferências de carga e os potenciais eletrostáticos obtidos para a superfície livre, enquanto que $\Delta Q$ e $V_{E S}$ designam esses valores na presença de um defeito. Também, definimos as transferências de carga local $\Delta Q_{\text {local }}$ e os potenciais $V_{E S_{\text {local }}}$ associados com as diferenças entre as cargas de transferência $\Delta Q$ e os potenciais $V_{E S} \mathrm{em}$ 
cada sítio, e os seus valores para a superfície livre. Definimos desta forma:

$$
\begin{aligned}
& \Delta Q=\Delta Q_{\text {sup }}+\Delta Q_{\text {local }}, \\
& V_{E S}=V_{E S_{\text {sup }}}+V_{E S_{\text {local }}} .
\end{aligned}
$$

A transferência de carga $\Delta Q$ é escrita como uma soma das transferências de carga $\Delta Q_{\text {sup }}$ na superfície livre e a transferência de carga local $\Delta Q_{\text {local }}$. Uma vez que o potencial eletrostático obedece ao princípio de superposição, os valores de $V_{E S}$, associados à transferência de carga $\Delta Q$, podem ser escritos como uma soma de $V_{E S_{\text {sup }}}$ e $V_{E S_{\text {local }}}$.

Para exemplificar usamos o caso do adátomo (um único átomo adsorvido), contudo, este procedimento pode ser usado para qualquer tipo de defeito. Inicialmente consideramos o arranjo ilustrado na Fig. 2.4 para uma superfície e duas camadas de esferas vazias, onde o defeito é colocado na posição central na camada de esferas vazias (ESF-2). Usamos uma estimativa inicial para os parâmetros de potencial desta impureza para construir sua Hamiltoniana. Este processo é chamado de sítio único, pois os outros átomos ficam com os parâmetros de potencial obtidos da superfície livre. Utilizando a parte geral e atômica do processo autoconsistente, calculamos $\Delta Q$ integrando a LDOS até a energia de Fermi. Usando a Eq.( 2.23) encontramos a transferência de carga local. O excesso de carga é colocado na primeira vizinhança da impureza para obedecer a lei da conservação da carga. Com os valores da $\Delta Q_{\text {local }}$, obtemos o potencial eletrostático local e usando a Eq.(2.24) encontramos $V_{E S}$. Com este valor, calculamos os novos parâmetros de potencial que são misturados com os valores antigos para recomeçar o cálculo. O processo iterativo termina quando obtemos a convergência nas ocupações do adátomo.

Com o cálculo de sítio único convergido incluímos os primeiros vizinhos do adátomo (Fig. 2.4) num processo iterativo . Usamos para os átomos vizinhos do adátomo nesta camada os parâmetros de potencial obtidos no caso da superfície livre, e os parâmetros de potencial do processo de sítio único para o adátomo como estimativa inicial. Os novos parâmetros de potencial desta configuração são obtidos autoconsistentemente como no caso anterior. 


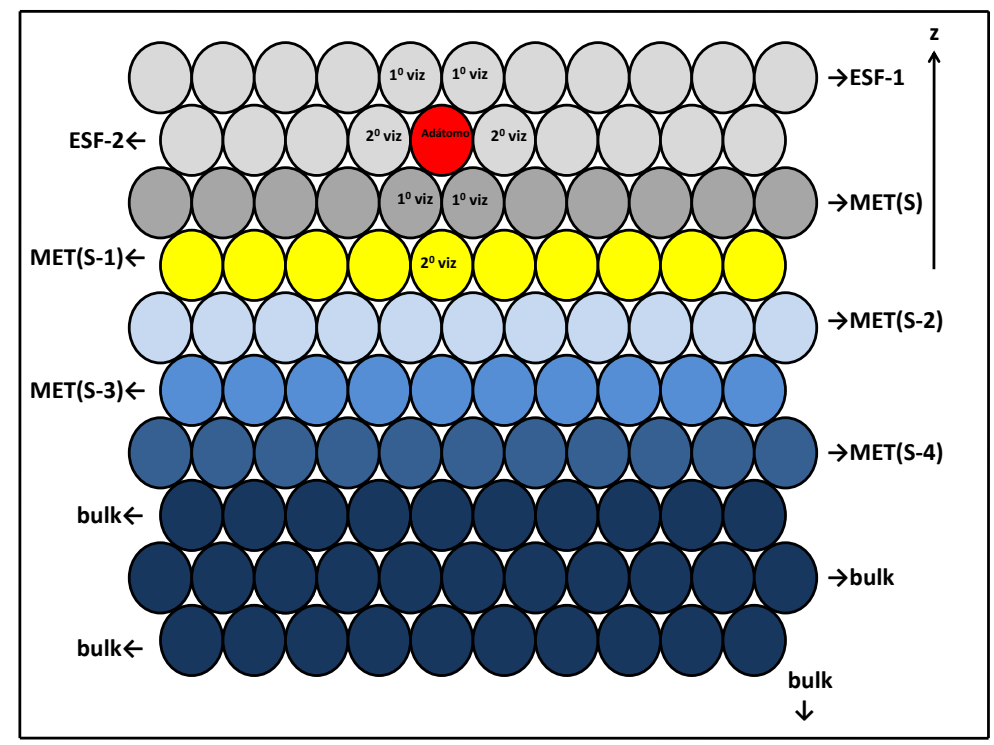

Figura 2.4: Esquema para o cálculo autoconsistente para o caso do adátomo.

O processo de inclusão de novos vizinhos termina quando a última camada incluída tiver as mesmas ocupações que na superfície livre. 


\section{Capítulo 3}

\section{Ordenamento Magnético}

Analogamente ao magnetismo de átomos isolados, que pode ser estudado usando às regras de Hund, os átomos que formam um sólido podem ter seu comportamento magnético analisado através das interações de troca magnética, onde para interações fortes podem resultar em uma variedade de ordenamentos magnéticos. Neste capítulo, apresentamos os vários tipos de ordenamentos magnéticos que podem ocorrer nos sistemas investigados no presente trabalho.

\subsection{Tipos de ordenamento magnético}

Magnetismo colinear é a denominação à situação onde os momentos magnéticos dos átomos se alinham ao longo de um único eixo global de magnetização. Quando estes momentos magnéticos se alinham paralelamente, temos o caso ferromagnético (FM) (Fig. 3.1-a) e quando se alinham antiparalelamente, o caso antiferromagnético (AF) (Fig. 3.1-b). Existe também o caso ferrimagnético (FI), onde os momentos magnéticos são antiparalelos, mas com valores diferentes. As causas do ordenamento FM e AF podem ser previstas com o uso de teorias bem estabelecidas, por exemplo, o modelo de Stoner [75]. Quando os vários momentos magnéticos não estão alinhados ao longo de um único eixo temos o magnetismo não colinear, como ilustrado na Fig. 3.1-c. Neste capítulo, apresentamos algumas situações onde o mag- 
netismo não colinear pode aparecer.

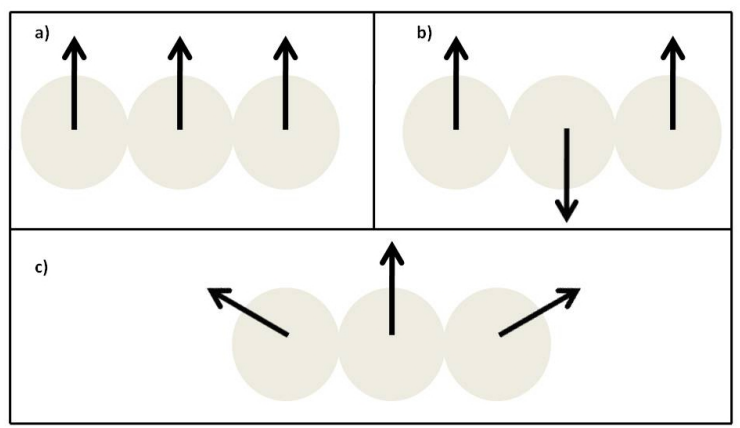

Figura 3.1: Exemplos de ordenamentos magnéticos: a) ferromagnético (FM), b) antiferromagnético (AF) e c) não colinear.

\subsection{Interação de troca magnética}

Para o cálculo da interação magnética entre dois sítios na configuração magnética colinear usualmente é utilizado o modelo de Heisenberg [76] descrito pela Hamiltoniana:

$$
H=-\sum_{i j} J_{i j} \hat{S}_{i} \cdot \hat{S}_{j}
$$

onde $\hat{S}_{i}$ é o vetor unitário indicando a direção e o sentido do momento magnético de spin no sítio $i$, e o termo $J_{i j}$, chamado de interação de troca, descreve a interação magnética entre os sítios $i$ e $j$. Para $J_{i j}>0$ a configuração FM é estável e para $J_{i j}<0$, o estado fundamental é AF. O modelo de Heisenberg é usualmente adequado para tratar sistemas onde os momentos magnéticos são localizados. Neste caso, são freqüentemente consideradas somente as interações entre primeiros vizinhos (considerando-se $J_{i j}=0$ para vizinhos mais distantes). Para flutuações de spin em sistemas ferromagnéticos de elétrons itinerantes, o modelo de Heisenberg também pode ser aplicado sob certas condições. Entretanto, o alcance para o qual $J_{i j}$ é diferente de zero deve ser aumentado, levando em conta interações além das 
de primeiros vizinhos. O modelo de Heisenberg pode ser utilizado para estudar configurações do estado fundamental, assim como o comportamento magnético a temperaturas finitas [77]. Neste trabalho, nossos resultados $a b$ initio para o estado fundamental são discutidos no contexto do modelo de Heisenberg. Uma situação interessante é quando dois átomos magnéticos são separados por átomos não magnéticos. Neste caso, os elétrons de condução são fundamentais para compreendermos o surgimento da ordem magnética. Por exemplo, um átomo magnético localizado em uma posição $R$ interage, via interação de troca, com um elétron de condução. Tal interação transmite ao elétron a informação sobre o estado de polarização do átomo. Ao se propagar pelo cristal e interagir com outro átomo em uma posição $R^{\prime}$, este tenderá a se alinhar de acordo com a polarização do elétron [78]. Estas interações são descritas pela teoria RKKY [79-81] e relacionam as interações entre átomos isolados e também entre camadas magnéticas separadas por um espaçador não magnético. Nossos resultados para a descrição das interações de troca magnética entre os átomos de uma nanoestrutura de Mn em Fe(001) e Fe(110) são comparados ao comportamento previsto pela teoria RKKY.

\subsubsection{Interação de Dzyaloshinskii-Moriya}

Na seção anterior apresentamos o modelo de Heisenberg [Eq.(3.1)] para o cálculo da interação de troca magnética entre os momentos magnéticos localizados nos sítios $i$ e $j$. Uma importante propriedade da interação de troca magnética é ser isotrópica devido à interação Coulombiana. Desta forma, as configurações magnéticas $(\uparrow \downarrow)$ e $(\downarrow \uparrow)$ têm a mesma energia. Devemos também levar em consideração que as nanostruturas metálicas não possuem a simetria de inversão, devido à presença de interfaces, defeitos e superfícies. Este fato implica que a interação de spin-órbita fornece uma interação adicional na Hamiltoniana de Heisenberg [Eq.(3.1)]. Este termo recebe o nome de interação de Dzyaloshinskii-Moriya (DM) [82,83]:

$$
E_{D M}=\sum_{i, j} \vec{D}_{i j} \cdot\left(\hat{S}_{i} \times \hat{S}_{j}\right)
$$


onde $\vec{D}_{i j}$ é chamado de vetor de Dzyaloshinskii. Quando esta interação for importante, a sequência de espalhamento dos elétrons de spin polarizados não é comutativa, por exemplo: $i \rightarrow j \rightarrow i$ é diferente de $j \rightarrow i \rightarrow j$. A presença da interação DM tem como consequência estruturas magnéticas complexas, como por exemplo, estruturas de spin espirais [3,4] e também estruturas do tipo skyrmions $[5,6]$. Dentro deste contexto, as redes de skyrmions podem ficar estabilizadas quando não existe uma simetria de translação e, desta forma, as interações de DM podem ser importantes. As redes de skyrmions (Fig. 3.2) podem ser vistas como momentos magnéticos giratórios parecidos com um redemoinho (vórtice magnético). Geralmente tinha-se assumido que skyrmions não poderiam formar estados fundamentais espontâneos, tais como FM e AF, em materiais magnéticos. Entretanto, foi mostrado por U. K. Rößler et al. [84] a possibilidade da existência de configurações tipo skyrmions em materiais magnéticos devido a presença de interações quirais, sem a necessidade de campos externos. Isto pode ser observado em uma monocamada de Fe em $\operatorname{Ir}(111)$ [6].

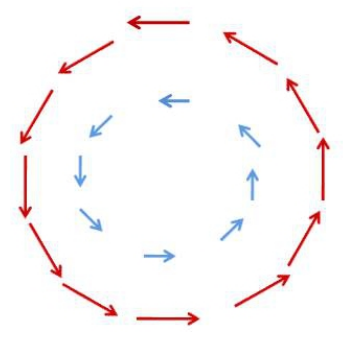

Figura 3.2: Ilustração de uma rede de skyrmions que pode ser vista como um redemoinho de momentos magnéticos.

\subsection{Cálculo da interação de troca com o RS- LMTO-ASA}

A interação de troca magnética pode ser obtida através da equação de Liechtenstein $[85,86]$, onde as mudanças de energias relacionadas as rotações 
infinitesimais dos momentos dos sítios $i$ e $j$ são calculadas utilizando o Teorema da Força. Dentro do método RS-LMTO-ASA, o cálculo da interação de troca magnética segue uma abordagem bem similar [87], onde a interação de troca magnética é calculada como:

$$
J_{i j}=\frac{1}{4 \pi} \operatorname{Im} \operatorname{Tr} \int_{-\infty}^{E_{F}} d E\left[\delta_{i}(E) G_{i j}^{\Uparrow}(E) \delta_{j}(E) G_{j i}^{\downarrow}(E)\right] .
$$

Aqui $\Im T r$ denota a parte imaginária do traço da matriz dentro dos colchetes, a função de Green $G_{i j}^{\sigma \sigma}$ tem o inverso da energia e é o propagador para os elétrons com spin $\sigma$ entre os sítios $i$ e $j$, e $\delta_{i}$ é uma matriz diagonal dentro do conjunto dos orbitais ( $s, p$ e $d$ ), que pode ser escrita como:

$$
\delta_{l i}=\frac{C_{l i}^{\downarrow} \Delta_{l i}^{\uparrow}-C_{l i}^{\uparrow} \Delta_{l i}^{\downarrow}+\left(\Delta_{l i}^{\downarrow}-\Delta_{l i}^{\uparrow}\right) E}{\left(\Delta_{l i}^{\downarrow} \Delta_{l i}^{\uparrow}\right)^{1 / 2}},
$$

onde $l$ são os índices orbitais $(l=0,1$ e 2$)$ associados com elétrons das bandas $s, p$ e $d, C_{l i}^{\sigma}$ e $\Delta_{l i}^{\sigma}$ são parâmetros de potencial do sítio $i$ na representação ortogonal. A quantidade $\delta_{i}(E)$ é associada com o exchange splitting local

no sítio $i$. Quando $\Delta_{i}^{\downarrow}=\Delta_{i}^{\uparrow}$, a Eq.(3.4) se reduz a uma diferença entre os centros das bandas de energia dos spin up e down, condição que é usada freqüentemente nas parametrizações TB.

Neste trabalho, verificamos as estabilidades das configurações magnéticas estudadas através dos cálculos de $J_{i j}$ entre os sítios em cada estrutura estudada. Por exemplo, começamos o cálculo com uma configuração FM: caso o valor da interação de troca seja positivo, esta configuração é estável; por outro lado, um valor negativo mostra que o estado FM é instável. Este mesmo procedimento pode ser usado para a configuração AF. Além disto, podem existir casos em que os valores de $J_{i j}$ sejam negativos tanto nas configurações FM quanto AF, o que indica que o ordenamento não colinear pode ser o fundamental [43].

\subsection{Ocorrência do magnetismo não colinear}

Dentre as diferentes situações que levam a um ordenamento magnético não colinear, destacamos aqui, no caso de sistemas constituídos por na- 
noestruturas adsorvidas em superfícies metálicas, as frustrações geométricas [88] e competições entre as interações de troca magnética [89].

\subsubsection{Frustração}

O termo frustração magnética refere-se à inabilidade de que átomos vizinhos satisfaçam às interações de troca. Esta situação é ilustrada na [Fig. 3.3], onde consideramos um triângulo eqüilátero com os átomos ocupando seus vértices: a configuração FM é ilustrada na [Fig. 3.3-(a)] e vemos na [Fig. 3.3(b)] que uma configuração onde todos os pares satisfaçam um acoplamento AF é impossível devido a geometria . Assim, usando a Hamiltoniana de Heisenberg, pode-se mostrar que o estado de menor energia é tal que os momentos magnéticos de spin apresentam um alinhamento de $120^{\circ}$ entre eles, levando a um estado fundamental não colinear como mostrado na [Fig.3.3(c)].

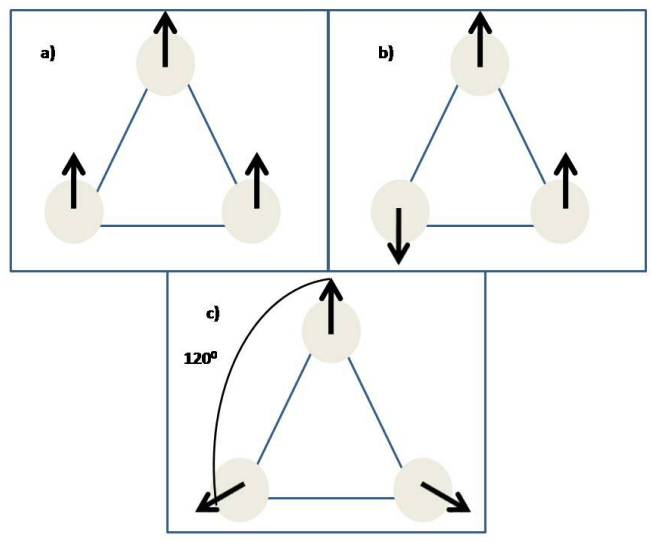

Figura 3.3: Ilustração de átomos magnéticos colocados em um triângulo eqüilátero: (a) solução FM, (b) solução AF frustrada e (c) solução não colinear onde os momentos fazem um ângulo de $120^{\circ}$ com os vizinhos. 


\subsubsection{Competição entre interações de troca FM e AF}

Na Fig. 3.4 mostramos a situação onde pode ocorrer competição entre as interações FM e AF entre átomos constituintes da nanoestrutura e os átomos do substrato. Para exemplificar este caso consideremos um dímero com um acoplamento AF [Fig. 3.4-(a)] e um substrato FM [Fig. 3.4-(b)], onde o acoplamento entre cada átomo do dímero com os do substrato também é FM e de mesma ordem de grandeza [Fig. 3.4-(c)]. Esta competição entre as interações de troca magnética leva o dímero a uma configuração magnética não colinear [Fig. 3.4-(d)], conforme descrito anteriormente.

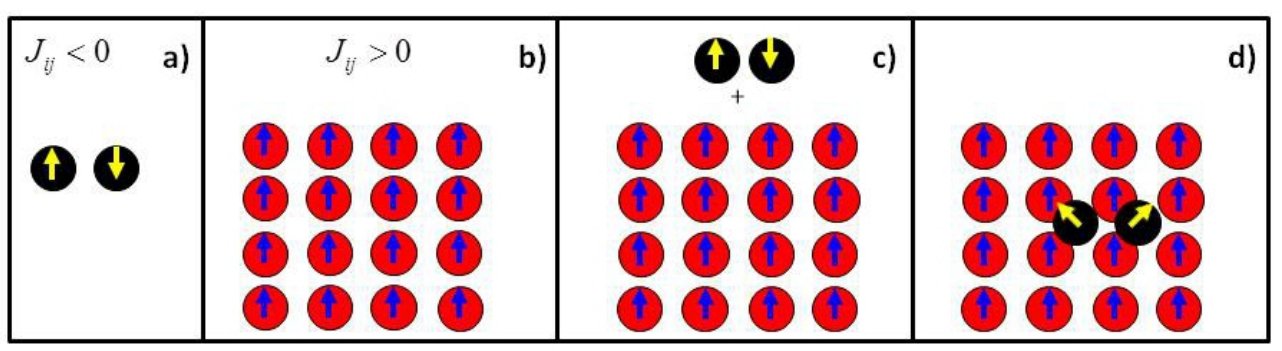

Figura 3.4: Esquema mostrando a competição da interação de troca FM e AF entre os momentos magnéticos dos átomos da nanoestrutura e do substrato: (a) dímero AF, (b) substrato FM, (c) dímero depositado em um substrato FM e (d) devido a competição entre as interações de troca ocorre uma estrutura magnética não colinear no dímero.

\subsection{Cálculo do magnetismo não colinear}

Na literatura existem diferentes formalismos que são capazes de tratar o magnetismo não colinear de átomos livres e sistemas periódicos [90-92]. Contudo, poucos métodos são capazes de descrever o ordenamento magnético não colinear de aglomerados de átomos adsorvidos em superfícies. Há diferentes abordagens para o magnetismo não colinear. Uma abordagem possível é permitir a não colinearidade intra-atômica. Outra permite apenas a magnetização não colinear inter-atômica, onde cada átomo possui um único eixo de 
quantização de spin. Dentro do RS-LMTO-ASA, utilizamos a magnetização inter-atômica [40,41].

A densidade de estados local, $N(E)$, pode ser calculada através do método de Recorrência (Apêndice B), a partir da seguinte relação

$$
N(E)=-\frac{1}{\pi} \operatorname{Im} \operatorname{Tr}[G(E)]
$$

onde $G(E)$ é a função de Green local dada por:

$$
G(E)=(E-H)^{-1}
$$

Analogamente ao processo para obter a $N(E)$ a densidade magnética, $m(E)$, pode ser expressa como:

$$
m(E)=-\frac{1}{\pi} \operatorname{Im} \operatorname{Tr}[\sigma G(E)] .
$$

No caso do magnetismo colinear, existe apenas um único eixo global de magnetização (eixo $z$ ) e a densidade de estados magnéticos pode ser calculada como:

$$
m_{z}(E)=-\frac{1}{\pi} \operatorname{Im} \operatorname{Tr}\left[\sigma_{z} G(E)\right]
$$

onde são usados os elementos diagonais da função de Green. No cálculo da densidade de magnetização não colinear [Eq. 3.7] é necessário, a priori, calcular os termos não diagonais da função de Green que podem ser calculados por vários procedimentos na literatura [43,93,94]. Entretanto, o cálculo da parte não diagonal da função de Green é computacionalmente custoso.

Para evitar os cálculos dos termos fora da diagonal são aplicadas sucessivas transformações unitárias $U$ sobre a Hamiltoniana. Desde que $\sigma_{z}$ é diagonal, com a Eq.(3.8) $m_{z}(E)$ pode ser obtido usando os elementos diagonais da função de Green. Para as outras componentes de $\sigma$ efetua-se rotações de $\sigma$ para $\sigma^{\prime}$, de tal forma que $\sigma_{x}^{\prime}$ seja uma matriz diagonal. Desta forma, obtemos $m_{x}(E)$ e, analogamente, realizando uma rotação para obter $\sigma_{y}^{\prime}$ diagonal, obtemos $m_{y}(E)$, o que nos possibilita construir $m(E)$. O problema é como realizar essas rotações?

As rotações podem ser feitas da seguinte forma: representamos a rotação como uma transformação unitária, $U$. Aplicando esta transformação na 
Hamiltoniana, $H^{\prime}=U H U^{\dagger}$, implica também que a função de Green tenha a mesma transformação, $G^{\prime}=U G U^{\dagger}$. Usando a propriedade $U^{\dagger} U=1 \mathrm{e}$ que o traço é um invariante sobre rotações, podemos escrever a densidade de estados magnéticos da seguinte forma:

$$
m(E)=-\frac{1}{\pi} \Im \operatorname{Tr}\left\{\sigma U^{\dagger} U G U^{\dagger} U\right\}=-\frac{1}{\pi} \Im \operatorname{Tr}\left[\sigma^{\prime} G^{\prime}\right],
$$

onde $\sigma^{\prime}$ são as matrizes de spin de Pauli após as transformações unitárias. Escolhendo as matrizes unitárias $U_{1}$ e $U_{2}$ de tal forma que $\sigma_{x}^{\prime}$ e $\sigma_{y}^{\prime}$ são matrizes diagonais, podemos calcular $m_{x}(E)$ e $m_{y}(E)$ através dos elementos diagonais da função de Green. Estas transformações correspondem as seguintes rotações de spin

$$
\sigma_{x}^{\prime}=U_{1} \sigma_{x} U_{1}^{\dagger}
$$

e

$$
\sigma_{y}^{\prime}=U_{1} \sigma_{y} U_{1}^{\dagger}
$$

Também podemos definir uma transformação unitária $U_{3}$ onde esta transformação é justamente a matriz identidade, a qual torna $\sigma_{z^{\prime}}$ diagonal.

A expressão (2.8) mostra que a Hamiltoniana pode ser separada em uma parte independente de spin $\left(H^{0}\right)$ e uma parte dependente de spin

$$
H=H^{0} \mathbf{1}+\mathbf{b} \cdot \sigma
$$

Se aplicarmos uma transformação unitária na Hamiltoniana, somente a parte dependente de spin vai ser transformada

$$
H^{\prime}=H^{0} \mathbf{1}+\mathbf{b} \cdot U \sigma U^{\dagger}
$$

Representamos os sítios $R$ e orbitais $L$ dos elementos de matriz como $Q=$ $R L$ e os parâmetros de potencial das partes independentes de spin com o superscrito 0 e das partes dependentes de spin com o superscrito 1. Os elementos de matriz da parte independente de spin $H^{0}$ da Hamiltoniana tem a seguinte forma:

$$
H_{Q Q^{\prime}}^{0}=\bar{C}_{Q}^{0}+{\overline{\Delta_{Q}^{0}}}^{1 / 2} S_{Q Q^{\prime}}^{-} \bar{\Delta}_{Q^{\prime}}^{0}{ }^{1 / 2}+\bar{\Delta}_{Q}^{\bar{C}^{1 / 2}} S_{Q Q^{\prime}}^{-} \bar{\Delta}_{Q^{\prime}}^{1}{ }^{1 / 2} \mathbf{m}_{Q} \cdot \mathbf{m}_{Q^{\prime}}
$$


a parte dependente de spin é escrita como

$$
\begin{aligned}
\mathbf{b}= & \left(\bar{C}_{Q}^{1}+{\overline{\Delta_{Q}^{1}}}^{1 / 2} S_{Q Q^{\prime}}^{-}{\overline{\Delta_{Q}^{0}}}^{1 / 2}\right) \mathbf{m}_{Q}+{\overline{\Delta_{Q}^{0}}}^{1 / 2} S_{Q Q^{\prime}}^{-}{\overline{\Delta_{Q^{\prime}}^{0}}}^{1 / 2} \mathbf{m}_{Q^{\prime}} \\
& +\bar{\Delta}_{Q}^{{ }^{1}}{ }^{1 / 2} S_{Q Q^{\prime}}^{-} \bar{\Delta}_{Q^{\prime}} \overline{1}^{1 / 2} \mathbf{m}_{Q} \times \mathbf{m}_{Q^{\prime}}
\end{aligned}
$$

Aqui m representa as componentes do momento magnético e com a Hamiltoniana usamos o método de Recorrência, três vezes consecutivas, para as transformações unitárias $U_{1}, U_{2}$ e $U_{3}$, obtendo $m_{x}(E), m_{y}(E)$ e $m_{z}(E)$. A direção da magnetização local pode ser obtida, integrando estas componentes da densidade de magnetização até a energia de Fermi. 


\section{Capítulo 4}

\section{Propriedades Magnéticas de Nanoestruturas de Mn Sobre a Superfície Fe(001)}

Neste capítulo, apresentamos os resultados obtidos usando o RS-LMTOASA para estrutura eletrônica e propriedades magnéticas de nanoestruturas de Mn em uma superfície Fe(001) cúbica de corpo centrado (bcc). Consideramos uma variedade de nanoestruturas tais como fios adsorvidos, pirâmides, aglomerados adsorvidos e também embebidos na primeira camada de Fe(001).

\subsection{Preâmbulo}

Como dissemos no Capítulo 1 um completo entendimento microscópico do mecanismo que ocorre nas interfaces é ainda um desafio devido à presença de vários tipos de ordenamentos químicos e magnéticos. Neste contexto, camadas de Mn na superfície Fe(001) constituem um sistema interessante para a investigação do acoplamento magnético através de uma interface ferromagneto-antiferromagneto devido a intricada dependência entre magnetismo e a espessura dos filmes. Resultados experimentais indicam que filmes de Mn com duas ou mais camadas atômicas têm os momentos magné- 
ticos acoplados AF entre as camadas de Mn, mas FM acoplados com o momento magnético da camada de Fe na interface entre Mn e o Fe [19,95-98]. Estes filmes finos de Mn (até duas camadas) em Fe(001) ficam estabilizados em uma estrutura tetragonal centrada (bct), próximo da estrutura Fe bcc [99-102]. Aumentando a quantidade de camadas de Mn a distância entre os planos atômicos de Mn aumenta $[100,103]$. Entretanto, para uma única cobertura de Mn, a camada de Mn tem uma estrutura AF não colinear no plano com os momentos magnéticos do Mn aproximadamente perpendiculares à magnetização do Fe [16]. Este resultado está de acordo com os pequenos valores observados experimentalmente para a magnetização da camada de Mn [16-18]. Por outro lado, para sub-monocamadas de Mn em $\mathrm{Fe}(001)$, estudos experimentais mostram que os momentos magnéticos do Mn acoplam-se ferromagneticamente entre eles, mas são antiparalelos a magnetização do Fe [17-19]. Para filmes de Mn crescidos em uma superfície Fe(001) em um degrau monoatômico, um ordenamento magnético não colinear aparece nas vizinhanças do defeito [20,96, 104, 105], entretanto, a presença de interdifusão de átomos de Mn incorporados na camada de Fe pode deixar o problema ainda mais complicado [17,103,106,107]. Estudos teóricos para a estrutura magnética de filmes finos de Mn adsorvidos em $\mathrm{Fe}(001)$ têm muito interesse atual, como pode ser visto nas Refs. [16,20-29]. Contudo, existem somente alguns estudos para aglomerados de Mn em uma superfície $\mathrm{Fe}(001)$ bcc $[22,30]$. Desta forma, investigamos a estrutura eletrônica e propriedades magnéticas das nanoestruturas de Mn em Fe(001).

\subsection{Detalhes computacionais}

Para a superfície $\mathrm{Fe}(001)$ utilizamos um cluster contendo 10560 átomos posicionados em uma rede $b c c$ com o parâmetro de rede do Fe $(2,87 \AA)$. No Método de Recorrência, a fração continuada foi calculada incluindo até 20 níveis de recorrência e utilizando o terminador de Beer-Pettifor [68] (Apêndice B). Foram colocadas duas camadas de esferas vazias acima da superfície Fe, para simular o vácuo e incluir as transferências de cargas corretamente. Os 
cálculos das nanoestruturas de Mn foram feitos depositando os aglomerados como uma perturbação da superfície $\mathrm{Fe}(001)$ autoconsistente, isto é, onde os parâmetros de potencial para os outros sítios distantes do aglomerado de Mn foram mantidos constantes. Os sítios de Mn assim como os primeiros e segundos vizinhos Fe (incluídos também as esferas vazias) dos sítios de Mn, foram recalculados autoconsistentemente, com o tamanho desta região variando entre 10 até 78 sítios.

Também fizemos cálculos com e sem relaxação estrutural. Neste último, consideramos que os átomos de Mn ocupam posições não relaxadas (ocupados por esferas vazias), assumindo o parâmetro de rede do substrato Fe. Nos cálculos com relaxação, consideramos relaxações perpendiculares afastando e aproximando os átomos de Mn da superfície Fe(001) entre $2 \%$ e 6\%. Estas escolhas são baseadas em resultados experimentais, onde foi observado que a monocamada de Mn depositada em $\mathrm{Fe}(001)$ cresce em uma estrutura bct, com um parâmetro de rede do Fe, no plano, de $2,87 \AA$ e uma distância fora do plano, $d_{\perp}$, que pode variar com a espessura do filme de Mn. Por exemplo, nos filmes ultrafinos com até duas camadas de $\mathrm{Mn}$ em $\mathrm{Fe}(001)$, a distância interplanar é $d_{\perp}^{F e M n} \approx 1,4 \AA$, onde a separação entre as camadas de Mn é $d_{\perp}^{M n M n} \approx$

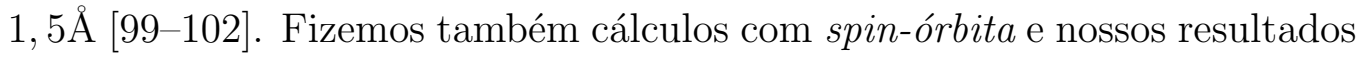
indicam que os momentos magnéticos orbitais são relativamente pequenos, em torno de 0,04 $\mu_{B}$ /átomo e 0,1 $\mu_{B}$ /átomo para os átomos de Mn e Fe, respectivamente. Para as configurações magnéticas não colineares, também fizemos cálculos com acoplamento spin-órbita. Dentro deste contexto, para um nanofio formado por nove átomos de Mn, também fizemos cálculos sem o acoplamento spin-órbita, para entender os efeitos desta interação sobre a configuração magnética do sistema.

As interações de troca de Heisenberg $J_{i j}$ foram calculadas através da fórmula de Liechtenstein et al. [85], como implementado no RS-LMTO-ASA [Eq. 3.3]. Os valores obtidos de $J_{i j}$ são usados para analisar, em nível quantitativo, a competição da interação de troca magnética entre os vizinhos do Mn, bem como efeitos de frustração do ordenamento magnético, analisados em todos os cálculos não colineares. 


\subsection{Estrutura eletrônica do Fe bcc bulk e da superfície $\mathrm{Fe}(001)$}

\subsubsection{Fe bulk}

Para simular a estrutura cristalina no cálculo da estrutura eletrônica do Fe bulk, usamos uma rede cúbica de corpo centrado bcc com 4957 átomos e parâmetro de rede experimental $a=2,87 \AA$. Os resultados das ocupações por orbital das bandas majoritárias $(\uparrow)$ e minoritárias $(\downarrow)$ de um átomo de Fe ocupando uma posição aproximadamente central do aglomerado estão mostrados na Tabela 4.1. Vale ressaltar que nos nossos cálculos dentro da DFT, fica difícil estimar uma incerteza nos resultados, devido às diversas aproximações utilizadas. Contudo, estimamos incertezas da ordem de $10^{-2}$ $\mu_{B}$ no momento magnético, $10^{-1} \mathrm{meV}$ nas interações de troca magnética e $10^{-3}$ elétrons nas transferências de cargas.

Tabela 4.1: Ocupações por orbital para as bandas majoritárias (spin $\uparrow$ ) e minoritárias (spin $\downarrow$ ) do Fe bulk bcc.

\begin{tabular}{|c|c|c|}
\hline & spin $\uparrow$ & spin \\
\hline $\mathrm{s}$ & 0,334 & 0,356 \\
\hline $\mathrm{p}$ & 0,368 & 0,443 \\
\hline $\mathrm{d}$ & 4,352 & 2,148 \\
\hline
\end{tabular}

Na Fig. 4.1 apresentamos a correspondente densidade de estados local. Como já reportado na literatura, a banda majoritária da DOS para o Fe bulk $b c c$ apresenta esta estrutura de três picos que, por sua vez, é uma característica geral dos metais de transição de estruturas bcc (Mo, Nb, V e Cr) [108]. O momento magnético de spin (diferença entre as ocupações das bandas majoritárias e minoritárias) de 2,11 $\mu_{B}$, aqui obtido, está em boa concordância com outros resultados teóricos da literatura [108] e com o valor experimental $2,13 \mu_{B}[109]$. 


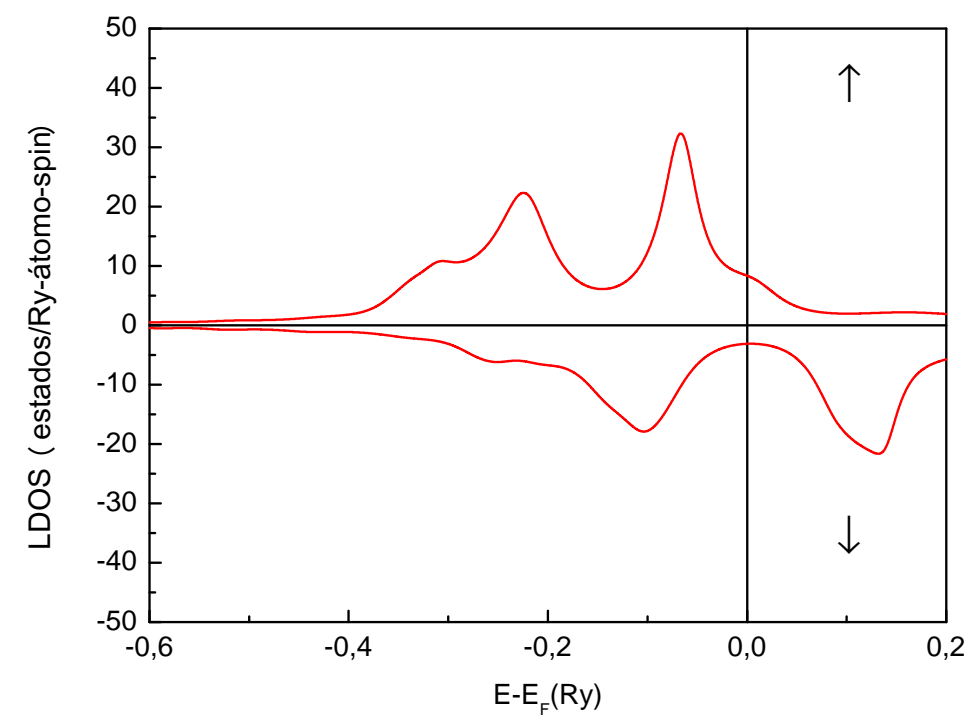

Figura 4.1: Densidade de estados local (LDOS) do Fe metálico. A linha vertical passando por zero refere-se ao nível de Fermi.

\subsubsection{Superfície $\mathrm{Fe}(001)$}

Para simular a superfície Fe(001), partimos da rede cúbica de corpo centrado do Fe bulk com parâmetro de rede experimental $(a=2,87 \AA)$ e construímos um aglomerado com uma simetria tal que em cada plano (xy) com $z=D a$ (onde $\mathrm{D}$ é igual a uma constante e $a$ denota o parâmetro de rede) e no plano adjacente a este, caracterizado por ter $z=(D+0,5) a$, todos os átomos são equivalentes entre si, isto é, possuem as mesmas LDOS. Consideramos o sistema contendo 18 camadas de Fe, além de justaposto ao plano da superfície, dois planos compostos por esferas vazias, colocadas para simular o vácuo: em $z=-1,0 a$ (denotado por ESF-1) e $z=-0,5 a$ (denotado por ESF-2). Denotamos por Fe(S), Fe(S-1), Fe(S-2), etc o plano de átomos de Fe da superfície em $z=0$ e para os planos de Fe localizados em $z=0,5 a$, $z=1,0 a$ e assim sucessivamente. Uma representação esquemática desta superfície é mostrada na Fig. 4.2. O critério usado na escolha do número de camadas de Fe incluídas de forma autoconsistente nos cálculos foi o de 
que a camada mais interna tivesse características (i.e. ocupação, LDOS e parâmetros de potencial) próximas das do Fe bulk, dentro de um critério de convergência pré-estabelecido. Consideramos assim, para o cálculo da superfície $\mathrm{Fe}(001)$ incluídas de forma autoconsistente, duas camadas de esferas vazias (ESF-1 e ESF-2) e seis camadas de Fe [Fe(S), Fe(S-1), Fe(S-2), Fe(S-3), $\mathrm{Fe}(\mathrm{S}-4)$ e $\mathrm{Fe}(\mathrm{S}-5)]$, sendo que para as demais camadas de $\mathrm{Fe}$, os parâmetros de potencial foram fixados naqueles obtidos para o Fe bulk.

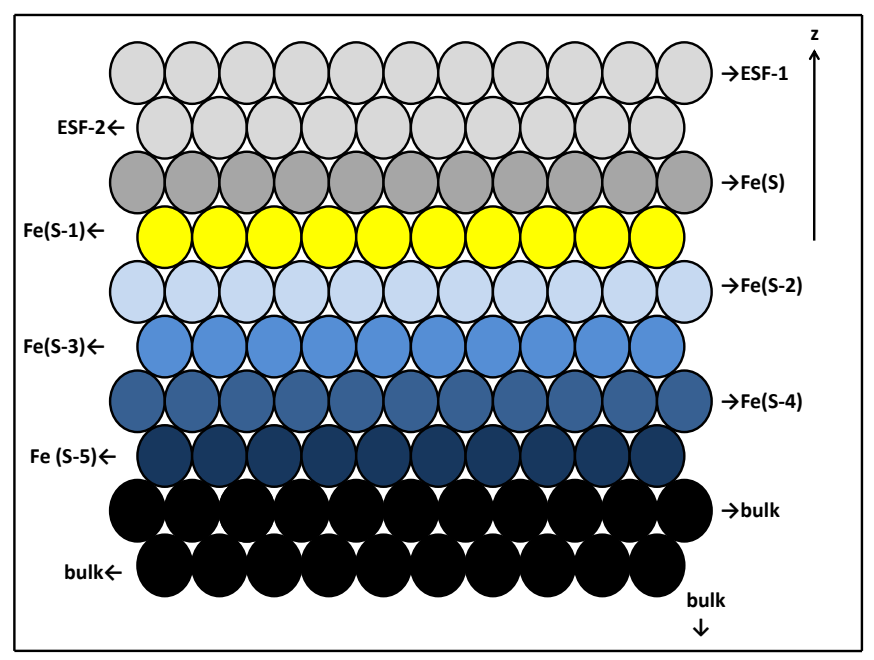

Figura 4.2: Representação esquemática para o arranjo atômico de uma superfície de $\mathrm{Fe}(001)$.

Na Tabela 4.2, mostramos os resultados obtidos de forma autoconsistente para as ocupações de cada camada de Fe. Podemos verificar que a partir da quarta camada $[\mathrm{Fe}(\mathrm{S}-3)]$ os valores das ocupações são próximos aos obtidos para o Fe bulk.

Na Tabela 4.3 apresentamos nossos resultados para os momentos magnéticos locais $\left(\operatorname{spin} m_{s}\right.$ e orbital $m_{L}$ ) e transferências de carga para as diversas camadas. Definimos aqui uma transferência de carga positiva quando o sítio ganha elétrons, enquanto que uma transferência de carga negativa indica que elétrons deixaram o sítio. Verificamos que alguns elétrons deixam a camada superficial $\mathrm{Fe}(\mathrm{S})$, que apresenta uma transferência de carga negativa. Observamos também um efeito de blindagem das cargas da superfície, onde as 
cargas da camada $\mathrm{Fe}(\mathrm{S})$ são transferidas para as duas camadas adjacentes. Com relação aos $m_{L}$, obtivemos que estes são pequenos, comparados aos $m_{s}$. Enquanto, existe um aumento do $m_{s}$ dos átomos de Fe localizados na camada da superfície $[\mathrm{Fe}(\mathrm{S})]$, se comparado ao momento magnético do Fe bulk. Isto pode ser explicado devido ao fato que os átomos da camada $\mathrm{Fe}(\mathrm{S})$ apresentam um menor número de coordenação. Verificamos também que há uma oscilação nos $m_{s}$ nas camadas adjacentes à superfície e os átomos das camadas mais internas tendem ao $m_{s}$ para o Fe bulk bcc. Estes resultados estão em boa concordância com os obtidos experimentalmente [110] e por outros métodos ab initio baseados na DFT [111].

Na Fig. 4.3 são apresentadas as LDOS para as diferentes camadas de Fe. Podemos observar que a LDOS para o Fe na camada da superfície $(\mathrm{Fe}(\mathrm{S}))$ é bastante modificada se comparada com a LDOS do Fe bulk (Fig. 4.1), devido, como é sabido na literatura [112], à diminuição do número de primeiros vizinhos dos átomos na camada da superfície. Notamos que a banda majoritária da camada $\mathrm{Fe}(\mathrm{S})$ apresenta-se quase completamente cheia e a banda minoritária da LDOS é deslocada para energias maiores, apresentando um

Tabela 4.2: Ocupações por orbital para as bandas majoritárias ( $\uparrow$ ) e minoritárias $(\downarrow)$ das diferentes camadas consideradas para o cálculo da superfície livre $\mathrm{Fe}(001)$.

\begin{tabular}{|c|c|c|c|c|c|c|}
\hline camadas & $\mathrm{s} \uparrow$ & $\mathrm{p} \uparrow$ & $\mathrm{d} \uparrow$ & $\mathrm{s} \downarrow$ & $\mathrm{p} \downarrow$ & $\mathrm{d} \downarrow$ \\
\hline \hline ESF-1 & 0,005 & 0,005 & 0,002 & 0,002 & 0,002 & 0,001 \\
\hline ESF-2 & 0,091 & 0,071 & 0,032 & 0,074 & 0,049 & 0,019 \\
\hline $\mathrm{Fe}(\mathrm{S})$ & 0,308 & 0,231 & 4,696 & 0,301 & 0,219 & 1,742 \\
\hline $\mathrm{Fe}(\mathrm{S}-1)$ & 0,342 & 0,378 & 4,369 & 0,369 & 0,449 & 2,244 \\
\hline $\mathrm{Fe}(\mathrm{S}-2)$ & 0,338 & 0,376 & 4,386 & 0,356 & 0,444 & 2,104 \\
\hline $\mathrm{Fe}(\mathrm{S}-3)$ & 0,333 & 0,367 & 4,355 & 0,355 & 0,440 & 2,144 \\
\hline $\mathrm{Fe}(\mathrm{S}-4)$ & 0,335 & 0,367 & 4,368 & 0,356 & 0,442 & 2,131 \\
\hline $\mathrm{Fe}(\mathrm{S}-5)$ & 0,334 & 0,368 & 4,347 & 0,356 & 0,442 & 2,153 \\
\hline $\mathrm{Fe}$ bulk & 0,334 & 0,368 & 4,351 & 0,356 & 0,443 & 2,147 \\
\hline
\end{tabular}


Tabela 4.3: Momento magnético ( $\operatorname{spin} \mathrm{m}_{s}$ e orbital $\mathrm{m}_{L}$ ) em unidades de $\mu_{B}$ e transferências de carga $\Delta Q$ (em unidades de elétron) para as várias camadas de Fe (001).

\begin{tabular}{|c|c|c|c|}
\hline camadas & $m_{s}$ & $m_{L}$ & $\Delta Q$ \\
\hline \hline ESF-1 & 0,00 & 0,00 & 0,01 \\
\hline ESF-2 & 0,05 & 0,00 & 0,34 \\
\hline $\mathrm{Fe}(\mathrm{S})$ & 2,97 & 0,10 & $-0,50$ \\
\hline $\mathrm{Fe}(\mathrm{S}-1)$ & 2,03 & 0,04 & 0,15 \\
\hline $\mathrm{Fe}(\mathrm{S}-2)$ & 2,19 & 0,04 & 0,00 \\
\hline $\mathrm{Fe}(\mathrm{S}-3)$ & 2,12 & 0,04 & 0,00 \\
\hline $\mathrm{Fe}(\mathrm{S}-4)$ & 2,14 & 0,04 & 0,00 \\
\hline $\mathrm{Fe}(\mathrm{S}-5)$ & 2,10 & 0,04 & 0,00 \\
\hline $\mathrm{Fe}$ bulk & 2,11 & 0,04 & 0,00 \\
\hline
\end{tabular}

menor número de estados ocupados quando comparada à banda minoritária da LDOS do Fe bulk. Como conseqüência, há um aumento na diferença entre as ocupações das bandas majoritárias e minoritárias, correspondendo a um aumento no momento magnético da camada da superfície em relação ao Fe bulk. A perturbação introduzida pela superfície tende a decair para as camadas mais internas, onde a LDOS da camada $\mathrm{Fe}(\mathrm{S}-2)$ apresenta uma forma próxima à da LDOS Fe bulk. 

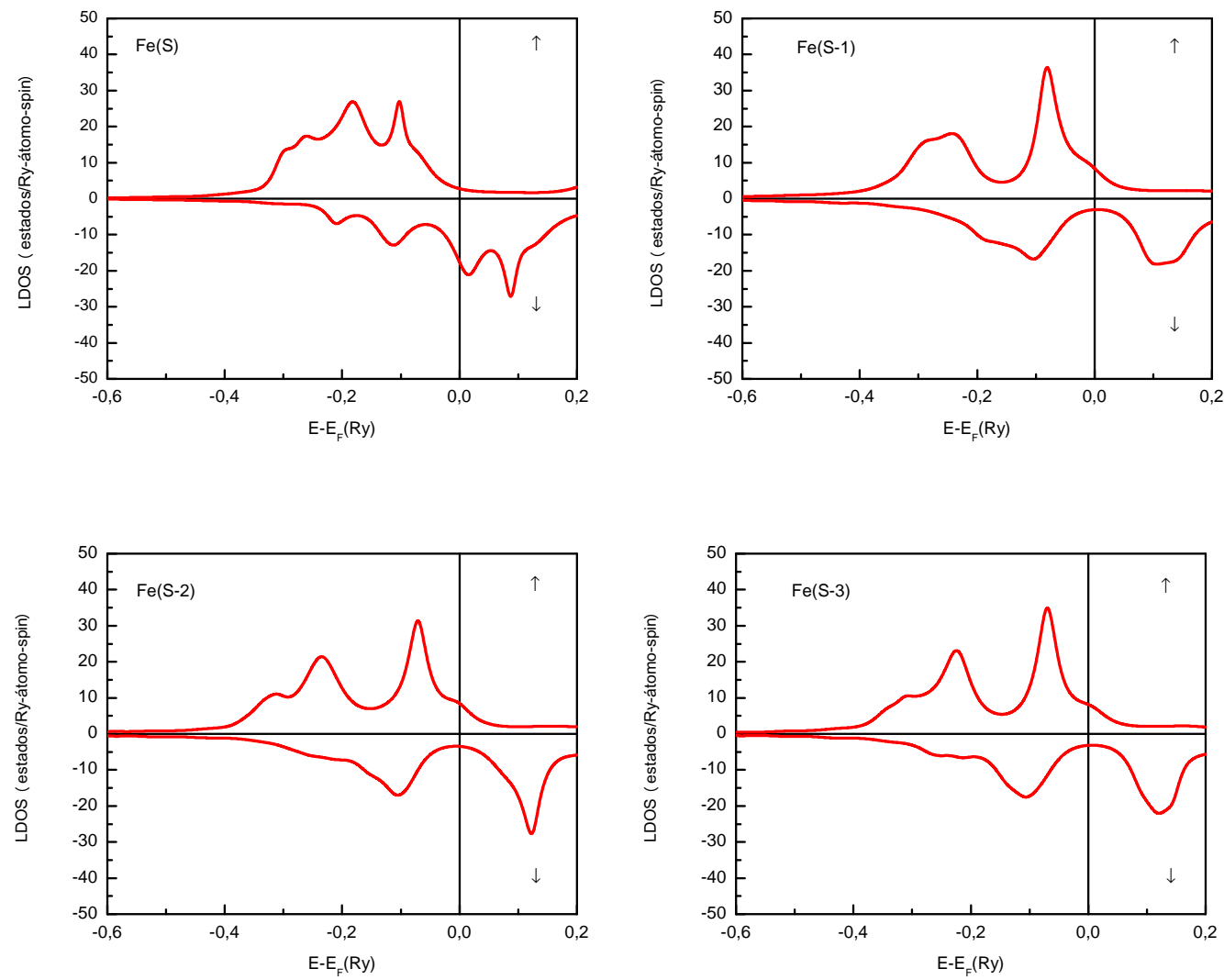

Figura 4.3: Densidade de estados local para os átomos de Fe nas camadas $\mathrm{Fe}(\mathrm{S}), \mathrm{Fe}(\mathrm{S}-1), \mathrm{Fe}(\mathrm{S}-2)$ e $\mathrm{Fe}(\mathrm{S}-3)$ da superfície $\mathrm{Fe}(001)$.

\subsection{Sistemas unidimensionais de Mn sobre a superfície de $\mathrm{Fe}(001)$}

\subsubsection{Adátomo de Mn sobre a superfície Fe(001)}

Estudamos inicialmente as propriedades magnéticas de um adátomo Mn sobre a superfície Fe(001). Utilizamos o método RS-LMTO-ASA para defeitos em superfícies, conforme apresentado no Capítulo 2, usando o mesmo aglomerado que no cálculo da superfície livre de Fe(001) descrito na seção anterior, exceto pela substituição de um sítio de esfera vazia por um átomo de Mn, numa posição quase central. 
Objetivando determinar qual é o número suficiente de camadas de vizinhos em torno do defeito que descreve corretamente o Mn sobre a superfície $\mathrm{Fe}(001)$, realizamos inicialmente cálculos autoconsistentes incluindo primeiros e segundos vizinhos em torno do adátomo em Fe(001) (Fig. 4.4). Conforme mencionamos anteriormente, nos cálculos autoconsistentes com primeiros vizinhos, os parâmetros de potencial da primeira camada de Fe e de esferas vazias, em torno do defeito, são calculados de forma autoconsistente, enquanto que os parâmetros de potencial de todos os outros sítios são fixados nos valores obtidos para a superfície livre. Analogamente, cálculos autoconsistentes com até segundos vizinhos indicam que os parâmetros de potencial das duas primeiras camadas de átomos de Fe e esferas vazias, em torno do defeito, são calculados de forma autoconsistente, enquanto que os parâmetros de potencial de todos os outros sítios são fixados nos valores para a superfície livre $\mathrm{Fe}(001)$.

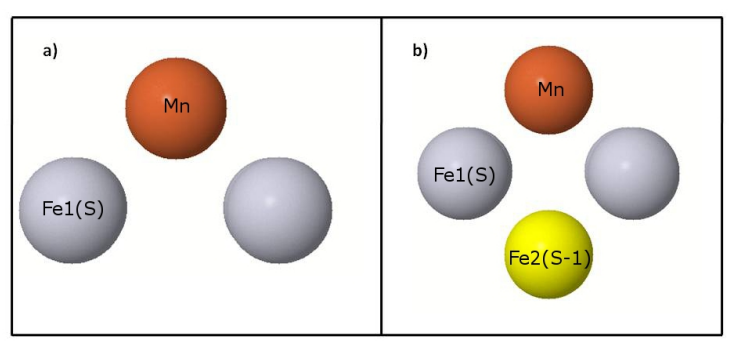

Figura 4.4: Ilustração da primeira (a) e segunda (b) vizinhanças de um adátomo $\mathrm{Mn}$ sobre o $\mathrm{Fe}(001)$. Note-se que no sistema (b) têm-se quatro Fe1(S) e um Fe2(S-1).

Na Tabela 4.4, apresentamos os valores obtidos para o $\mathrm{m}_{s}$ e $\mathrm{m}_{L}$ considerando um adátomo Mn sobre o $\mathrm{Fe}(001)$, em cálculos autoconsistentes com a inclusão de primeiros e segundos vizinhos em torno do sítio de Mn e considerando uma configuração magnética, onde o átomo de Mn tem seu momento magnético paralelo aos momentos dos átomos de Fe do substrato. Pelos dados apresentados na Tabela 4.4, podemos verificar que há uma variação relevante nos valores dos momentos $m_{s}$ com a inclusão da segunda camada de 
vizinhos. Fizemos também testes incluindo os terceiros vizinhos no cálculo autoconsistente. Porém, verificamos que os $m_{s}$ para o adátomo Mn e para os átomos de Fe não são modificados significativamente com a inclusão de terceiros vizinhos. Assim, consideramos que a inclusão de segundos vizinhos é suficiente para descrever corretamente o adátomo Mn sobre o $\mathrm{Fe}(001)$ e, por conseqüência utilizamos este mesmo critério para o estudo de outras nanoestruturas de Mn sobre o Fe(001). Com relação aos $m_{L}$, nossos cálculos indicam que os $m_{L}$ são pequenos comparados aos $m_{s}$ tanto no sítio de $\mathrm{Mn}$, como nos sítios de Fe.

Tabela 4.4: Momento magnético de spin e orbital (em unidades de $\mu_{B}$ ) para o adátomo Mn sobre o Fe(001) em cálculos autoconsistentes obtidos com a inclusão somente de primeiros e segundos vizinhos em torno do sítio de Mn.

\begin{tabular}{|c|c|c|c|}
\hline Configuração & átomo & $\mathrm{m}_{s}$ & $\mathrm{~m}_{L}$ \\
\hline \hline \multirow{3}{*}{ inclusão de $1^{\underline{0}}$ vizinhos } & $\mathrm{Mn}$ & 3,40 & 0,02 \\
& $\mathrm{Fe} 1$ & 2,60 & 0,07 \\
& $\mathrm{Fe} 2$ & 2,60 & 0,07 \\
\hline \multirow{4}{*}{ inclusão de $2^{\underline{0}}$ vizinhos } & $\mathrm{Mn}$ & 3,44 & 0,02 \\
& $\mathrm{Fe} 1$ & 2,62 & 0,07 \\
& $\mathrm{Fe} 2$ & 2,62 & 0,07 \\
& $\mathrm{Fe} 3$ & 2,07 & 0,04 \\
\hline
\end{tabular}

Com o objetivo de investigar o ordenamento magnético deste sistema, isto é, se o momento magnético do Mn apresenta-se paralelo [Fig. 4.5(a)] ou antiparelo [Fig. 4.5(b)] aos momentos magnéticos dos átomos de Fe do substrato, calculamos o acoplamento de troca, $J_{i j}$, entre o adátomo Mn e seus primeiros e segundos vizinhos Fe. Usando a configuração FM como o estado de referência, obtivemos para o acoplamento de troca entre o adátomo Mn e seus quatro primeiros vizinhos Fe $\left[\mathrm{Fe} 1(\mathrm{~S})\right.$ na Fig. 4.4(b)] o valor $J_{M n-F e 1}=$ $-2,7 \mathrm{meV}$, e entre o $\mathrm{Mn}$ e seu segundo vizinho Fe o valor $J_{M n-F e 2}=15$ meV. Levando em conta a multiplicidade de cada sítio podemos calcular a 
constante de troca efetiva para este sistema

$$
J^{e f f}=4 . J_{M n-F e 1}+J_{M n-F e 2}=4,2 m e V .
$$

É interessante notar que a interação é de longo alcance, devido a interação de troca magnética entre segundos vizinhos ser bastante importante e de sinal contrário de primeiros vizinhos, de forma a resultar num estado FM. Comparando a variação de energia total nas duas configurações FM ( $E \uparrow \uparrow)$ e AF $(E \uparrow \downarrow)$, obtemos $\Delta E=E \uparrow \uparrow-E \uparrow \downarrow=-12 \mathrm{meV} /$ átomo. Este valor negativo de $\Delta E$ indica que a magnitude de $E \uparrow \uparrow$ é maior e, portanto, corresponde ao estado de mais baixa energia (negativa), o que corrobora o resultado obtido na análise de $J_{i j}$. Nossos resultados de análise energética para o adátomo Mn sobre o Fe(001) estão em boa concordância com os obtidos por outro método de primeiros princípios baseado na DFT [30].

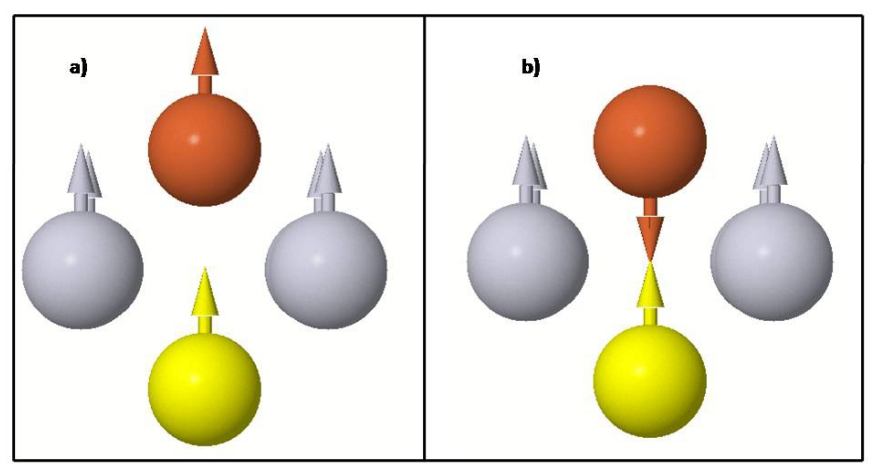

Figura 4.5: Configurações: a) FM e b) AF para o adátomo Mn sobre a superfície Fe(001).

\subsection{2 Átomo de Mn embebido na superfície de $\mathrm{Fe}(001)$}

Nesta seção, apresentamos os resultados para impureza de Mn embebida na superfície $\mathrm{Fe}(001)$ (Fig. 4.6), onde o objetivo principal é procurar compreender como os diferentes aspectos estruturais interferem no comportamento magnético deste sistema, comparado ao adátomo Mn sobre o Fe(001). Substituímos agora um sítio de Fe da camada da superfície, Fe(S), por um 
átomo de Mn e calculamos de forma autoconsistente os parâmetros de potencial para o sítio de Mn e para os seus primeiros e segundos vizinhos Fe e esferas vazias, de forma análoga ao apresentado na seção anterior.

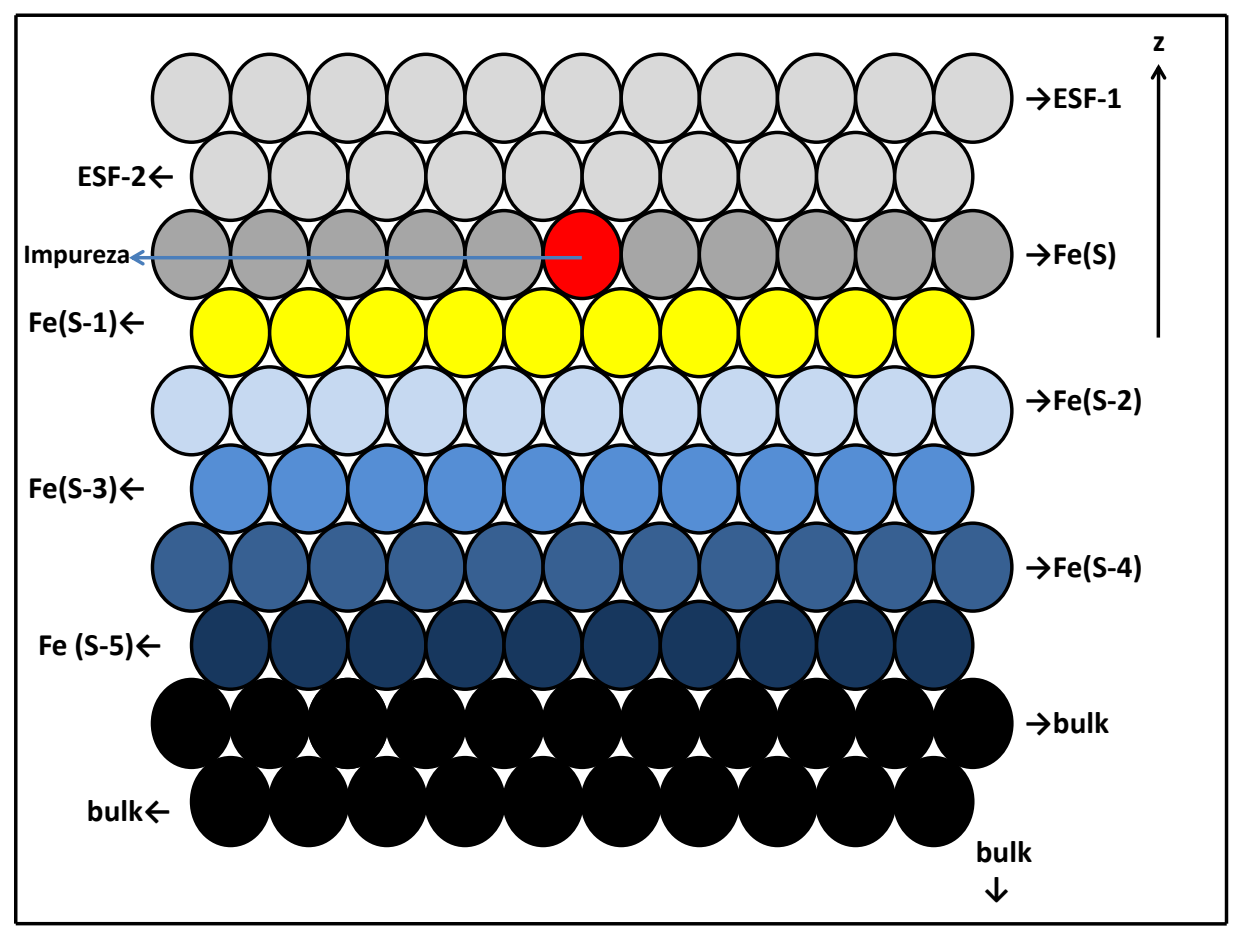

Figura 4.6: Representação esquemática para a impureza de Mn embebida na primeira camada de $\mathrm{Fe}(001)$.

Os valores de $\mathrm{J}_{i j}$ obtidos entre a impureza de Mn na configuração FM e seus quatro primeiros vizinhos $\mathrm{Fe}$ localizados na sub-superfície $\left[\mathrm{Fe}_{1}(\mathrm{~S}-1)\right.$ na Fig. 4.7] são $J_{M n-F e_{1(S-1)}}=-7,4 \mathrm{meV}$, e entre o Mn e seus quatro segundos vizinhos localizados na primeira camada $\left[\mathrm{Fe}_{2}(\mathrm{~S})\right.$ na Fig. 4.7] $J_{M n-F e_{2(S)}}=$ $-10,7 \mathrm{meV}$, e seu segundo vizinho da terceira camada $\left(\mathrm{Fe}_{2}(\mathrm{~S}-2)\right.$ na Fig. 4.7) $J_{M n-F e_{2(S-2)}}=17,9 \mathrm{meV}$. Levando em conta a multiplicidade de cada sítio, podemos calcular a constante de troca efetiva para este sistema

$$
J^{e f f}=4 . J_{M n-F e_{1(S-1)}}+4 . J_{M n-F e_{2(S)}}+J_{M n-F e_{2(S-1)}}=-54,3 \mathrm{meV} .
$$

Nosso resultado para a diferença na energia entre as configurações FM e 
$\mathrm{AF}$ é $\approx 29 \mathrm{meV} /$ átomo em favor da configuração AF, o que corrobora o resultado obtido na análise de $J_{i j}$. Este resultado está em concordância com outro resultado ab initio [30]. Na sua configuração mais estável, o átomo de $\mathrm{Mn}$ embebido tem um $\mathrm{m}_{s_{M n}}=-3,75 \mu_{B}$. Os segundos vizinhos $\mathrm{Fe}$ tem

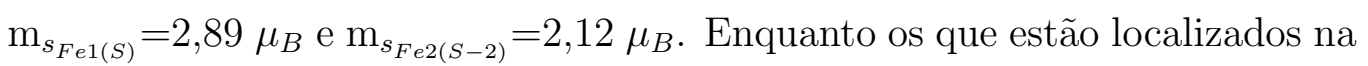
sub-superfície $\mathrm{m}_{s_{F e 1(S-1)}}=1,90 \mu_{B}$.

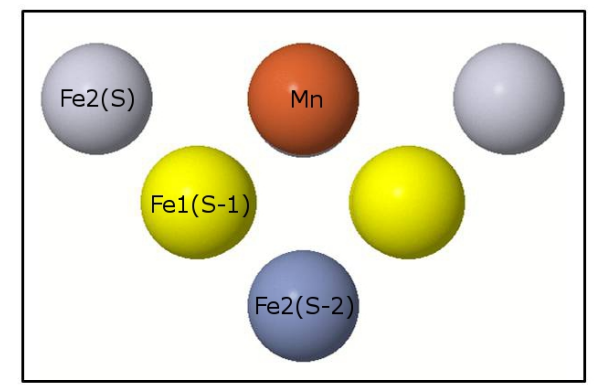

Figura 4.7: Representação esquemática ilustrando a impureza Mn e seus primeiros, [Fe1(S-1)], e segundos vizinhos, [Fe2(S) e Fe2(S-2)]. Note-se que no sistema presente têm-se quatro Fe1(S-1), quatro Fe2(S) e um Fe2(S-2).

Na Fig. 4.8 apresentamos as LDOS para o adátomo FM e impureza AF na superfície Fe(001). Em ambos os casos, o ordenamento magnético é uma conseqüência da interação de troca magnética de longo alcance entre os átomos de Mn e Fe.

\subsubsection{Nanofios de Mn sobre a superfície Fe(001) - Re- sultados colineares}

Apresentamos na Fig.4.9 a geometria das cadeias lineares finitas de Mn $(\mathrm{n} \geq 2)$ adsorvidas ao longo da direção [100] de uma superfície $\mathrm{Fe}(001)$ bcc. Cada átomo de Mn, nestes casos, tem somente átomos de Fe como primeiros vizinhos e os segundos vizinhos são átomos de Mn e Fe, onde a distância entre os átomos de Mn é igual ao parâmetro de rede do Fe $b c c(a=2,87 \AA)$. As configurações dos diferentes nanofios serão denotadas por $\mathrm{Mn}_{n} / \mathrm{Fe}(001)$, onde 

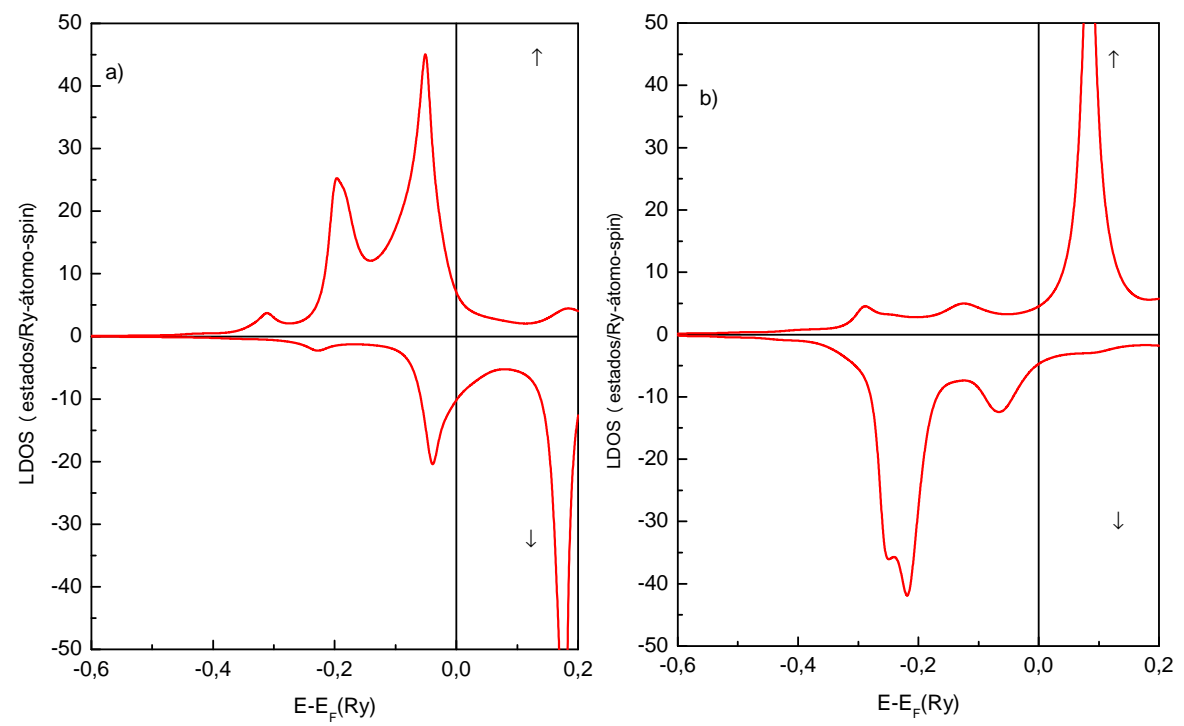

Figura 4.8: Densidades de Estados Locais projetados nos sítios de Mn: (a) adátomo FM e (b) impureza AF em Fe(001)).

$2 \leq n \leq 9$. Realizamos cálculos para diferentes configurações magnéticas colineares dos nanofios de Mn, assumindo que a superfície Fe tem a configuração FM. Foram considerados os seguintes casos (Fig. 4.10): (i)-(FM), onde todos os átomos de Mn e Fe são ferromagneticamente alinhados; (ii)(AF), onde todos os momentos magnéticos do Mn são ferromagneticamente alinhados, mas antiferromagneticamente alinhados em relação aos momentos do Fe; e (iii), onde todos os momentos magnéticos do Mn são antiferromagneticamente alinhados entre si. No caso (iii) foram examinadas três situações: (iii.1)-(FI1), onde o número de átomos Mn é par e a magnetização total do nanofio de Mn é quase nula; (iii.2)-(FI2), onde o número de átomos de Mn é impar e existe um momento magnético total do nanofio de Mn não emparelhado fornecendo uma magnetização resultante alinhada paralelamente a magnetização do Fe; (iii.3)-(FI3) onde o número de átomos de Mn é impar 
e existe um momento magnético do Mn não emparelhado fornecendo uma magnetização resultante alinhada antiparalela a magnetização do Fe.

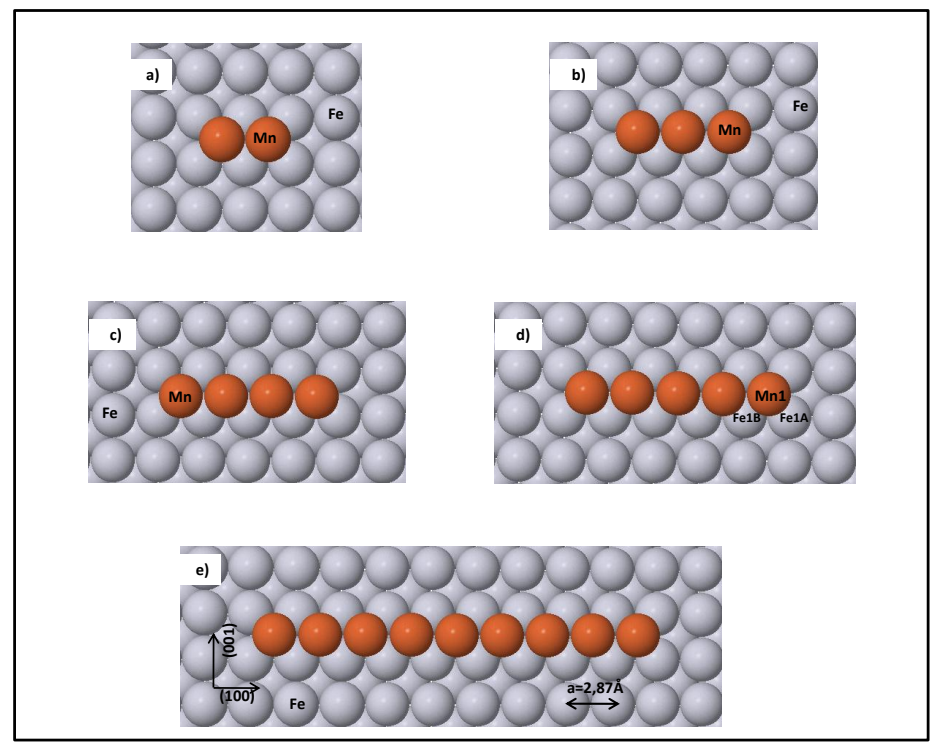

Figura 4.9: Ilustração das cadeias lineares finitas aqui estudadas contendo (a) 2, (b) 3, (c) 4, (d) 5 e (e) 9 átomos de Mn adsorvidos na superfície Fe(001): átomos de $\mathrm{Mn}$ em vermelho e átomos de Fe em cinza.

Nossos cálculos indicam que as contribuições de spin para os momentos magnéticos do Mn variam consideravelmente. Para o caso (i)-(FM), obtivemos $\mathrm{m}_{s_{M n}}=3,4 \mu_{B}$ para o adátomo Mn (Seção 4.4.1), $\mathrm{m}_{s_{M n}} \approx 3,3 \mu_{B}$ para átomos de $\mathrm{Mn}$ localizados nas extremidades da cadeia e $\mathrm{m}_{s_{M n}} \approx 3,2 \mu_{B}$ para sítios de Mn localizados na parte interna da cadeia. Nossos resultados quando os momentos magnéticos dos sítios de Mn são acoplados (ii)-(AF) são sistematicamente maiores que os obtidos no caso (i)-(FM) e variam de $\mathrm{m}_{s_{M n}} \approx 3,5 \mu_{B}$, para átomos de Mn localizados nas extremidades da cadeia, até $\mathrm{m}_{s_{M n}} \approx 3,6 \mu_{B}$, para átomos de Mn localizados na parte interna da cadeia linear. Os momentos magnéticos de spin correspondentes aos casos estudados para os átomos de Fe localizados na superfície (S) com um primeiro e dois primeiros vizinhos $\mathrm{Mn}$ (Figs. 4.9 e 4.11) são $\mathrm{m}_{s_{F e}} \approx 2,6 \mu_{B}$ e $\mathrm{m}_{s_{F e}} \approx$ $2,2 \mu_{B}$, respectivamente. Para átomos de Fe localizados na segunda camada 


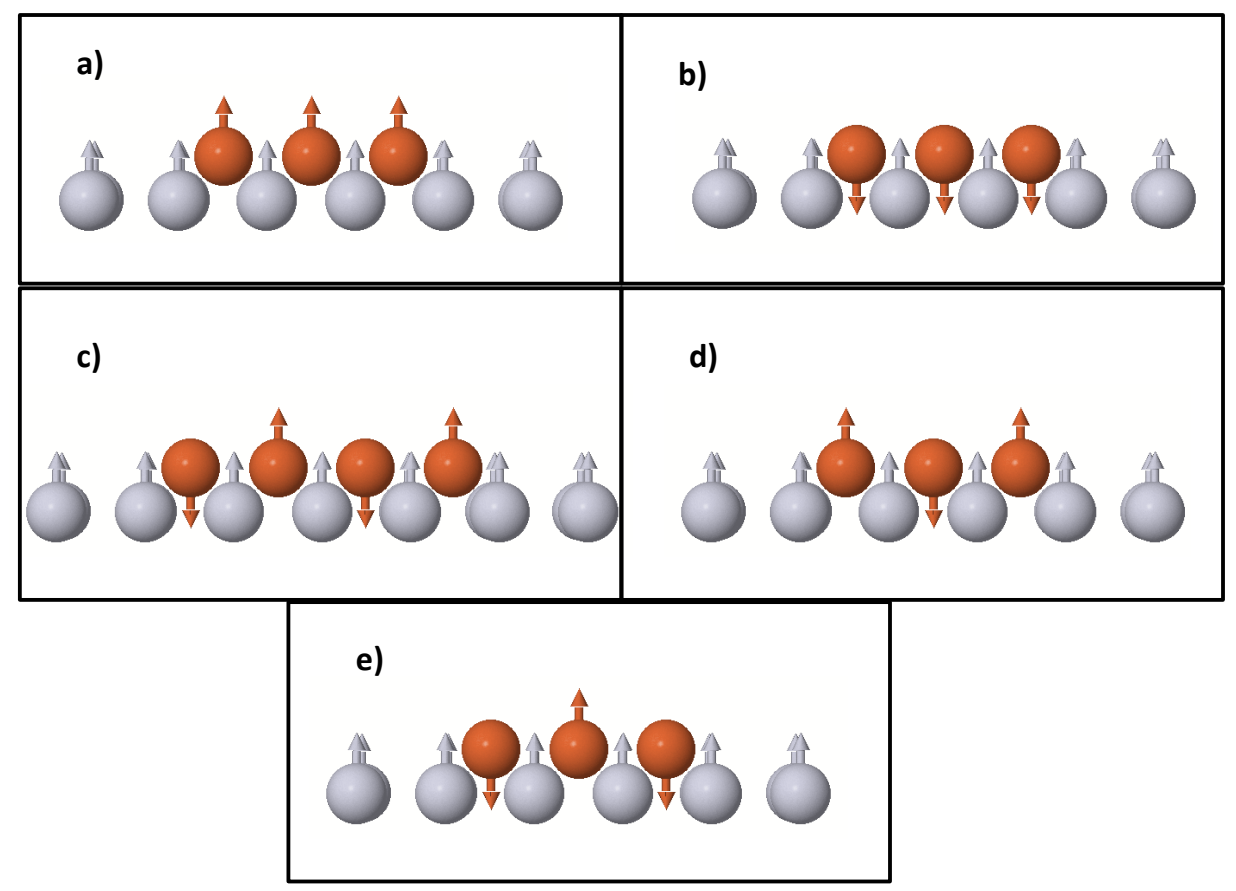

Figura 4.10: Ilustração das diferentes configurações magnéticas colineares usadas neste trabalho para os nanofios de Mn em Fe(001): (a) FM, (b) AF, (c) FI1, (d) FI2 e (e) FI3.

da superfície $\mathrm{Fe}(\mathrm{S}-1)$ são $\mathrm{m}_{s_{F e}} \approx 2,1 \mu_{B}$.

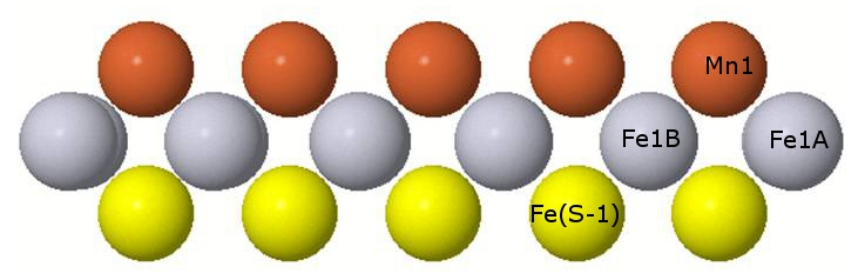

Figura 4.11: Ilustração de um nanofio $\mathrm{Mn}_{5} / \mathrm{Fe}(001)$, onde são ilustrados os átomos de Fe com um primeiro (Fe1A) e dois primeiros vizinhos (Fe1B) Mn. Também são ilustrados os átomos de Fe segundos vizinhos (esferas amarelas) do Mn. 
A análise energética de estabilidade magnética no caso do dímero de Mn adsorvido em $\mathrm{Fe}(001)$ (Fig. 4.9(a)) indica que a diferença entre as configurações (i)-(FM), (ii)-(AF), e (iii.1)-(FI1) é pequena: $\approx 3 \mathrm{meV} /$ átomo. Para o trímero de Mn assim como para maiores cadeias de Mn foram também obtidas várias configurações magnéticas colineares. Estes estados estáveis e metaestáveis correspondem mínimos de energias locais, onde as diferenças de energia total são de poucos meV. Dentro deste pequeno intervalo de energia, as cadeias com um número par de átomos apresentam a configuração magnética (iii.1)-(FI1) com mais baixa energia e naquelas com número ímpar de átomos de Mn a configuração magnética de mais baixa energia é (iii.2)(FI2).

Realizamos também cálculos para o dímero e o trímero de Mn colocados na direção [110] sobre a superfície Fe(001) (Fig. 4.12). Neste caso, a distância interatômica é $\mathrm{d}=\sqrt{2} \mathrm{a}$; obtemos pela análise energética que ambas as configurações (i)-(FM) e (iii.1)-(FI1) são magneticamente estáveis para o dímero. Enquanto a configuração (iii.2)-(FI2) é o estado mais estável para o trímero, mas com energias totais muito próximas a outros estados colineares.
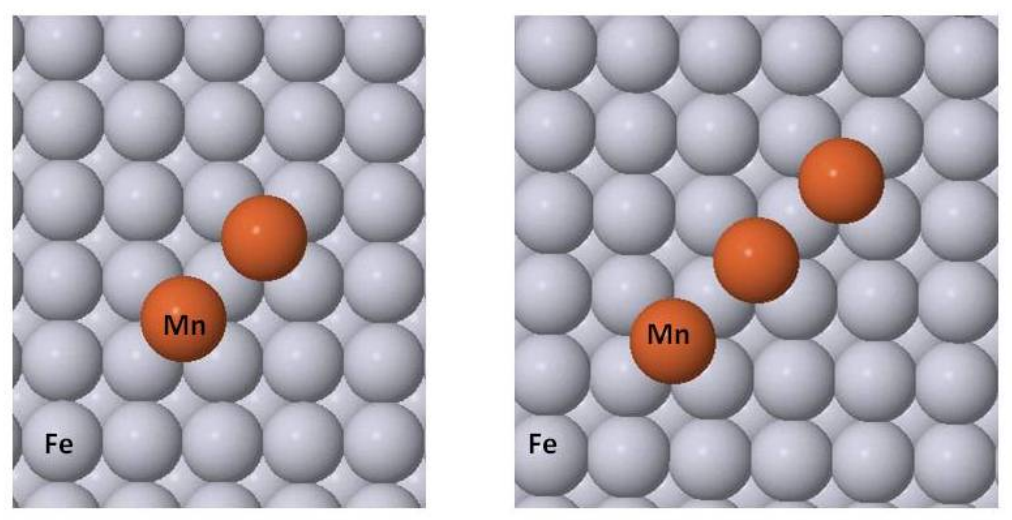

Figura 4.12: Dímero e trímero de Mn depositados em forma diagonal na superfície $\mathrm{Fe}(001)$.

Para a análise destes resultados, mostramos na Fig. 4.13 os valores das 
interações de troca magnética entre os momentos magnéticos dos átomos de $\mathrm{Mn}\left(\mathrm{J}_{M n-M n}\right)$ e entre os momentos magnéticos dos átomos de $\mathrm{Mn}$ e Fe $\left(\mathrm{J}_{M n-F e}\right)$ para as várias cadeias lineares de $\mathrm{Mn}$. Todos os valores foram obtidos a partir da configuração FM. Também fizemos cálculos da interação de troca magnética entre os momentos magnéticos dos Fe primeiros vizinhos e verificamos que $J_{F e-F e}$ é sempre forte e positivo, $\approx 30 \mathrm{meV}$, decrescendo rapidamente para vizinhos mais distantes. Entretanto, podemos ver na Fig. 4.13 que tanto $\mathrm{J}_{M n-M n}$ quanto $\mathrm{J}_{M n-F e}$ são ainda fortes para separação interatômica além de primeiros vizinhos. É interessante notar, que nestes sistemas que $\mathrm{J}_{M n-F e}$ depende não somente da distância interatômica, mas também do número de átomos de Mn que são primeiros vizinhos do átomo de Fe. Por exemplo, para átomos de Fe com somente um primeiro vizinho $\mathrm{Mn}$, o $\mathrm{J}_{M n-F e}$ é negativo e nos casos onde o Fe tem dois primeiros vizinhos $\mathrm{Mn}, \mathrm{J}_{M n-F e}$ é positivo. Usando como exemplo cinco átomos de Mn (Fig. 4.11), a interação de troca entre o momento magnético do Mn localizado na extremidade da cadeia de Mn e seu primeiro vizinho Fe situado na primeira camada da superfície (que tem um único vizinho $\mathrm{Mn}$ ) $\mathrm{J}_{M n 1-F e 1 A}=-1,4 \mathrm{meV}$; por outro lado, o acoplamento de troca deste mesmo átomo de Mn e outro primeiro vizinho $\mathrm{Fe}$ (que tem dois primeiros vizinhos $\mathrm{Mn}$ ) é $\mathrm{J}_{M n 1-F e 1 B}=10,9$ meV. Também, notamos na Fig. 4.13 (a) que a interação de troca entre o momento magnético do átomo de Mn e seu segundo vizinho é sempre FM, e de mesma ordem de magnitude (em algumas outras situações, maior) que o acoplamento de troca entre os primeiros vizinhos Mn-Fe, embora com diferentes sinais em alguns casos. Deste modo, um simples modelo de Heisenberg que considera apenas as interações de primeiros vizinhos é claramente inapropriado para descrever estes sistemas. A análise das propriedades magnéticas requer assim um modelo mais apropriado, que considere uma variedade de interações entre pares mais distantes e suas dependências com os tipos de vizinhanças. 

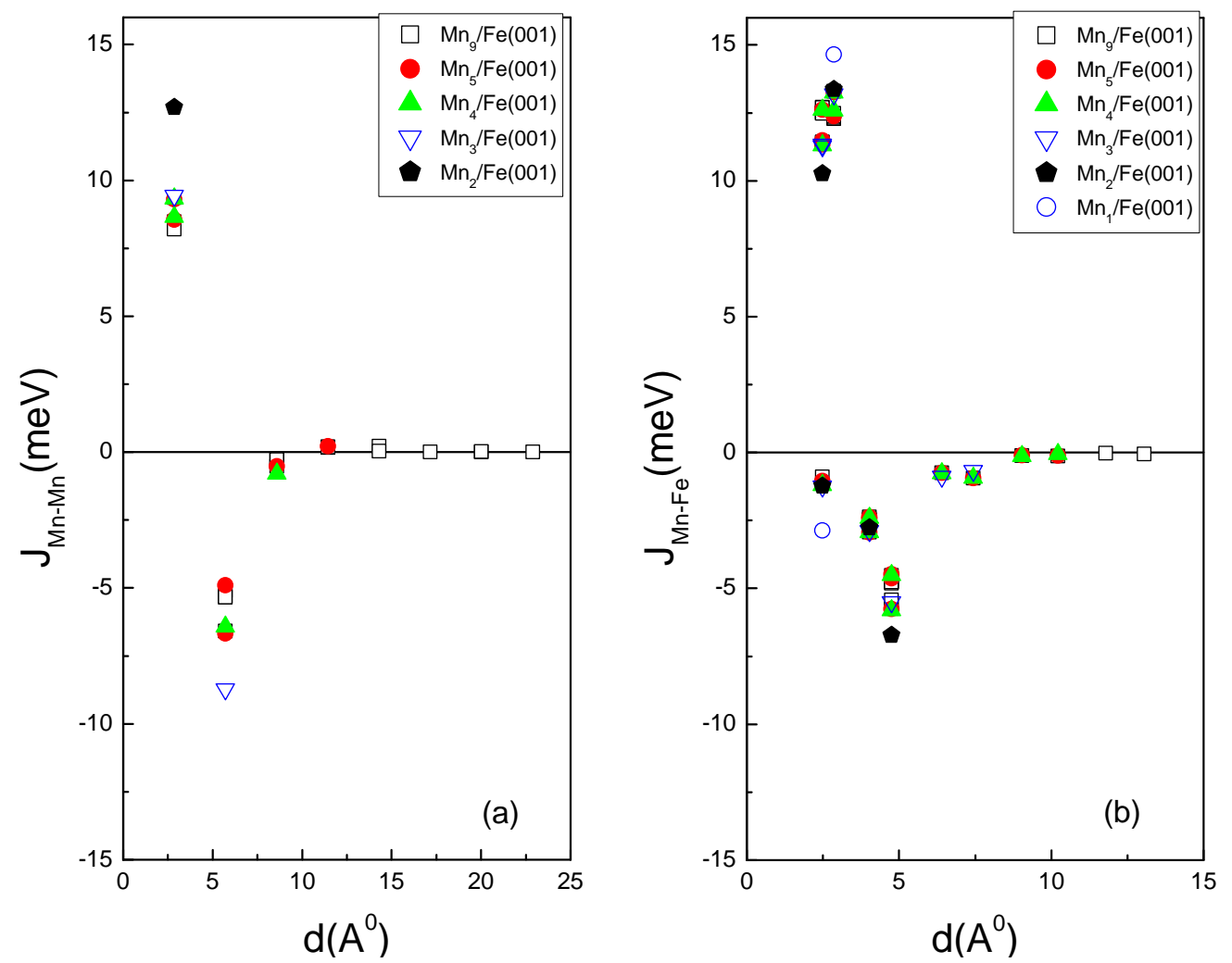

Figura 4.13: Acoplamentos de troca $J_{i j}$ em função da distância envolvendo os nanofios de $\mathrm{Mn}_{n} / \mathrm{Fe}(001)$ : (a) entre os momentos magnéticos dos átomos de $\mathrm{Mn}, \mathrm{J}_{M n-M n}$, e (b) átomos de $\mathrm{Mn}$ e Fe, $\mathrm{J}_{M n-F e}$.

\subsubsection{Efeitos de relaxação}

Investigamos os efeitos de relaxação estrutural nas propriedades magnéticas de alguns nanofios de Mn adsorvidos em Fe(001), a saber, um único átomo de Mn, dímero de Mn e trímero de Mn. Portanto, recalculamos a estrutura eletrônica, momentos magnéticos e configurações magnéticas mais estáveis considerando variações de $\pm 6 \%$ e de $\pm 2 \%$ na distância entre a nanoestrutura de Mn e a superfície Fe(001). Nossos resultados indicam que o momento magnético calculado não sofre grandes variações com a relaxação estrutural. O momento magnético local do Mn muda menos que $0,1 \mu_{B}$ e os momen- 
tos magnéticos dos átomos de Fe localizados na camada superficial variam no intervalo entre 0,05 a $0,15 \mu_{B}$, com valores que decrescem sobre compressão e aumentam com a expansão. Os momentos magnéticos dos átomos de Fe com uma quantidade maior de primeiros vizinhos Mn apresentam uma maior variação e os átomos de Fe localizados na $\mathrm{Fe}(\mathrm{S}-1)$ não mudam significativamente. As interações de troca $\mathrm{J}_{M n-M n}$ e $\mathrm{J}_{M n-F e}$, por outro lado, são fortemente afetadas pelos efeitos de relaxação, mas não há variação em seus sinais. Desta maneira, as configurações magnéticas tanto do dímero quanto do trímero são mantidas, quando as relaxações de $\pm 6 \%$ são consideradas. A única exceção é o caso do adátomo Mn em Fe(001), onde afastando-se da superfície $(6 \%)$ as configurações FM e AF se tornam degeneradas.

\subsubsection{Efeitos de Interdifusão}

Também analisamos o efeito de interdifusão do Mn utilizando os casos do dímero e trímero de Mn. Para tanto, consideramos que um átomo de Mn foi colocado como impureza na primeira camada de Fe e os outros átomos de Mn foram colocados como adátomos na superfície Fe(001). Os átomos de Mn são posicionados, nestes casos, como primeiros vizinhos. A interação de troca entre os momentos magnéticos do adátomo e impureza é forte e AF. Sendo $\mathrm{J}_{M n-M n}=-102 \mathrm{meV}$ e $\mathrm{J}_{M n-M n}=-54 \mathrm{meV}$ para o dímero e trímero de $\mathrm{Mn}$, respectivamente. Estas interações são mais fortes que quaisquer outras interações Mn-Mn e Mn-Fe e levam a configurações magnéticas mais estáveis mostradas na Fig. 4.14. A diferença em energia entre estes estados mais estáveis e outras possíveis configurações colineares são maiores que $20 \mathrm{meV} /$ átomo. Nossos resultados estão de acordos com medidas de $X$-ray magnetic circular dichroism (XMCD), onde foi verificado que os momentos dos átomos de Mn embebidos na primeira camada de Fe(001) são acoplados antiparalelamente à direção dos momentos magnéticos do Fe [17].

A existência de vários ordenamentos magnéticos colineares estáveis para os nanofios de Mn adsorvidos em Fe(001), sem considerar a interdifusão, indicam uma possível relação com a presença de estruturas de spin estáveis com 


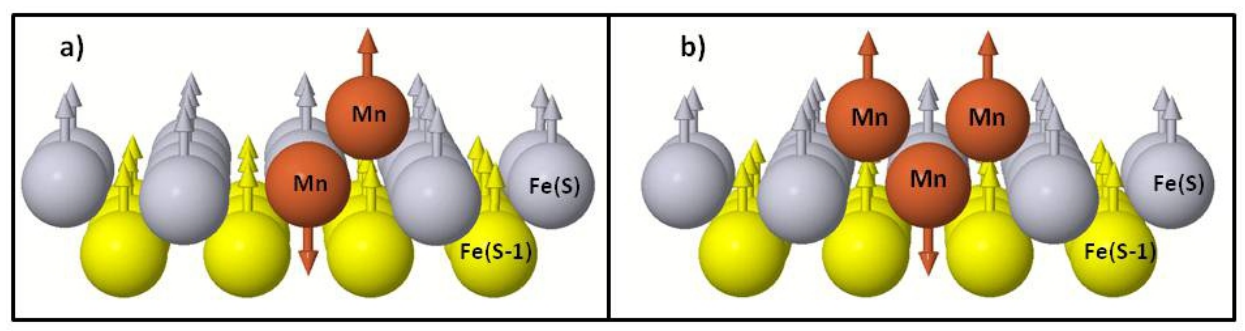

Figura 4.14: Configurações magnéticas obtidas para: (a) dímero e (b) trímero, onde um átomo de Mn é embebido na primeira camada de Fe e os outros (um ou dois átomos de Mn) são colocados como adátomos.

topologia não trivial [113-116]. Notamos também que similarmente, resultados teóricos anteriores revelam configurações magnéticas não colineares para um, dois [22] e também seis [45] camadas de Mn depositadas em Fe(001).

\subsubsection{Resultados não colineares}

As interações de troca de longo alcance e as competições entre as interações de pares FM e AF sugerem que as configurações magnéticas dos nanofios de Mn adsorvidas em Fe(001) podem ser não colineares. Foi explorada esta possibilidade fazendo cálculos não colineares nos nanofios mostrados nas Figs.4.9(c)-(e), com a inclusão do acoplamento spin-órbita. Nossos resultados são mostrados nas Figs.4.15(a)-(d), onde os momentos magnéticos do substrato de Fe (não mostrados) são orientados para fora do plano. Estas configurações podem ser vistas como tendo os momentos magnéticos do Mn aproximadamente um ordenamento magnético FM não colinear entre si e AF entre os Fe primeiros vizinhos do Mn. Estes resultados estão de acordo com resultados experimentais para sub-monocamadas de Mn em uma superfície Fe(001) [17-19]. Também é interessante notar que as energias dos estados não colineares, mostradas nas Figs.4.15(a)-(d), são semelhantes aos correspondentes estados colineares, diferindo por poucos meV/átomo. Os resultados apresentados nas Figs. 4.15(c) e (d) indicam que o ordenamento magnético 
para estes sistemas pode ser bastante interessante, particularmente para longos nanofios. Nossos cálculos indicam diferentes orientações magnéticas para os átomos de Fe na superfície que tem primeiros vizinhos Mn quando consideramos cálculos com e sem a inclusão de spin-órbita. Sem inclusão de spinórbita, mostrado na Fig. 4.15(e), foi obtida uma onda de spin, caracterizada por um modulação senoidal com um período de $\approx 2,5 \mathrm{~nm}$, que corresponde ao comprimento do nanofio de Mn. Os momentos magnéticos dos átomos de Fe na sub-superfície (S-1) apresentam um comportamento similar, mas com menor magnitude. As configurações magnéticas não colineares dos momentos magnéticos do Mn assemelham-se a uma onda amortecida, com os momentos magnéticos do Mn que estão nos extremos acoplados AF com os primeiros vizinhos Fe.

Cálculos para longos nanofios, que levam em consideração o acoplamento spin-órbita, mostram uma rotação de spin, mostrado na Fig. 4.15(f), que pode ser causada por contribuições não desprezíveis das interações de Dzyaloshinskii-Moriya [82,83]. O nanofio de Mn e seus primeiros vizinhos Fe têm um ordenamento magnético de spin espiral helicoidal [117], com um período aproximadamente igual ao comprimento do fio de Mn.

Para sistemas com interdifusão de Mn (Fig. 4.14), nossos cálculos indicam que existe um alinhamento antiferromagnético não colinear entre o Mn embebido e o adsorvido. As direções dos momentos magnéticos de Fe primeiros vizinhos são quase perpendiculares aos momentos magnéticos do Mn (Fig. 4.16). Contudo, nossos cálculos indicam que as configurações magnéticas não colineares tem energias maiores em comparação com as configurações magnéticas colineares $(\approx 20 \mathrm{meV}$ para o dímero e $\approx 100 \mathrm{meV}$ para o trímero), indicando que as interdifusões de Mn podem estabilizar em configurações colineares. É interessante notar que não foram obtidos para estes sistemas os efeitos de paridade calculados por S. Lounis et al., Ref. [118] para nanofios de Mn adsorvidos em uma superfície fcc de Fe(001), onde as cadeias com um número par de átomos de Mn sempre exibem um magnetismo não colinear, em contraste com cadeias com números impares de átomos de $\mathrm{Mn}$ que apresentam um configuração colinear ferrimagnética. Assim, esta ca- 

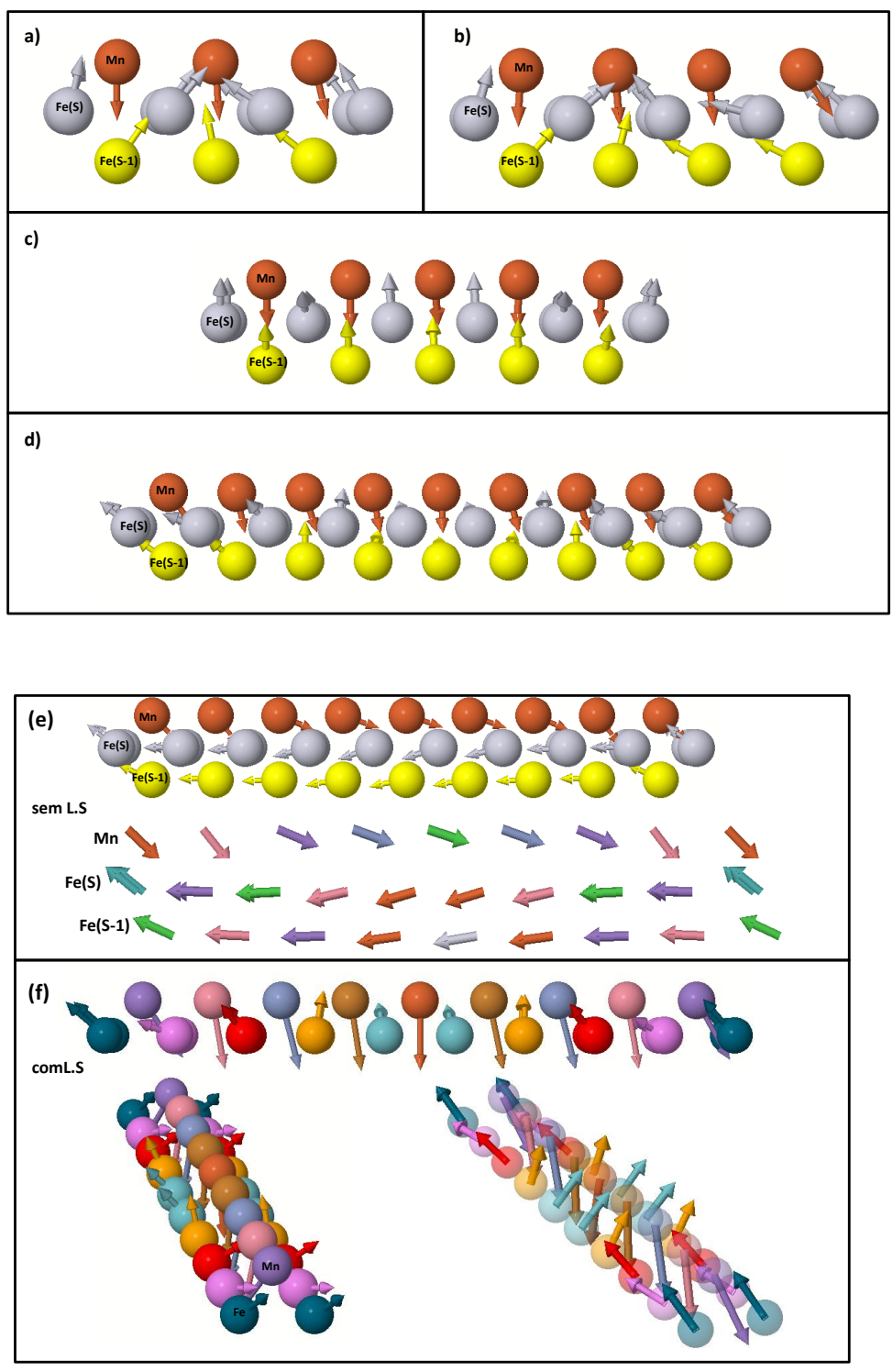

Figura 4.15: Configurações magnéticas não colineares para os nanofios: (a) $\mathrm{Mn}_{3}$, (b) $\mathrm{Mn}_{4}$, (c) $\mathrm{Mn}_{5}$, e (d) $\mathrm{Mn}_{9}$ em Fe(001). Configuração magnética para o nanofio $\mathrm{Mn}_{9}$ sem (e) e com (f) acoplamento spin-órbita. Em (f) as setas são proporcionais ao momento magnético local.

racterística magnética distinta apresentada pelos nanofios de Mn adsorvidos em uma superfície Fe(001) fcc e bcc surgem de diferentes competições de 
interações de troca magnética que ocorrem nestes dois sistemas.

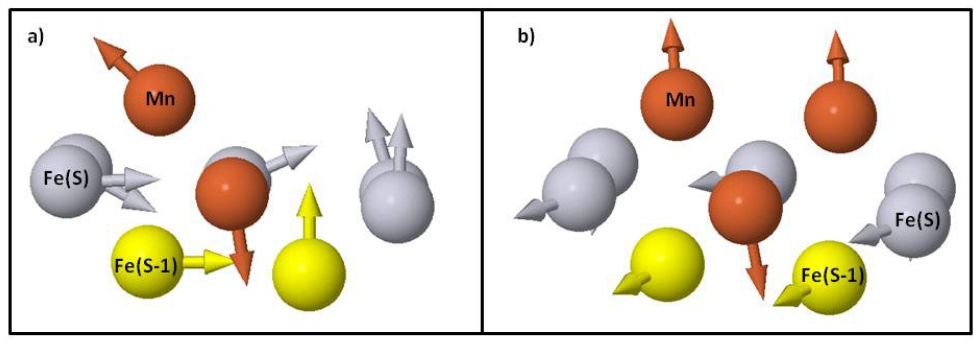

Figura 4.16: Configurações magnéticas não colineares obtidas para os sistemas com Mn embebido em Fe(001): a) dímero e b) trímero de Mn.

\subsection{Nanoestruturas bi-tridimensionais de Mn sobre a superfície de $\mathrm{Fe}(001)$}

A morfologia de nanoestruturas de Mn adsorvidas em Fe(001) podem ter um papel importante em suas características magnéticas. Para a investigação destes efeitos, realizamos cálculos incluindo o acoplamento spin-órbita para ilhas (arranjos bidimensionais) contendo (Fig. 4.17) quatro (quadrado), cinco (cruz) e seis (retângulo) átomos de Mn, além de aglomerados tridimensionais em forma de pirâmide (Fig. 4.18) contendo cinco átomos de Mn. Notamos inicialmente que as competições entre as interações de troca magnética e suas características de longo alcance fornecem vários mínimos locais para a energia e que as energias dos estados magnéticos não colineares, mostrados na Fig.4.17, são muito semelhantes às associadas aos estados colineares correspondentes [Figs. 4.17-(a2)-(b2)-(c2) e 4.17-(a3)-(b3)-(c3)]. Para a ilha de Mn em forma de quadrado obtivemos que o estado magnético mais estável é a configuração colinear ferrimagnética c(2x2) [mostrada na Fig. 4.17-(a2)], contudo, aproximadamente degenerado com a configuração $\mathrm{p}(2 \times 2) \uparrow$ (onde o rótulo $\uparrow$ indica que a maioria dos momentos magnéticos do Mn é paralela à magnetização do substrato de Fe). Um comportamento semelhante foi 
verificado para o aglomerado de Mn com forma de retângulo retratado na Fig. 4.17(b2). Para a ilha de Mn com cinco átomos de Mn [Fig. 4.17(c2)], a configuração colinear mais estável tem somente o momento magnético do Mn central alinhado antiparalelamente aos outros momentos magnéticos do Mn.

Nestes cálculos não colineares paras as ilhas quadradas e retangulares de Mn , obtivemos configurações magnéticas estáveis onde as orientações do Fe e Mn primeiros vizinhos são quase antiparalelas, quando os átomos de Fe tem um único vizinho Mn [Figs. 4.17(a3) e 4.17(b3)] e quase perpendiculares quando o Fe tem dois primeiros vizinhos $\mathrm{Mn}$. O arranjo em forma de cruz mostrado na Fig. 4.17(c3) apresenta um ordenamento magnético não colinear. Um resultado bastante interessante do presente trabalho é o fato de que a estrutura magnética não trivial, dado que os momentos magnéticos do Mn e Fe têm uma rotação para fora do plano, em uma configuração de spin do tipo "flor de lótus", que se assemelha a um half-skyrmion, isto é, um meron, um objeto topológico $[115,119]$. Também é interessante notar que Heinze et al. [120] mostraram a existência de uma rede quadrada bidimensional de skyrmions como estado fundamental magnético em filmes de Fe depositados na superfície $\operatorname{Ir}(111)$, sendo que a origem microscópica foi explicada em termos das interações de troca de Dzyaloshinskii-Moriya. Nosso resultado de uma estrutura magnética complexa como a apresentada na Fig. 4.17(c3) requer assim uma análise que não pode ser realizada no presente trabalho e seria objeto de futuras investigações.

As configurações magnéticas complexas obtidas podem ser explicadas pelas interações de troca magnética que envolve pares dos átomos de Mn $\left(J_{M n-M n}\right)$ bem como a interação de troca entre um átomo de Mn e seus vizinhos Fe $\left(J_{M n-F e}\right)$. Na Fig. 4.19, apresentamos em (a) e (c) a interação de troca magnética $J_{M n-M n}$ e $J_{M n-F e}$ como função da distância interatômica $d$, onde estes resultados foram obtidos através da configuração FM. De forma semelhante aos nanofios de Mn, foram obtidos os seguintes resultados: (i) O acoplamento de troca entre os Fe primeiros vizinhos $\left(J_{F e-F e}\right)$ é forte e positivo, e decresce rapidamente para vizinhos mais distantes; (ii) $J_{M n-M n}$ 


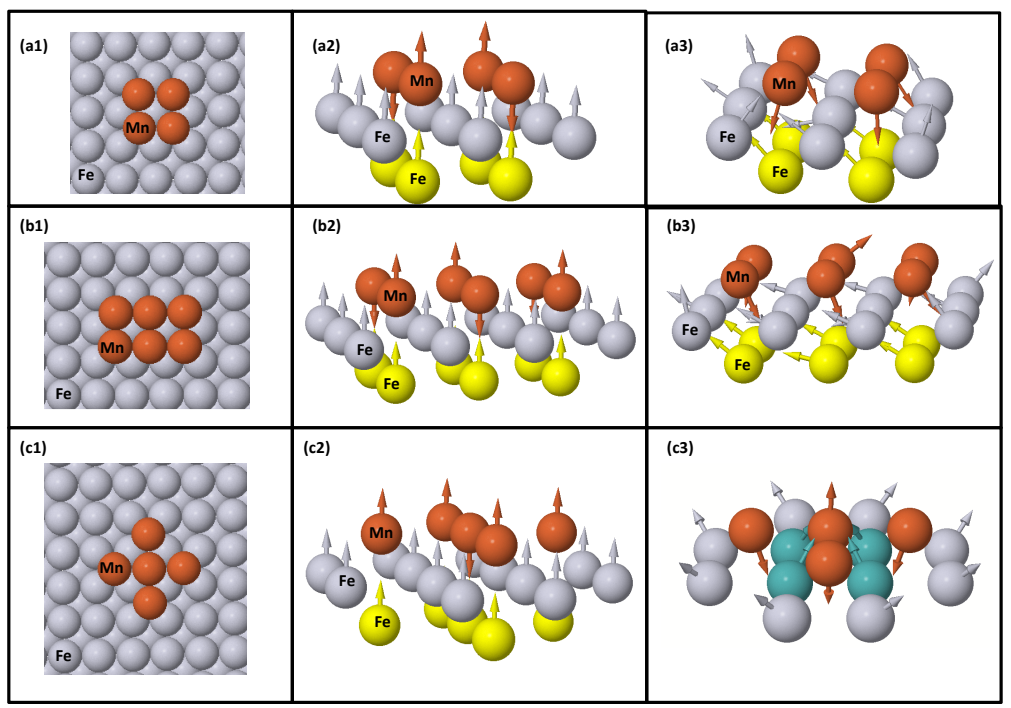

Figura 4.17: Configurações magnéticas para ilhas de Mn com (a) quatro (quadrado), (b) seis (retângulo), e (c) cinco (cruz) átomos em Fe(001). Os estados colineares são mostrados em (a1), (b1), e (c1) e seus correspondentes estados não colineares em (a3), (b3) e (c3). As esferas em azul em (c3) indicam os átomos de Fe próximos ao Mn central.

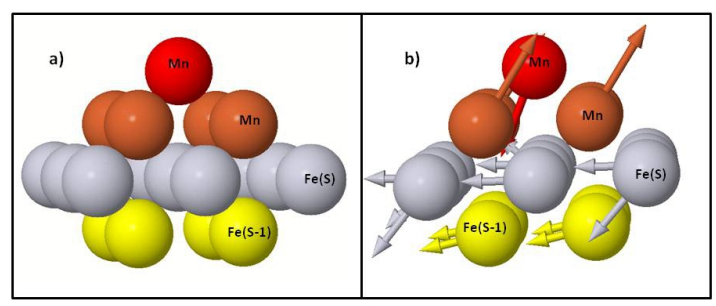

Figura 4.18: Configurações magnéticas para o aglomerado de forma piramidal adsorvido em $\mathrm{Fe}(001)$. As setas são proporcionais aos momentos magnéticos locais. 
e $J_{M n-F e}$ tem um longo alcance e para alguns casos suas magnitudes para momento magnéticos primeiros vizinhos são menores que para vizinhos mais distantes; (iii) $J_{M n-F e}$ não depende somente da distância interatômica MnFe, mas também do número de vizinhos Mn do átomo de Fe. Na Fig. 4.19(d) $J_{M n-F e}$ é apresentado em função do número de primeiros vizinhos Mn que o Fe tem. Verificamos que o valor de $\mathrm{J}_{M n-F e}$ entre primeiros vizinhos é negativo se o átomo de Fe tem somente um único átomo de Mn como primeiro vizinho, e positivo quando o Fe tem mais que um primeiro vizinho $\mathrm{Mn}$.

Também verificamos que $\mathrm{J}_{M n-M n}$ é muito sensível ao número de primeiro mais segundos vizinhos $\mathrm{Mn}\left(N_{M n}\right)$ (no plano). Este fato pode ser visto na Fig. 4.19(a), onde podemos observar que os valores de $\mathrm{J}_{M n-M n}$ têm um intervalo que varia de $-2,7 \mathrm{meV}$ a $+8,2 \mathrm{meV}$ para átomos de $\mathrm{Mn}$ que estão separados por uma distância de um parâmetro de rede. Na Fig. 4.19(c) vemos que $\mathrm{J}_{M n-M n}$ entre os átomos de $\mathrm{Mn}$ separados por uma mesma distância interatômica $a$, decrescem rapidamente com $\mathrm{N}_{M n}$. Uma sensível dependência da interação de troca nas nanoestruras de Mn com o seu meio também foi verificada para nanoestruturas livres $[121,122]$ e depositadas em substratos não magnéticos [121,123-125]. Os valores obtidos para $\mathrm{J}_{M n-F e}$ e $\mathrm{J}_{M n-M n}$ possibilitam uma explicação para transição da configuração magnética observada para sub-monocamadas de Mn adsorvidas em Fe(001). Como podemos ver na Figs. 4.19(b)-(d), para baixas coberturas de Mn (pequeno número de vizinhos Mn), o acoplamento FM Mn-Mn e AF Mn-Fe prevalece. Este fato é consistente com o observado para este limite, isto é, que os momentos magnéticos dos átomos de Mn são FM acoplados, mas AF acoplados com a magnetização do Fe [17-19]. Para altas coberturas de Mn, ou seja, quando o número de vizinhos de Mn aumenta, o acoplamento Mn-Mn muda para AF (Fig. 4.19(b)), e o acoplamento Mn-Fe primeiros vizinhos muda para FM (Fig. 4.19(d)). Assim, existem competições entre estas duas interações magnéticas. Este fato pode levar a uma configuração AF não colinear, como observado para uma monocamada de Mn em Fe(001), onde os momentos magnéticos do Mn são quase perpendiculares à magnetização do substrato [16]. 

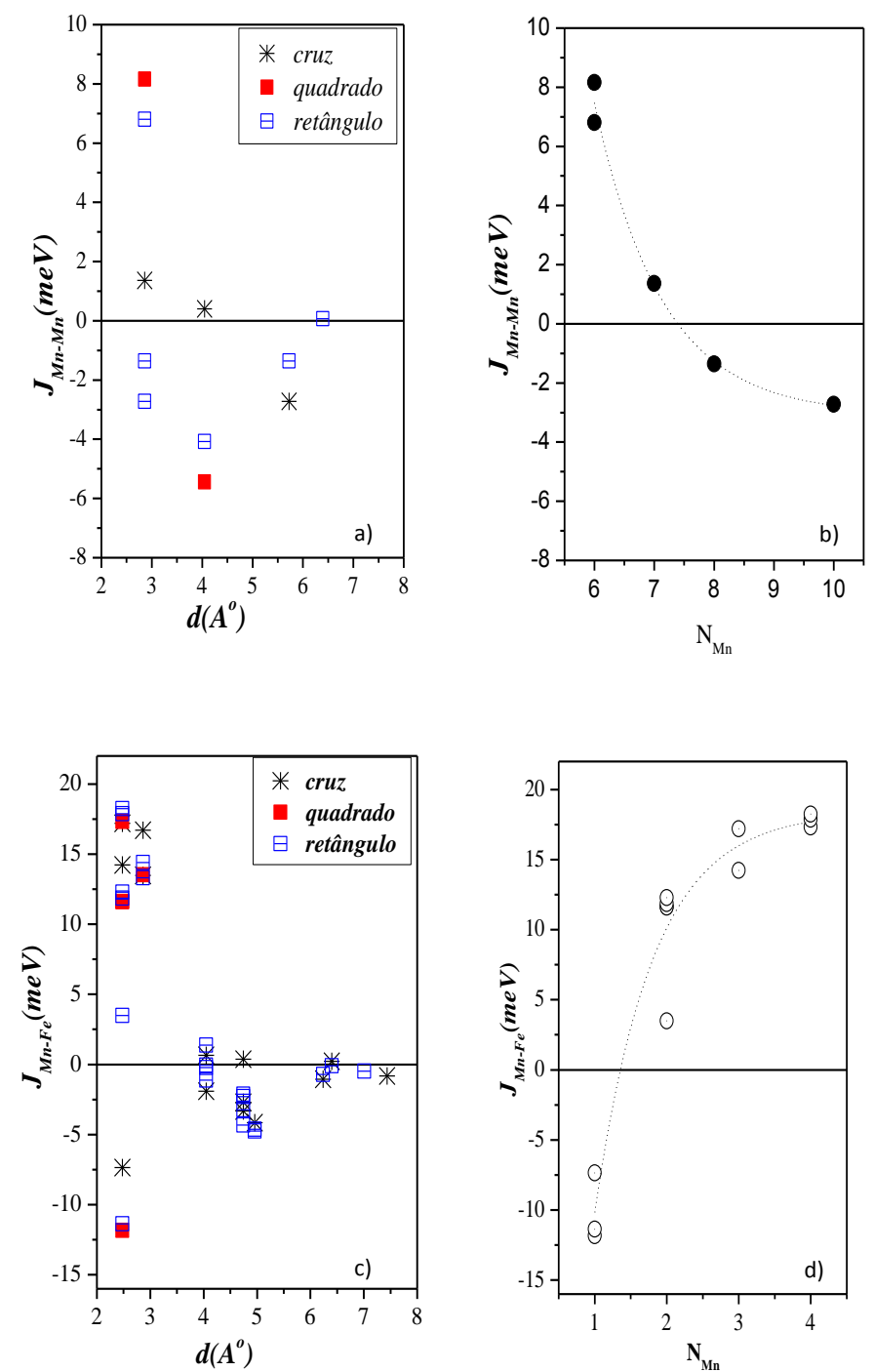

Figura 4.19: Interação de troca $J_{i j}$ entre pares calculados como função da distância interatômica $d$ para diferentes ilhas de Mn adsorvidas em Fe(001) apresentadas na Fig. 4.17: (a) $\mathrm{J}_{M n-M n}$ e (c) $\mathrm{J}_{M n-F e}$. O gráficos (b) mostra os valores de $J_{M n-M n}$ entre sítios primeiros vizinhos em função de $N_{M n}$ (número de primeiros mais segundos vizinhos $\mathrm{Mn}$ ). O gráfico (d) mostra os valores de $J_{M n-F e}$ entre sítios primeiros vizinhos em função do número de primeiros vizinhos $\mathrm{Mn}$. 
Para o aglomerado de Mn com forma de pirâmide adsorvida em Fe(001), mostrado na Fig. 4.18(a), o estado magnético colinear mais estável exibe um alinhamento FM dos momentos magnéticos do Mn localizados na mesma camada, bem como entre os momentos magnéticos do Mn e Fe na interface, mas ocorre um ordenamento antiparalelo entre os momentos magnéticos dos Mn situados em diferentes camadas. A configuração magnética do estado fundamental obtido nos cálculos não colineares é mostrada na Fig. 4.18. O momento magnético do átomo de Mn situado no topo da pirâmide é quase antiparalelo aos momentos magnéticos dos Mn localizados na interface com o Fe da superfície. Estes momentos magnéticos do Mn são quase perpendiculares aos momentos magnéticos dos vizinhos Fe, em concordância com os resultados experimentais [95,96] e teóricos [45] para monocamadas de Mn em $\mathrm{Fe}(001)$. A interação de troca calculada entre primeiros vizinhos Mn [Fig. 4.20-(a)] é negativa e muito grande em módulo, favorecendo assim um forte acoplamento AF entre eles. A amplitude de $\mathrm{J}_{M n-M n}$ decresce, oscilando entre FM e AF, quando a distância interatômica entre sítios de Mn localizados na mesma camada aumenta. As interações de troca entre os átomos de Mn e Fe (Fig. 4.20)-(b) na interface são ferromagnéticas para primeiros vizinhos, mas tornam-se menores e antiferromagnéticas para vizinhos mais distantes.
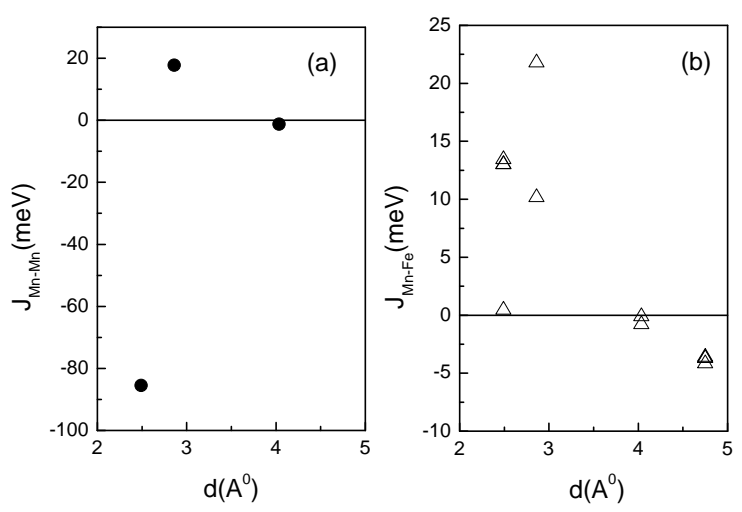

Figura 4.20: Interação de troca magnética como função da distância entre os sítios para os átomos mostrados na Fig. 4.18: (a) $J_{M n-M n}$ e (b) $J_{M n-F e}$ 


\section{Capítulo 5}

\section{Propriedades magnéticas de nanofios de Mn sobre a superfície $\mathbf{F e}(110)$}

Consideramos fios com até seis átomos de Mn depositados em $\mathrm{Fe}(110)$ e comparamos os resultados com os obtidos para nanofios de $\mathrm{Mn}_{n} / \mathrm{Fe}(001)$.

\subsection{Preâmbulo}

Nossos resultados para nanofios de $\mathrm{Mn}$ em Fe(001) indicam, como mostrado no capítulo anterior, que as interações de troca magnética têm uma forte dependência com o meio local. Vimos também que a competição entre as interações de troca magnética de diferentes vizinhanças e efeitos do acoplamento spin-órbita levam a estruturas magnéticas não colineares, com energias muito próximas as das colineares. É interessante, assim, estudar também o caso de outra direção de superfície e escolhemos aqui a Fe(110). Resultados experimentais para monocamadas de Mn em Fe(110) [47] mostram que para coberturas até 0,3 ML de Mn sobre o $\mathrm{Fe}(110)$ a magnetização resultante é nula, enquanto que no intervalo 0,4-2,0 ML de Mn sobre o Fe(110) a magnetização resultante é de $\approx 1,0 \mu_{B}$. Estes resultados contrastam com os dados 
experimentais para a superfície $\mathrm{Fe}(001)$ já mencionados no capítulo anterior (seção 4.1).

\subsection{Detalhes computacionais}

A superfície de $\mathrm{Fe}(110)$ foi modelada por um cluster contendo 10560 átomos posicionados em uma rede bcc com o parâmetro de rede experimental do Fe bulk $a=2,87 \AA$. O mesmo procedimento utilizado no cálculo de $\mathrm{Fe}(001)$ é também usado para Fe(110) (Seção 4.2). Neste capítulo, não apresentaremos os resultados obtidos para a superfície $\mathrm{Fe}(110)$, uma vez que os mesmos são discutidos em detalhe na Ref. [71]. Consideramos cadeias lineares com até seis átomos de Mn depositados em Fe(110). Os sítios de Mn e seus primeiros vizinhos Fe foram recalculados de forma autoconsistente, com o tamanho desta região variando entre 9 e 32 sítios, sendo que os parâmetros de potencial para os outros sítios, distantes dos átomos de Mn, foram mantidos fixos. A metodologia aqui utilizada no estudo de nanofios de Mn em $\mathrm{Fe}(110)$ é análoga à descrita no capítulo anterior (Seção 4.2).

\subsection{Nanofios de Mn sobre a superfície Fe(110)}

\subsubsection{Estuturas colineares}

Na Fig. 5.1 mostramos as geometrias das várias cadeias lineares finitas de Mn adsorvidas em Fe(110) aqui consideradas. As configurações para os nanofios são rotuladas por $\mathrm{Mn}_{n} / \mathrm{Fe}(110)$. Fizemos cálculos para diferentes configurações magnéticas para os nanofios de Mn. Aqui usaremos as mesmas notações do caso do $\mathrm{Mn}_{n} / \mathrm{Fe}(001)$ (Fig. 4.10). Nestes nanofios de Mn sobre o $\mathrm{Fe}(110)$ a distância entre dois átomos de Mn são de primeiros vizinhos, $\frac{a \sqrt{3}}{2}$, onde cada átomo de Mn terá dois primeiros vizinhos Fe localizados em $\mathrm{Fe}(\mathrm{S})$.

Nossos resultados indicam que o $m_{L}$ é pequeno, sendo $\approx 0,02 \mu_{B} /$ átomo e 0,04 $\mu_{B}$ /átomo para os átomos de Mn e Fe, respectivamente. Sendo assim, 


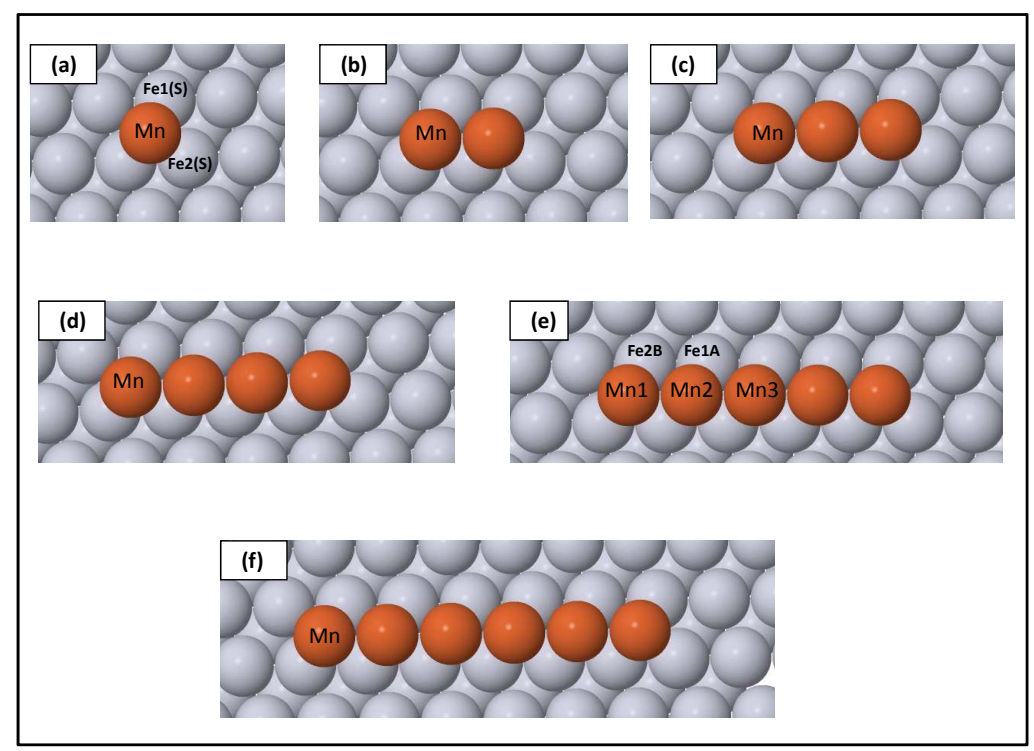

Figura 5.1: Cadeias lineares finitas de átomos de Mn adsorvidas em uma superfície Fe(110): (a) adátomo, (b) dímero, (c) trímero, (d) $\mathrm{Mn}_{4}$, (e) $\mathrm{Mn}_{5}$ e (f) $\mathrm{Mn}_{6}$. As esferas vermelhas e cinzas indicam os átomos de Mn e Fe, respectivamente.

passamos a discutir os resultados de $m_{s}$. Nossos cálculos indicam que as contribuições de spin para os momentos magnéticos do Mn podem variar consideravelmente. Para o caso (i)-(FM) (Fig. 4.10) foram obtidos $m_{s_{M n}}=$ $4,2 \mu_{B}$ para um único átomo de $\mathrm{Mn}$ depositado em $\mathrm{Fe}(110), m_{s_{M n}} \approx 3,8 \mu_{B}$ para átomos de Mn localizados nas extremidades da cadeia, e $m_{s_{M n}} \approx 3,3$ $\mu_{B}$ para sítios de Mn localizados na região interna da cadeia linear. Nossos resultados para sítios de $\mathrm{Mn}$ acoplados $\mathrm{AF}$ à magnetização do substrato são sistematicamente maiores que os obtidos no caso do nanofio de Mn FM, variando de $m_{s_{M n}} \approx 3,9 \mu_{B}$ para átomos de Mn localizados nas extremidades da cadeia até $m_{s_{M n}} \approx 3,5 \mu_{B}$ para átomos de Mn localizados na parte interna da cadeia. Os momentos magnéticos de spin nos átomos de Fe primeiros vizinhos são $\approx 2,0 \mu_{B}$ e não são muito modificados pela quantidade de primeiros vizinhos Mn que o Fe tem. 
Começamos a análise da configuração magnética de um único átomo de Mn adsorvido em Fe(110) [Fig. 5.2(a)] em comparação com a impureza de Mn [Fig. 5.2(b)] embebida na primeira camada de Fe(110). Nossos cálculos indicam que o momento magnético do adátomo Mn acopla-se AF com os momentos magnéticos do Fe, enquanto que o momento magnético da impureza de Mn acopla-se FM. Estes resultados são opostos aos obtidos no caso do adátomo e impureza de $\mathrm{Mn}$ em $\mathrm{Fe}(001)$, onde o adátomo Mn acopla-se FM e a impureza AF. A diferença de energia entre as configurações (i)-(FM) e (ii)-(AF) é $\approx 4 \mathrm{meV} /$ átomo para o adátomo e $\approx 7 \mathrm{meV} /$ átomo para a impureza. Em ambos os casos, o ordenamento magnético é uma conseqüência da interação de troca magnética entre os átomos de Mn e Fe primeiros vizinhos e segundos vizinhos. O valor da interação de troca magnética entre o adátomo Mn e seus dois primeiros vizinhos Fe1(S) [Fig. 5.1-(a)], calculados a partir da configuração FM, é $\mathrm{J}_{M n-F e 1(S)}=-11 \mathrm{meV}$ e o acoplamento entre o Mn e Fe2(S), segundos vizinhos, é $J_{M n-F e 2(S)}=-7 \mathrm{meV}$. O valor de $\mathrm{J}_{i j}$ entre a impureza de Mn e seus seis primeiros vizinhos Fe, localizados na Fe(S-1), é $8 \mathrm{meV}$ e para Fe localizado na mesma superfície da impureza $\mathrm{Fe}(\mathrm{S})$ de Mn é 14 meV. A configuração mais estável (FM) da impureza de Mn tem um momento magnético $m_{s_{M n}}=2,42 \mu_{B}$. Os momentos magnéticos dos primeiros vizinhos Fe na camada $\mathrm{Fe}(\mathrm{S})$ são $m_{s_{F e(S)}}=2,43 \mu_{B}$, e os localizados na subsuperficie $m_{s_{F e(S-1)}}=2,05 \mu_{B}$. Na Fig. 5.3 apresentamos as LDOS para o

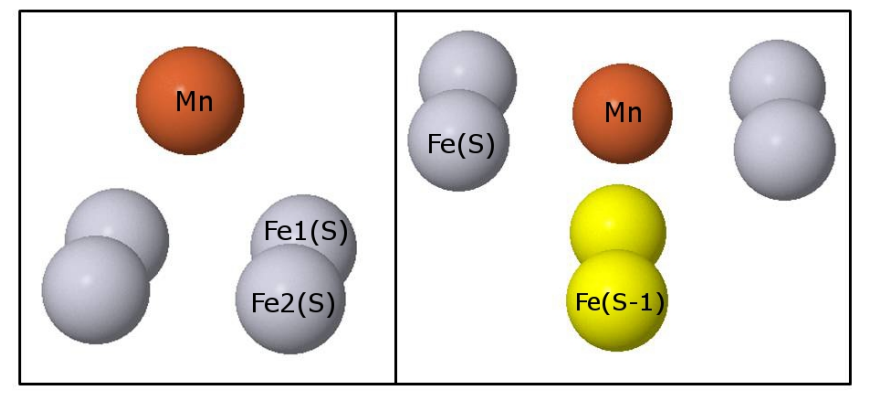

Figura 5.2: Ilustração do (a) adátomo e (b) impureza de Mn sobre o Fe(110). Note-se que existem dois primeiros vizinhos Fe para o adátomo, enquanto a impureza tem seis primeiros vizinhos Fe. 
adátomo e impureza na superfície Fe(110) ambos na configuração FM. Podemos ver os efeitos dos diferentes números de primeiros vizinhos no caso do adátomo [dois primeiros vizinhos Fe - Fig. 5.2(a)] e impureza [seis primeiros vizinhos Fe - Fig. 5.2(b)], levando a um maior $m_{s}$ para o adátomo $\left(4,20 \mu_{B}\right)$, comparado ao momento magnético da impureza $\left(2,42 \mu_{B}\right)$
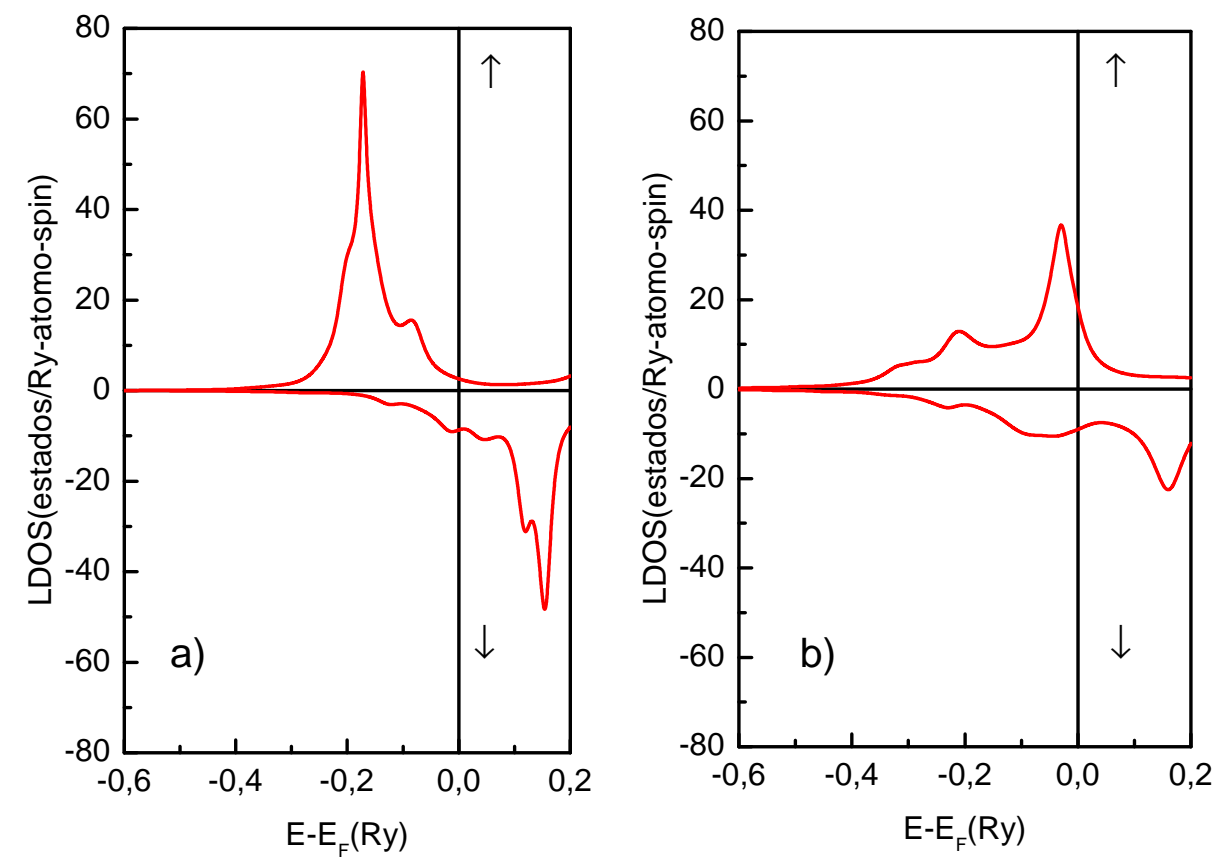

Figura 5.3: Densidade de Estados Local projetado no sítio de (a) adátomo e (b) impureza de Mn em Fe(110).

Para o dímero de Mn adsorvido em Fe(110) [Fig. 5.1(b)], nossos cálculos indicam que a diferença de energia entre as configurações magnéticas (i)(FM) e (iii)-(FI1) são $\approx 21 \mathrm{meV} /$ átomo, em favor da configuração (iii)-(FI1) e as energias (i)-(FM) e (ii)-(AF) são praticamente iguais. Para os nanofios maiores, $\mathrm{n}>3$, obtivemos que fios com números pares de átomos de Mn têm a configuração magnética (iii.1)-(FI-1) com mais baixa energia. As diferenças de energia entre as configurações (i)-(FM) e (iii)-(FI-1) é $\approx 27 \mathrm{meV} /$ átomo e $36 \mathrm{meV} /$ átomo para os nanofios $\mathrm{Mn}_{4} / \mathrm{Fe}(110)$ e $\mathrm{Mn}_{6} / \mathrm{Fe}(110)$, respectivamente. Para as cadeias com números ímpares de átomos de Mn a con- 
figuração magnética estável é a configuração (iii.2)-(FI-2). As diferenças de energia entre (iii.2)-(FI-2) e (i)-(FM) são sempre maiores que 25 meV/átomo. Estes resultados apresentam diferenças em energia maiores que as calculadas no caso de nanofios de Mn depositados em uma superfície Fe(001), onde várias configurações magnéticas colineares apresentam estados estáveis ou metaestáveis. Contudo, a diferença entre as configurações (iii.2)-(FI-2) e (iii)-(FI-3) são relativamente pequenas ( $<9 \mathrm{meV} /$ átomo) para cadeias com números impares de Mn.

A fim de obter uma melhor interpretação destes resultados, mostramos na Fig. 5.4-(a) os valores calculados da interação de troca magnética entre os momentos magnéticos do $\mathrm{Mn}\left(\mathrm{J}_{M n-M n}\right)$ e entre os momentos magnéticos dos átomos de $\mathrm{Mn}$ e Fe $\left(\mathrm{J}_{M n-F e}\right)$ na Fig. 5.4-(b) para todas as cadeias lineares de Mn. Todos os valores foram obtidos a partir da configuração FM. A magnitude das interações de troca Mn e Fe é menor do que a magnitude de $J_{M n-M n}$ de primeiros vizinhos. Podemos ver na Fig. 5.4-(a) que $\mathrm{J}_{M n-M n}$ para Mn-Mn primeiros vizinhos é forte e negativa, decrescendo em módulo para os próximos vizinhos $\mathrm{Mn}$, oscilando entre valores negativos e positivos para maiores distâncias. Podemos também notar na Fig.5.4 (b) que $\mathrm{J}_{M n-F e}$ tem valores altos para separações interatômicas além de primeiros vizinhos. Por exemplo, para $\mathrm{Mn}_{5} / \mathrm{Fe}(110)$ [Fig. 5.1-(e)] a interação de troca magnética para primeiros vizinhos $\mathrm{Mn}-\mathrm{Fe}$ é $\mathrm{J}_{M n 2-F e 1 A}=-11 \mathrm{meV}$ e $\mathrm{J}_{M n 2-F e 1 B}=14$ meV para segundos vizinhos $\mathrm{Mn}-\mathrm{Fe}$. Os valores de $J_{M n-F e}$ oscilam entre valores negativos e positivos para maiores distâncias, sendo uma oscilação do tipo RKKY [79-81]. Análogo do caso de $\mathrm{Mn}_{n} / \mathrm{Fe}(001)$ notamos que para $\mathrm{Mn}_{n} / \mathrm{Fe}(001), \mathrm{J}_{M n-M n}$ depende do número de átomos Mn que são primeiros vizinhos. Também calculamos as interações $\mathrm{J}_{\mathrm{Fe}-\mathrm{Fe}}$ e verificamos que os valores entre primeiros vizinhos são positivos e decrescem rapidamente para vizinhos mais distantes. 

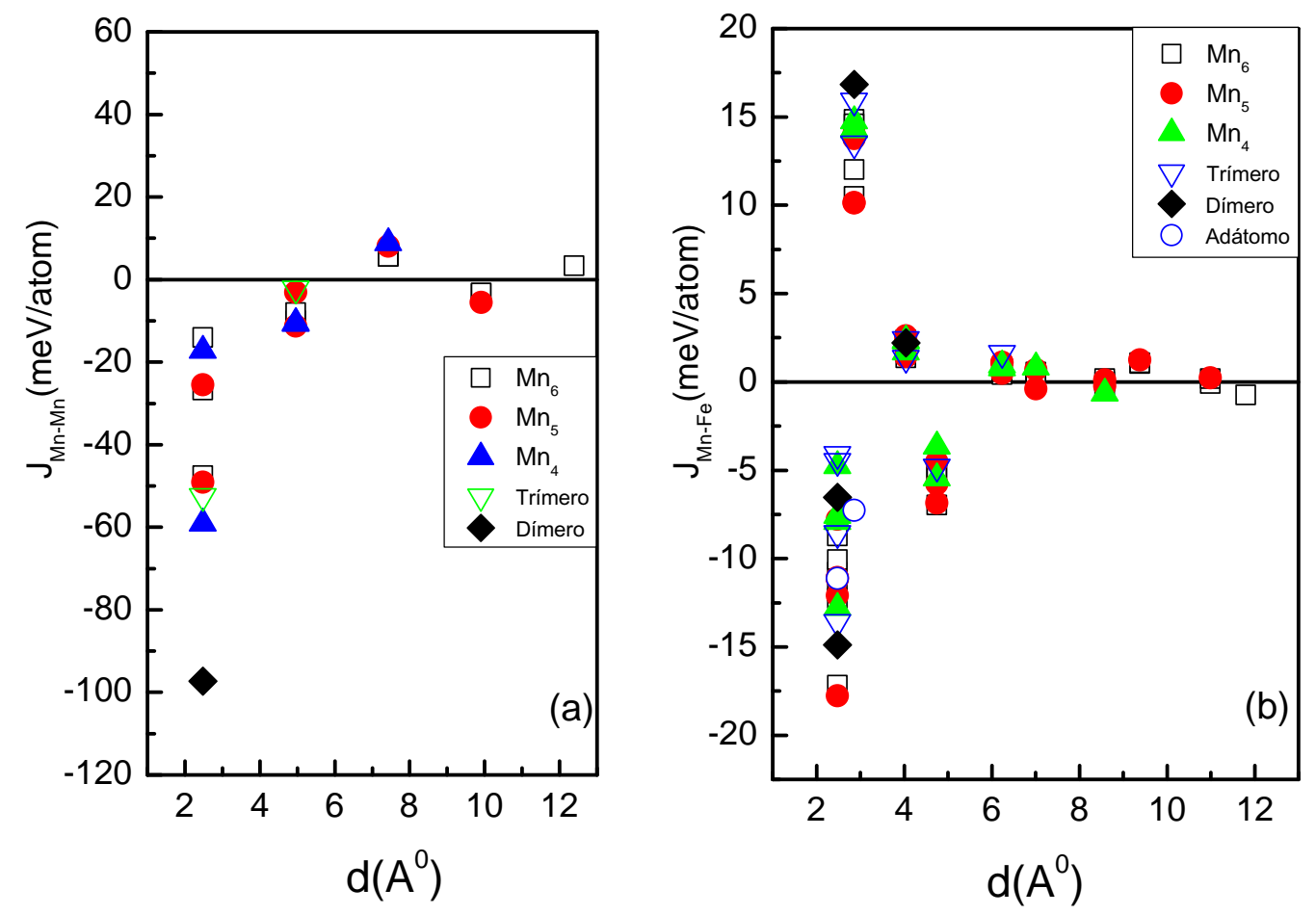

Figura 5.4: Interações de troca magnética em função da distância entre (a) átomos de $\mathrm{Mn}, \mathrm{J}_{M n-M n}$, e (b) átomos de $\mathrm{Mn}$ e Fe, $\mathrm{J}_{M n-F e}$.

\subsubsection{Resultados não colineares}

A competição entre interações de troca magnética entre pares Mn-Mn e Mn-Fe sugere que as configurações magnéticas de nanofios de Mn adsorvidas em Fe(110) podem ser não colineares. Exploramos esta possibilidade fazendo cálculos não colineares para os nanofios de Mn apresentados nas Figs. 5.1(b)5.1(f), considerando a interação spin-órbita. Nossos resultados são mostrados nas Figs. 5.5(a)-5.5(e). Iniciamos a análise da não colinearidade para o dímero de $\mathrm{Mn}$, onde o ângulo entre os momentos magnéticos do Mn-Mn é $113^{0}$ e Mn-Fe fica em torno de $154^{0}$. Esta configuração magnética deve-se a competição entre a interação AF entre os átomos de $\mathrm{Mn}\left(J_{M n-M n}=-98 \mathrm{meV}\right)$ e entre Mn com os seus primeiros vizinhos $\left(J_{M n-M n}=-11 \mathrm{meV}\right)$. Para os fios maiores, n>3, Figs. 5.1(b)-(f) as configurações magnéticas não colineares 


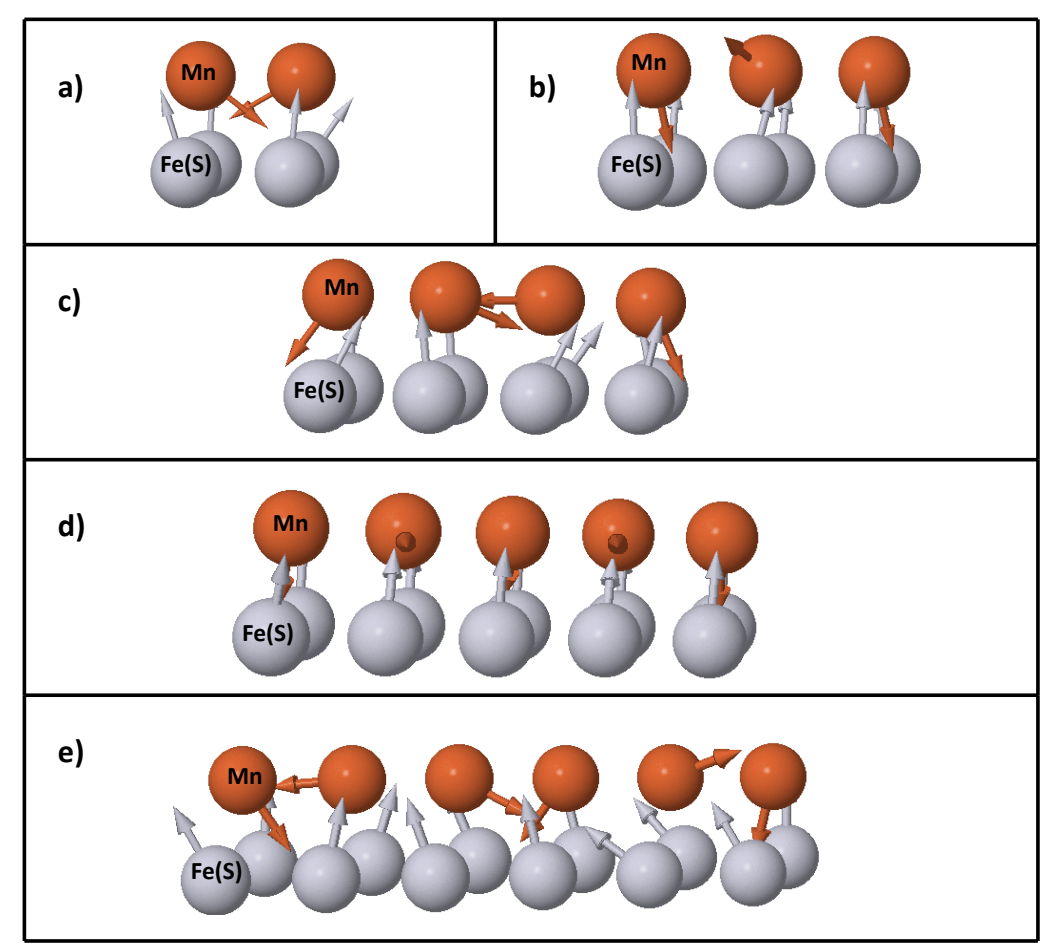

Figura 5.5: Configurações magnéticas não colineares para nanofios com (a) $\mathrm{Mn}_{2}$, (b) $\mathrm{Mn}_{3}$, (c) $\mathrm{Mn}_{4}$, (d) $\mathrm{Mn}_{5}$, and (e) $\mathrm{Mn}_{6}$ em Fe(110).

podem ser vistas da seguinte forma: os momentos magnéticos dos átomos de Mn na extremidade do nanofio são aproximadamente AF com os momentos dos átomos de Fe primeiros vizinhos. Também a direção dos momentos magnéticos entre átomos de Mn primeiros vizinhos são aproximadamente perpendiculares. Para os momentos magnéticos dos átomos de Mn na parte interna da cadeia, temos que estes são aproximadamente perpendiculares a magnetização dos sítios do Fe.

Fizemos também uma análise energética para os nanofios $\mathrm{Mn}_{n} / \mathrm{Fe}(110)$. A diferença de energia nos casos não colineares e colineares de menor energia são $90 \mathrm{meV} /$ átomo e $122 \mathrm{meV} /$ átomo, em favor para o dímero e trímero colinear, respectivamente. Para os nanofios $\geq 4$, as configurações magnéticas não colineares [Figs. 5.5(c)-5.5(e)] são as soluções mais estáveis. Também fizemos cálculos sem o acoplamento spin-órbita para o fio $\mathrm{Mn}_{6} / \mathrm{Fe}(110)$ e 
nossos resultados indicam que não existem diferenças significativas entre as configurações magnéticas sem o acoplamento e com acoplamento spin-órbita. Estes resultados diferem dos obtidos para os fios longos de Mn em Fe(001), onde existem contribuições não desprezíveis das interações de DzyaloshinskiiMoriya. Estas características magnéticas diferentes apresentadas por cadeias de Mn em uma superfície Fe(110) e Fe(001) bcc surgem das diferentes competições entre as interações de troca magnética destes dois sistemas. 


\section{Capítulo 6}

\section{Propriedades magnéticas em cadeias lineares de $\mathrm{Fe}_{x} \mathrm{Co}_{1-x}$ depositadas sobre $\operatorname{Pt}(111)$}

Neste capítulo, apresentamos as propriedades eletrônicas e magnéticas de nanofios de $\mathrm{Fe}_{x} \mathrm{Co}_{1-x}$ depositados sobre uma superfície $\mathrm{Pt}(111)$. O estudo deste sistema se deve ao interesse em aplicações tecnológicas de materiais que possam ser usados em dispositivos de gravação magnética. Consideramos fios lineares com até nove átomos, onde foram levados em conta todos os arranjos possíveis de Fe e Co.

\subsection{Preâmbulo}

Conforme mencionado anteriormente, uma das principais motivações de pesquisa em magnetismo são as aplicações tecnológicas em sistemas de gravação magnética. O princípio fundamental é que a informação é decomposta em unidades binárias (bits) para o armazenamento de dados magnéticos, onde cada bit é armazenado na direção de magnetização do meio magnético. Devido ao interesse no aumento da capacidade de armazenamento de dados, estes bits devem apresentar a maior densidade possível (números de bits por 
unidade de área) [126].

Existem três partes dos dispositivos de gravação magnética que podem ser identificados como importantes para a densidade de armazenamento de dados (Fig. 6.1) [77]. O primeiro é chamado de read head que lê a informação a partir do meio magnético. Tipicamente o read head é um sensor magnético construído por um sanduíche de camadas magnéticas e não magnéticas que pode usar os efeitos de magnetorresistência gigante (GMR) [1] ou efeitos de magnetorresistência de tunelamento (TMR) [127]. Outra parte do dispositivo

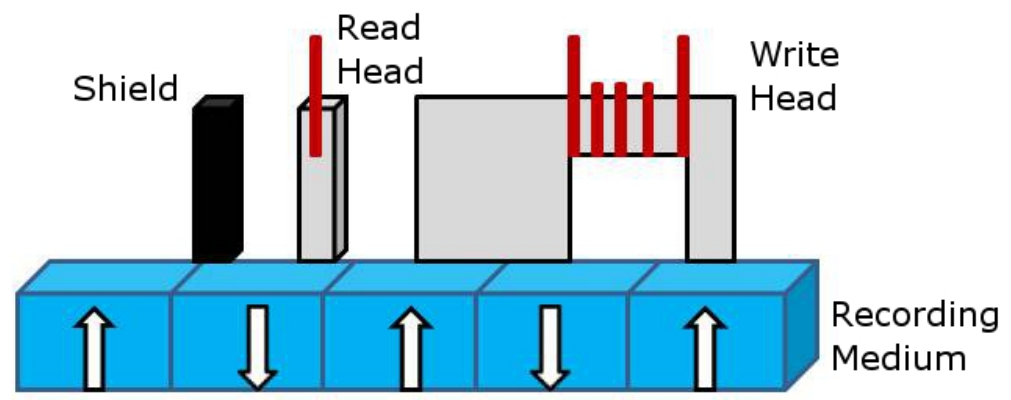

Figura 6.1: Ilustração das três partes de um dispositivo de gravação magnética.

é o chamado meio de gravação magnética (recording medium). Aqui, muitas propriedades são necessárias, para permitir que a informação seja escrita no meio de gravação a altas velocidades e também que a informação gravada seja mantida inalterada por muito tempo. Uma propriedade importante é que o meio magnético deve possuir uma alta energia de anisotropia magneto cristalina $(M A E)$, para manter o meio de gravação estável contra flutuações térmicas e também para evitar interações entre bits vizinhos.

Finalmente a write head, que é o dispositivo responsável por escrever a informação no meio magnético, necessariamente esta parte deve produzir um campo magnético suficientemente forte para girar (flip) a direção magnética dos bits, também deve ser localizada para evitar perturbar os bits vizinhos. Assim, para que o campo magnético do write head aumente, é necessário aumentar a sua densidade de magnetização. A densidade de magnetização 
de um material vai depender do seu momento magnético. Neste contexto, como foi dito no Capítulo 1, a liga bcc FeCo é um promissor sistema para a write head devido ao fato de que resultados tanto experimentais [48] quanto teóricos $[49,50]$ indicam que o $m_{s}$ médio da liga segue a curva de SlaterPauling, com um máximo em torno de $2,4 \mu_{B}$ em $\approx 30 \%$ de Co [51]. Este fato pode ser entendido através da densidade de estados local para o Fe bulk, sendo este um ferromagneto fraco, com o nível de Fermi cortando as bandas $3 \mathrm{~d} \uparrow$ e $3 \mathrm{~d} \downarrow$, enquanto que o Co é um ferromagneto forte com a banda majoritária completamente ocupada. A hibridização entre Fe e o Co produz um aumento do exchange splitting do Fe puro, correspondendo a um aumento do número de elétrons na banda majoritária em comparação à banda minoritária, aumentando o $m_{s}$ do Fe. O aumento de elétrons na banda majoritária do Fe se origina da banda minoritária do Co, sendo que o número de elétrons da banda majoritária do Co permanece constante. Este efeito alcança um máximo em aproximadamente $30 \%$ de Co; valor a partir do qual o número de elétrons na banda majoritária do Fe permanece constante, enquanto o número de elétrons na banda minoritária aumenta, diminuindo o $m_{s}$. O $m_{L}$ da liga FeCo varia quase que linearmente com a concentração de Co [50]. Um ponto importante é que este sistema tem uma MAE pequena, devido à simetria cúbica da liga FeCo.

O desafio, atualmente, é construir sistemas artificiais de FeCo que possuam momentos magnéticos que superem os valores do bulk bcc. Uma maneira é construir estruturas, por exemplo, com distorções de rede [52], multicamadas [53-55] e aglomerados depositados em superfícies [56]. Neste contexto, resultados teóricos indicam que em ligas FeCo quando submetidos a distorções específicas, por exemplo, estrutura tetragonal de corpo centrado, a $M A E$ aumenta consideravelmente $(0,8 \mathrm{meV})$ [52]. O momento magnético é da ordem de 2,1 $\mu_{B}$ para a concentração onde a $M A E$ tem um máximo. Para efeito de comparação, citamos a $M A E$ do Fe bcc e Co hexagonal compacta $h c p$ são $5 \mu \mathrm{eV}$ e $45 \mu \mathrm{eV}$, respectivamente. Para filmes de FeCo cálculos dentro da DFT indicam altos valores de momento magnético e $M A E$ (comparável aos da Ref. [52] ) quando o parâmetro de rede no plano e fora do plano são modi- 
ficados [54]. Resultados experimentais para filmes de $\mathrm{Fe}_{x} \mathrm{Co}_{1-x}$ depositados em uma superfície $\mathrm{Rh}(001)$ indicam uma alta MAE perpendicular ao plano que fica em torno de 0,6 meV, para uma concentração de $60 \%$ de Co, onde o $m_{L}$ do Co tem uma dependência em função da composição química similar à $M A E$ com um valor máximo de $0,32 \mu_{B}$ [55]. Outro trabalho interessante da literatura mostra que resultados experimentais e teóricos para uma monocamada de FeCo depositada sobre uma superfície de Pt(111) indicam altos valores de momento magnético e MAE [53] em comparação com o bulk bcc FeCo: a $M A E$ do filme de FeCo é mais que 2 ordens de magnitude maior que os valores observados para a liga $b c c \mathrm{Fe}_{0,5} \mathrm{Co}_{0,5}$. Os valores teóricos do $m_{s} \mathrm{e}$ $m_{L}$ para a concentração onde a $M A E$ tem um máximo são $\approx 2,6 \mu_{B} \mathrm{e} \approx 0,15$ $\mu_{B}$, respectivamente.

Motivados por estas discussões, realizamos cálculos de primeiros princípios no estudo das propriedades magnéticas de cadeias lineares de $\mathrm{Fe}_{x} \mathrm{Co}_{1-x}$ depositadas em uma superfície $\mathrm{Pt}(111)$. A diferença entre os nanofios de $\mathrm{Fe}_{x} \mathrm{Co}_{1-x}$ e os resultados experimentais e teóricos de filmes de $\mathrm{Fe}_{x} \mathrm{Co}_{1-x}$ depositados sobre uma superfície $\operatorname{Pt(111)~[53]~são~também~discutidos~neste~}$ capítulo.

\subsection{Detalhes Computacionais}

Consideramos nanofios $\mathrm{Fe}_{x} \mathrm{Co}_{1-x}$ com diferentes concentrações de $\mathrm{Fe}$, denotado por $x$, depositados em uma superfície Pt(111). A superfície Pt(111) foi modelada por um cluster contendo 5000 átomos posicionados em uma rede cúbica de face centrada $f c c$ com o parâmetro de rede experimental da Pt, $a=3,92 \AA$. O mesmo procedimento descrito no cálculo de Fe(001) (Seção 4.2) foi também usado para $\operatorname{Pt(111).~Neste~capítulo~não~apresentaremos~os~}$ resultados da superfície $\mathrm{Pt}(111)$, uma vez que os mesmos são discutidos em detalhe nas Refs. [128-130].

Os nanofios de $\mathrm{Fe}_{x} \mathrm{Co}_{1-x}$ foram adsorvidos como uma perturbação da superfície Pt(111) convergida. Foram considerados que os átomos de Fe e Co ocupam posições não relaxadas, em uma rede ideal fcc, assumindo o 
parâmetro de rede da Pt. Os sítios de Fe e Co e também seus primeiros vizinhos $\mathrm{Pt}$ foram recalculados autoconsistentemente, sendo que os parâmetros de potencial para os outros sítios, distantes dos átomos de Fe e Co, foram mantidos fixos. Para cada concentração $x$ (que fixa o número total de átomos de $\mathrm{Fe}$ ), consideramos sempre fios contendo nove átomos e variamos a posição dos Fe, sendo que todos os possíveis arranjos foram levados em consideração. Na Fig.6.2 apresentamos uma ilustração para o caso $\mathrm{Fe}_{0,11} \mathrm{Co}_{0,89}$.

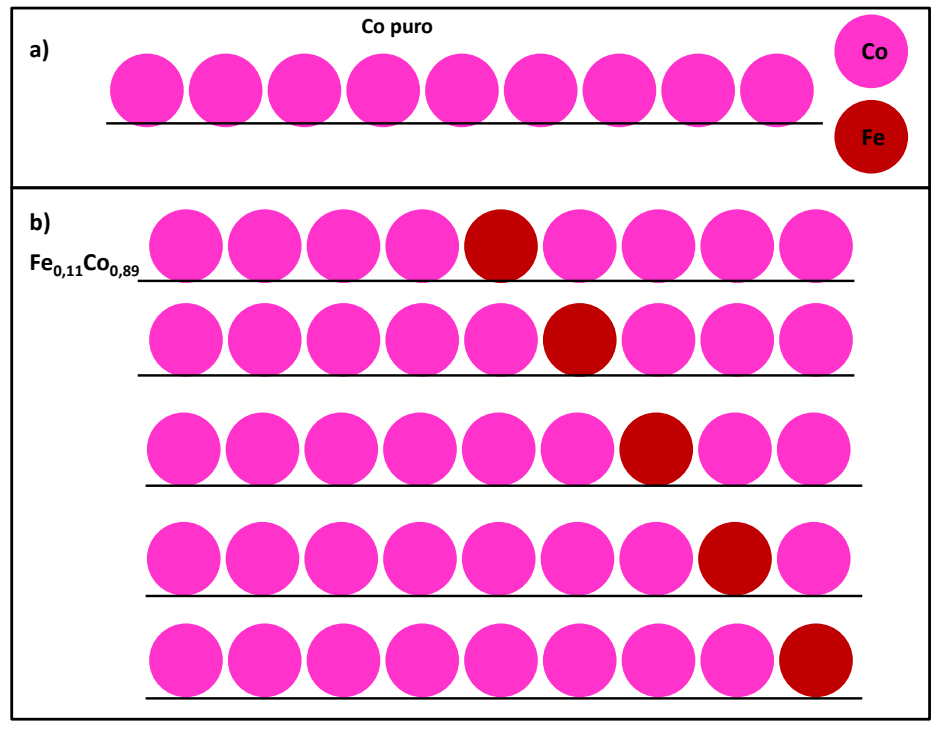

Figura 6.2: Ilustração do nanofio de (a) Co puro e (b) $\mathrm{Fe}_{0,11} \mathrm{Co}_{0,89}$ depositados em $\mathrm{Pt}(111)$, considerando os arranjos possíveis neste caso.

A interação de troca de Heisenberg $J_{i j}$ entre os momentos magnéticos dos átomos foi calculada pela [Eq. (3.3)] e para analisar o acoplamento magnético entre os átomos do nanofio de $\mathrm{Fe}_{x} \mathrm{Co}_{1-x}$ depositados na superfície $\mathrm{Pt}(111)$, consideramos que o $m_{s}$ dos átomos de Fe e Co são acoplados FM. Foi considerada a inclusão da polarização orbital de Brooks (OP) [63] para a correção do valor de $m_{L}$ como implementado no RS-LMTO-ASA [131]. 


\subsection{Resultados e Discussões}

\subsubsection{Momentos magnéticos}

Na Fig. 6.3 mostramos algumas cadeias lineares finita de $\mathrm{Fe}_{x} \mathrm{Co}_{1-x}$ sobre uma superfície $\operatorname{Pt}(111)$ para três valores de $x: 1,0 ; 0,56$ e 0,22. Nestes casos, cada átomo de Fe ou Co possuim três primeiros vizinhos Pt e também dois primeiros vizinhos $\mathrm{Fe}$ ou Co, quando o Fe ou Co estão localizados no interior do nanofio, enquanto átomos de $\mathrm{Fe}$ ou Co localizados na extremidade da cadeia possuem um primeiro vizinho Fe ou Co.

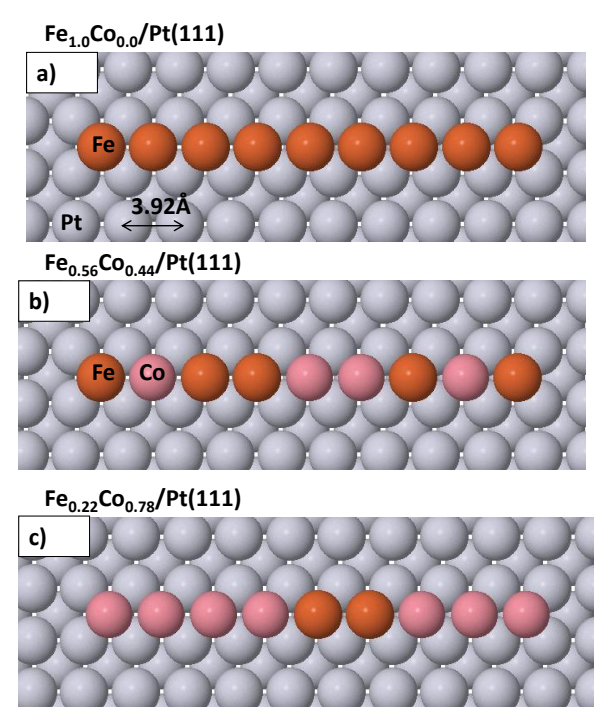

Figura 6.3: Cadeias lineares finitas de $\mathrm{Fe}_{x} \mathrm{Co}_{1-x}$ adsorvidas em uma superfície $\operatorname{Pt}(111)$ : a) $\mathrm{Fe}_{1,0} \mathrm{Co}_{0,0}$, b) $\mathrm{Fe}_{0,56} \mathrm{Co}_{0,44}$ e c) $\mathrm{Fe}_{0,22} \mathrm{Co}_{0,78}$. As esferas vermelhas, rosas e cinzas indicam os átomos de $\mathrm{Fe}$, Co e Pt, respectivamente.

Começamos pela análise dos valores médios do $m_{s}$ e $m_{L}$ da liga em função de $x$. Para os cálculos de valores médios de momentos magnéticos médios foram usadas várias configurações para um dado valor de $x$. Os valores médios dos momentos magnéticos (spin e orbital) da liga, de Fe e Co, foram determinados através da seguinte relação:

$$
\bar{m}=x m_{F e}+(1-x) m_{C o} .
$$


Na Fig. 6.4 apresentamos os valores de $\bar{m}_{s}, \bar{m}_{L}, m_{s}$ e $m_{L}$ (médias dos momentos magnéticos do $\mathrm{Fe}$ e Co) obtidos nos nanofios de $\mathrm{Fe}_{x} \mathrm{Co}_{1-x}$ sobre $\mathrm{Pt}(111)$. Observamos inicialmente que os $m_{s}$ do Fe e do Co são fracamente dependentes de $x$. O que resulta no valor médio de $m_{s}$ que é uma função linear da concentração de Fe; resultado este que é diferente quando comparado a curva de Slater-Pauling. Contudo, esta tendência do momento magnético médio apresentada na Fig. 6.4(a) foi também reportada na literatura para a monocamada de $\mathrm{Fe}_{x} \mathrm{Co}_{1-x}$ depositada sobre $\mathrm{Pt}(111)$ [53], que doravante denotaremos por FeCo/Pt(111). Entretanto, os $m_{s}$ do Fe e do Co aumentam quando comparados aos do FeCo/Pt(111) e o bulk: os valores médios de $m_{s}$ do Fe são aproximadamente $3,5 \mu_{B}$ (sendo $3,0 \mu_{B}$ para a superfície e $2,2 \mu_{B}$ para o bulk) e do Co aproximadamente $2,2 \mu_{B}$ (sendo $2,0 \mu_{B}$ para a superfície e $1,6 \mu_{B}$ para o bulk). Este aumento, como seria esperado, devido à redução da dimensionalidade e coordenação atômica.

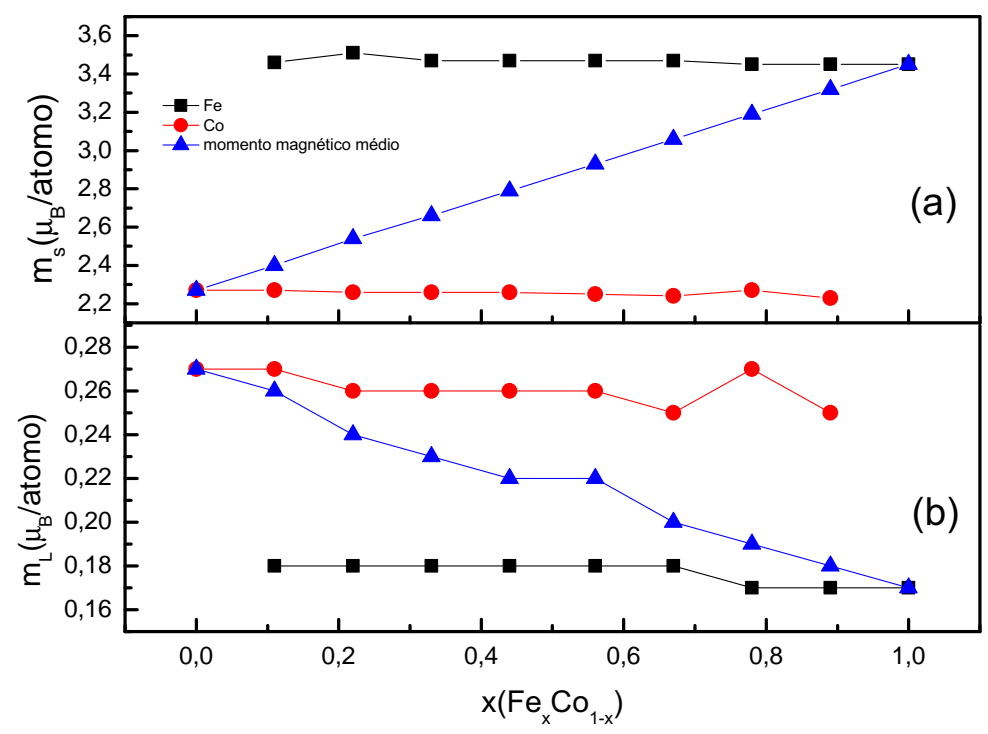

Figura 6.4: Comportamento dos momentos magnéticos $(\mathrm{a}) \mathrm{m}_{s}$ e (b) $\mathrm{m}_{L}$ dos átomos de $\mathrm{Fe}$ e Co em nanofios de $\mathrm{Fe}_{x} \mathrm{Co}_{1-x}$ sobre superfície $\mathrm{Pt}(111)$. 
Na Fig. 6.4(b) apresentamos os resultados para $m_{L}$ e verificamos que $m_{L}$ médio é monotonicamente decrescente com $x$. Nossos resultados indicam que o $m_{L}$ do Fe tem uma fraca dependência com $x$ e para o Co o $m_{L}$ tem uma um valor máximo de $0,27 \mu_{B}$ que ocorre em $\mathrm{x}=0 ; 0,11$ e 0,78 . Os valores de $m_{L}$ médio para o Fe ficam em torno de $0,18 \mu_{B}$ (sendo $0,13 \mu_{B}$ para a superfície e $0,043 \mu_{B}$ para o bulk) e para o Co aproximadamente $0,26 \mu_{B}$ (sendo $0,16 \mu_{B}$ para a superfície e $0,078 \mu_{B}$ para o bulk). Este resultado é diferente do que foi observado para o $\mathrm{FeCo} / \mathrm{Pt}(111)$, onde foi calculado uma forte dependência de $m_{L}$ médio com a composição da monocamada, tendo um máximo para $60 \%$ de Co. Entretanto, o $m_{L}$ médio obtido em nosso trabalho também foi observado na Ref. [56] para alguns aglomerados de FeCo depositados em $\mathrm{Cu}(100)$. Adicionalmente, o $m_{L}$ da liga bulk bec FeCo varia quase que linearmente com a concentração de Co [50].

Os momentos magnéticos totais induzidos nos átomos de $\mathrm{Pt}$, que são primeiros vizinhos dos átomos de Fe e Co, também foram calculados e são apresentados na Fig. 6.5 e variam de $0,19 \mu_{B}$ para o nanofio de Fe puro até 0,18 $\mu_{B}$ para o Co puro. Este comportamento é diferente do que foi obtido para $\mathrm{FeCo} / \mathrm{Pt}(111)$ [53], onde o momento magnético total para os átomos de Pt da primeira camada da superfície variam de $0,23 \mu_{B}$ para a camada de Fe puro até $0,28 \mu_{B}$ para a camada de Co. Entretanto, através dos nossos cálculos, verificamos que o $m_{s}$ e $m_{L}$ dos átomos de Pt são colineares com a magnetização do nanofio, fato que concorda com os resultados obtidos no caso FeCo/Pt(111) [53]. Além disso, verificamos que os momentos magnéticos dos átomos de Pt da primeira camada, que estão a distâncias maiores que as de primeiros vizinhos, ficam em torno de $0,05 \mu_{B}$.

\subsubsection{Interação de troca magnética}

A fim de um melhor entendimento do acoplamento FM destes nanofios, calculamos os valores das interações de troca magnética entre os momentos magnéticos dos átomos de Fe-Fe, Fe-Co, Fe-Pt, Co-Co e Co-Pt para alguns valores de $x$. No caso do nanofio de Fe puro sobre $\mathrm{Pt}(111)$, verificamos que a 


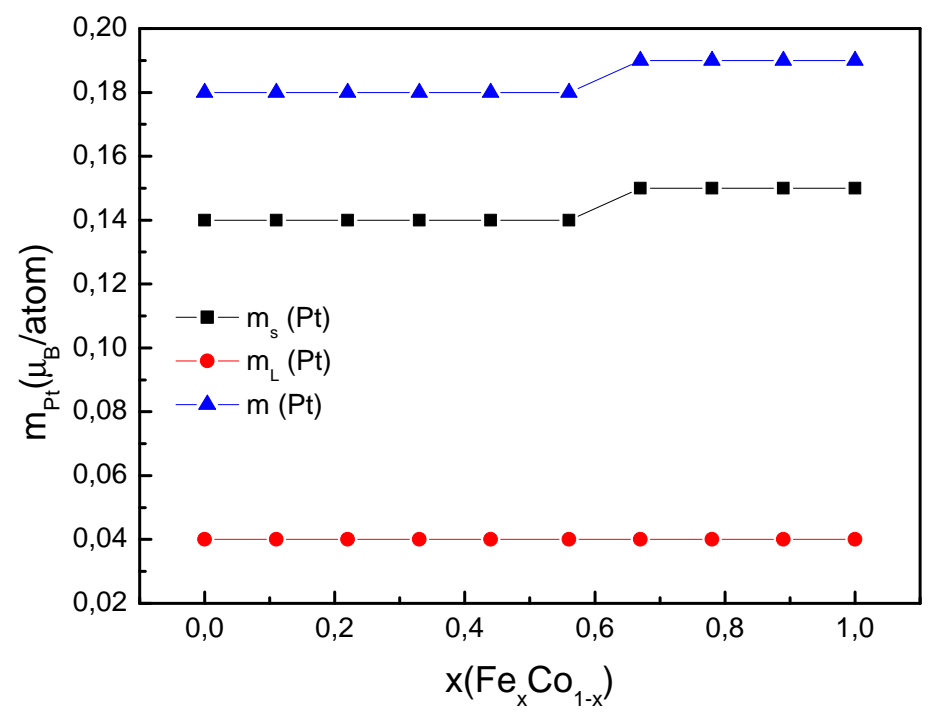

Figura 6.5: Comportamento do momento magnético dos primeiros vizinhos Pt dos nanofios de $\mathrm{Fe}_{x} \mathrm{Co}_{1-x}$ sobre a superfície $\mathrm{Pt}(111)$ em função de $x$.

interação de troca magnética entre primeiros vizinhos Fe é sempre fortemente positiva $\mathrm{J}_{\mathrm{Fe}-\mathrm{Fe}} \approx 38 \mathrm{meV}$ e decresce rapidamente para os segundos vizinhos $\left(\mathrm{J}_{\mathrm{Fe}-\mathrm{Fe}} \approx 0,7 \mathrm{meV}\right)$. Para a interação de troca magnética entre primeiros vizinhos Fe-Pt, obtivemos através dos nossos cálculos $\mathrm{J}_{F e-P t} \approx 1,4 \mathrm{meV}$. Estes resultados indicam que os momentos magnéticos dos átomos de Fe são acoplados FM no caso da cadeia linear em uma superfície Pt(111). Nossos resultados de $J_{i j}$ para o nanofio de Fe puro são interessantes, pois foi observado para uma monocamada de Fe sobre $\operatorname{Pt}(111)$ um $m_{s}$ efetivo de $\approx 1,2 \mu_{B}$ [53]. Os autores concluíram que este valor de momento magnético para a camada de Fe é provavelmente uma conseqüência de uma estrutura magnética complexa com um ordenamento AFM, como observado para a monocamada de Fe em $\operatorname{Ir}(111)$ [123]. É conhecido da literatura [132,133] o Fe fcc apresenta um magnetismo não colinear fortemente dependente do parâmetro de rede.

Para o nanofio de Co puro, verificamos que a interação de troca magnética entre primeiros vizinhos Co-Co é $\mathrm{J}_{C o-C o} \approx 30 \mathrm{meV}$, enquanto que para vizinhos mais distantes a interação de troca magnética decresce. A interação de 
troca magnética Co-Pt também é positiva, tendo como valor $\mathrm{J}_{C o-P t} \approx 1,4$ meV. Quando consideramos uma concentração intermediária, por exemplo, $\mathrm{x}=0,56, \mathrm{~J}_{\mathrm{Fe}-\mathrm{Co}} \approx 35 \mathrm{meV}$ para primeiros vizinhos e decresce rapidamente para as interações entre vizinhos mais distantes. Verificamos também que o acoplamento de troca magnética entre átomos de Pt é pequena e positiva $\left(\mathrm{J}_{P t-P t} \approx 0,07 \mathrm{meV}\right)$. Diferente do observado nas situações apresentadas no Capítulo 4, onde as interações de troca magnética para nanofios de $\mathrm{Mn}_{n} / \mathrm{Fe}(001)$ são de longo alcance e dependente do meio local, nossos resultados para nanofios de $\mathrm{Fe}_{x} \mathrm{Co}_{1-x}$ sobre $\mathrm{Pt}(111)$ sugerem que o modelo de Heisenberg, que leva em conta apenas as interações entre primeiros vizinhos, é claramente apropriado para descrever estes sistemas.

\subsubsection{Contribuição da polarização orbital}

A inclusão da polarização orbital foi usada, pois a LSDA tipicamente subestima os valores de $m_{L}$ para os metais de transição 3 d. Nossos resultados, apresentados na Fig. 6.6, indicam que os átomos de Co são mais sensíveis à inclusão de $O P$ que os de Fe. Entretanto, a mesma tendência para o $m_{L}$ médio nos casos OP e sem OP foi obtida. Contudo, com a inclusão de $O P$ estes valores são mais que $50 \%$ maiores que os valores sem $O P$. Por exemplo, para o nanofio $\mathrm{Fe}_{0,0} \mathrm{Co}_{1,0}$ foi calculado um valor de $m_{L}$ de $0,27 \mu_{B}$ sem $O P$ e 0,65 $\mu_{B}$ com $O P$. Uma média simples destes valores fornece um $m_{L}$ de $0,46 \mu_{B}$. Neste contexto, para uma monocamada de Co em Pt(111) foi encontrado um magnético orbital de $0,31 \mu_{B}$ [53], isto é, nosso resultado de $m_{L}$ está em acordo com a possibilidade do aumento do momento magnético orbital quando as dimensões diminuem [134]. Os valores de $m_{s}$ do Fe, Co e Pt e os de $m_{L}$ da Pt não são muito sensíveis à inclusão de $O P$ e, desta forma, não são mostrados neste trabalho. 


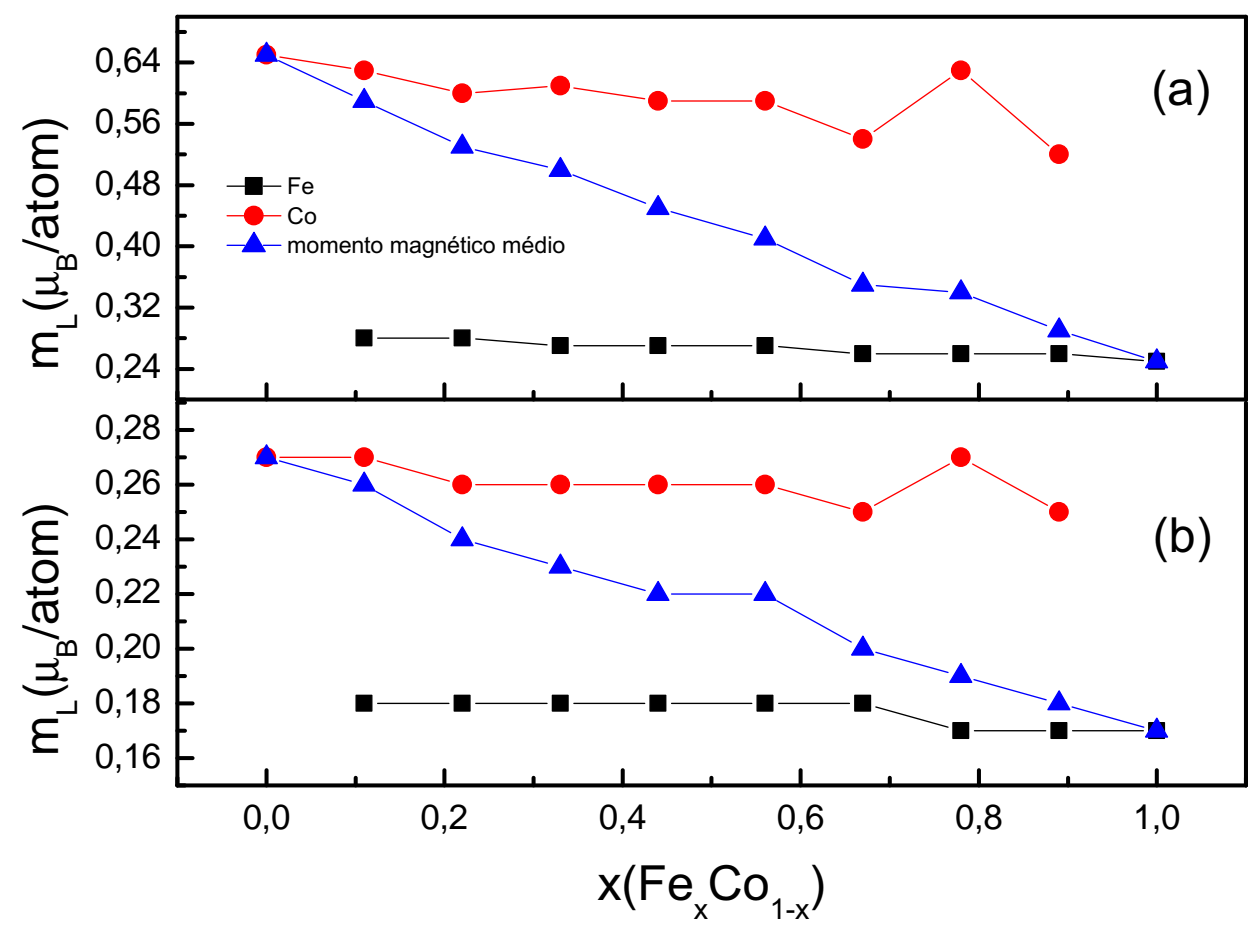

Figura 6.6: Comportamento dos momentos magnéticos orbitais em função de $x$ para nanofios de $\mathrm{Fe}_{x} \mathrm{Co}_{1-x}$ depositados sobre $\mathrm{Pt}(111)$ : a) com OP e b) sem OP.

\subsubsection{Análise de estrutura eletrônica}

Para entender melhor os efeitos de hibridização Fe-Co analisamos as propriedades magnéticas locais destes nanofios, isto é, investigamos como as quantidades e o tipo de vizinhos, que cada átomo de $\mathrm{Fe}(\mathrm{Co})$ tem, influenciam nos seus valores individuais de momentos magnéticos. Consideramos os seguintes casos para a primeira vinhança $(N N)$ : (i) Fe(Co) tem dois $N N$ e (ii) $\mathrm{Fe}(\mathrm{Co})$ tem um NN. Para o primeiro caso, consideramos as seguintes situações:

- (1) $\mathrm{Fe}(\mathrm{Co})$ com dois $N N \mathrm{Fe}(\mathrm{Co})$; 
- (2) $\mathrm{Fe}(\mathrm{Co})$ com um $N N \mathrm{Fe}(\mathrm{Co})$ e um $N N \mathrm{Co}(\mathrm{Fe})$;

- (3) $\mathrm{Fe}(\mathrm{Co})$ com dois $N N \mathrm{Co}(\mathrm{Fe})$.

No caso (1) os $m_{s}$ individuais dos átomos de Fe são $3,4 \mu_{B}$ sendo a única exceção quando o Fe tem dois primeiros vizinhos de Co, onde o valor é 3,5 $\mu_{B}$. Os $m_{s}$ dos átomos de Co são $2,2 \mu_{B}$, independente da vizinhança. Apresentamos na Fig.6.7(a)-(b) as densidades de estados local do Fe(Co), localizados na parte interna da cadeia linear, em função dos diferentes tipos de vizinhos. Verificamos que a banda $3 \mathrm{~d} \uparrow$ do Fe e Co são totalmente preenchidas independentemente do tipo de vizinhança, isto é, ambos são ferromagnetos fortes No caso (ii) existem duas situações:

- (I) $\mathrm{Fe}(\mathrm{Co})$ com um $N N \mathrm{Fe}(\mathrm{Co})$;

- II) $\mathrm{Fe}(\mathrm{Co})$ com um $N N \mathrm{Co}(\mathrm{Fe})$.

Nossos resultados indicam que, para ambas as situações os valores de $m_{s}$ para o $\mathrm{Fe}(\mathrm{Co})$ são independentes do tipo de vizinhança, devido ao preenchimento da banda majoritária (Fig. 6.7(c)-(d) - átomos localizados na extremidade da cadeia linear). Os átomos de Fe e Co têm valores de $m_{s}$ de $3,6 \mu_{B}$ e 2,4 $\mu_{B}$, respectivamente.

Nossos resultados mostram assim que $m_{s}$ do Fe e do Co são independentes da concentração, devido ao preenchimento da banda majoritária, implicando em que a magnetização da liga varia linearmente com a concentração de Fe como mostrado na Fig. 6.4(a). Para os valores de $m_{L}$, verificamos no caso (i) para átomos de Fe na situação (3) o $m_{L}$ de $0,18 \mu_{B}$, enquanto para os outras situações um valor de $0,17 \mu_{B}$. Os valores de $m_{L}$ do Co têm os seguintes valores para o caso (i): (1) $0,26 \mu_{B}$, (2) $0,24 \mu_{B}$ e (3) $0,23 \mu_{B}$. Os valores de $m_{L}$ para os átomos de Fe no caso (ii) são independentes das vizinhanças e ficam em torno de 0,20 $\mu_{B}$. Entretanto, os átomos de Co extremos dependem de seus $N N$ : nas situações [(ii)-I] $0,35 \mu_{B}$ e [(ii)-II] $0,31 \mu_{B}$.

Usando um modelo proposto por H. Ebert et al., [135] podemos entender o comportamento do $m_{L}$ com a vizinhança. Neste modelo, o valor de $m_{L}$ é 
reforçado quando a $L D O S$ localizada na $\mathrm{E}_{F}$ é alta. Por um lado, verificamos, na Fig. 6.7, que a contribuição da banda $3 \mathrm{~d} \uparrow$ é muito pequena, em todos os casos, por estar totalmente preenchida. Por outro lado, a banda $3 \mathrm{~d} \downarrow$ é responsável para os diferentes valores de $m_{L}$, pois apresentam picos na $\mathrm{E}_{F}$. Os picos das LDOS nos átomos de Co internos são maiores que os dos $\mathrm{Fe}$ internos. Desta maneira, o valor de $m_{L}$ dos Co são maiores [Fig.6.7(a)-(b)]. Verificamos através das $L D O S$ que o pico na $\mathrm{E}_{F}$ dos átomos de Co com dois NN Co [Fig.6.7(b)] é o mais alto e decresce para outros casos, o que corresponde ao fato de que estes Co têm maiores valores de $m_{L}$. Os átomos que estão localizados nas extremidades das cadeias [Fig.6.7(c)-(d)] têm os picos das LDOS maiores quando comparados aos átomos internos e, correspondentemente apresentam maiores valores de $m_{L}$. Os valores individuais do
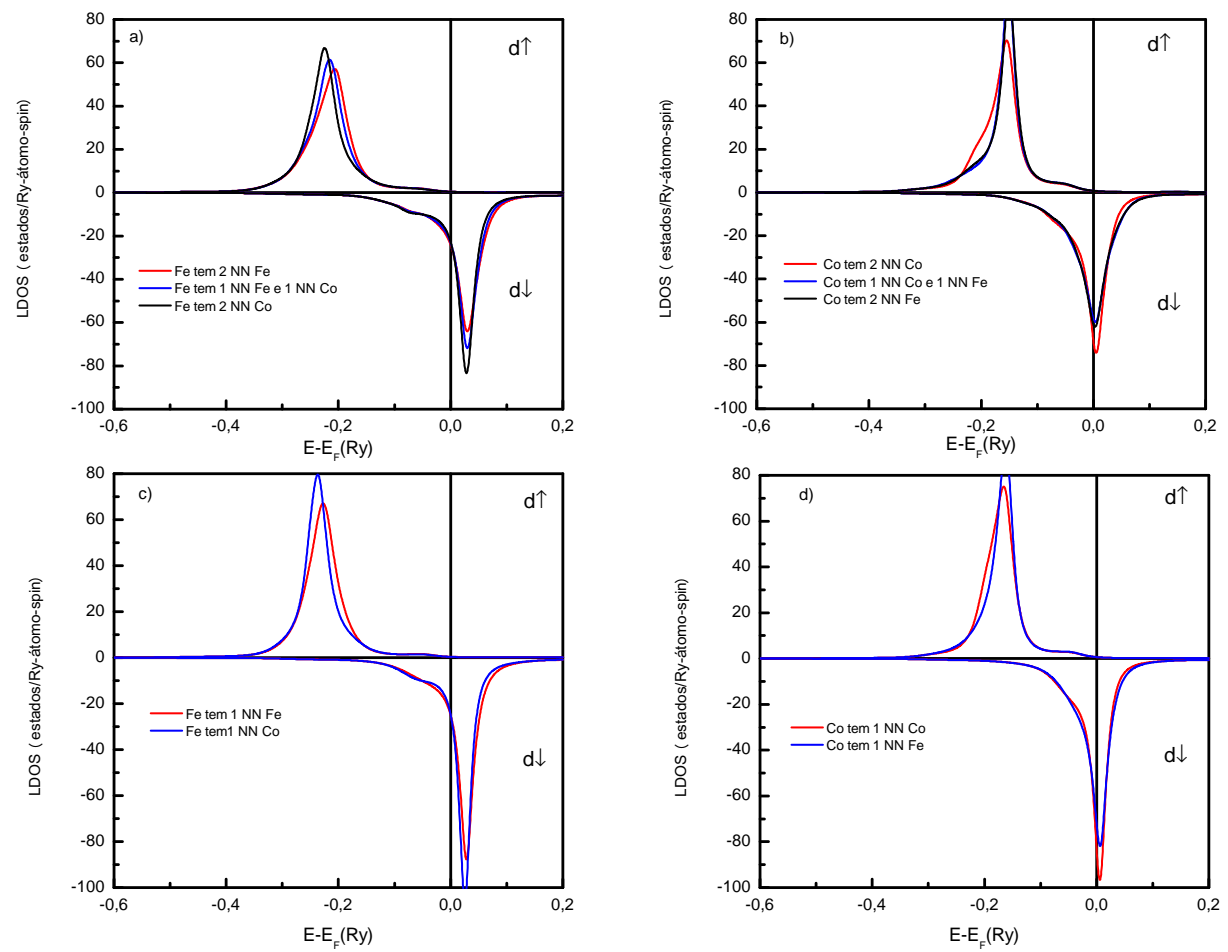

Figura 6.7: Densidades de estados locais projetadas nos sítios de Fe e Co localizados na posição interna da cadeia linear (a) e (b) na extremidade da cadeia linear (c) e (d) em função de diferentes tipos de vizinhos. 
momento magnético da Pt em função da vizinhança também foram investigados. Nossos resultados de $m_{s}$ e $m_{L}$ para átomos de Pt com um $N N$ Fe ou Co são $0,12 \mu_{B}$ e 0,03 $\mu_{B}$, respectivamente, enquanto que para átomos de $\mathrm{Pt}$ com dois $N N$ Fe ou Co são $0,18 \mu_{B}$ e $0,05 \mu_{B}$, respectivamente. 


\section{Capítulo 7}

\section{Conclusões}

Nesta tese, tivemos como objetivo investigar a estrutura eletrônica e propriedades magnéticas de materiais metálicos nanoestruturados depositados em superfície metálicas, utilizando o método computacional ab initio RSLMTO-ASA. Especificamente, estudamos as propriedades magnéticas de nanoestruturas de Mn depositados em Fe(001), nanofios de Mn em Fe(110) e nanofios de $\mathrm{Fe}_{x} \mathrm{Co}_{1-x}$ depositados em uma superfície $\mathrm{Pt}(111)$. Colocamos então nesta ordem as nossas conclusões:

Estudamos várias nanoestruturas magnéticas na forma de fios e aglomerados de Mn (variando de um até nove átomos de Mn) adsorvidos em Fe(001). Nossos cálculos nestas nanoestruturas de Mn indicam que as interações entre pares Mn e Fe dependem não somente da distância interatômica, mas fortemente com o meio local (tipo e quantidade de primeiros vizinhos). Uma possível explicação de como as configurações magnéticas evoluem a partir do adátomo Mn para filmes ultrafinos depositados em $\mathrm{Fe}(001)$ foi apresentada. As energias dos estados magnéticos colineares para estas nanoestruturas apresentam vários mínimos, fornecendo múltiplas configurações magnéticas não colineares que competem em energia. Contudo, foi obtido através dos nossos cálculos que as estabilidades dos estados fundamentais não colineares dependem do grau de interdifusão entre os átomos de Mn e Fe. A possibilidade do magnetismo não colinear revela uma nova estrutura magnética, incluindo uma estrutura de spin helicoidal para longos nanofios. Também, o aglome- 
rado em forma de cruz com cinco átomos de Mn apresenta uma intrigante configuração magnética não colinear, que pode ser descrita por uma estrutura topológica, como um half-skyrmion.

Nossas investigações de nanofios de $\mathrm{Mn}$ em Fe(110) indicam que as interações de troca magnética entre os momentos magnéticos de Mn-Mn primeiros vizinhos são fortemente negativas e decrescem rapidamente para pares Mn-Mn mais distantes. As interações de troca magnética Mn-Fe são também negativas (de magnitude muito menor que as $J_{M n-M n}$ primeiros vizinhos) e as interações de segundos vizinhos Mn-Fe são positivas. Nossos cálculos indicam que a configuração magnética do estado fundamental para os fios maiores que três átomos de Mn são não colineares. Este tipo de situação difere dos nanofios de Mn em Fe(001) que tem várias configurações magnéticas com energias muito próximas.

Nossas investigações para os nanofios de $\mathrm{Fe}_{x} \mathrm{Co}_{1-x}$ depositados em uma superfície Pt(111) mostram que os $m_{s}$ dos átomos de Fe e Co são independentes da concentração de Fe, por causa de que as bandas majoritárias são totalmente preenchidas. Em contraste com a liga FeCo $b c c$, o $m_{s}$ médio do nanofio varia linearmente com a concentração de Fe. O momento magnético de spin dos átomos de Fe e Co são consideravelmente aumentados quando comparados aos valores de bulk, aumentando $59 \%$ e 10\%, respectivamente. Esta mesma tendência (função linear com a concentração de $x$ ) foi também obtida para a monocamada de $\mathrm{Fe}_{x} \mathrm{Co}_{1-x}$ depositado em $\mathrm{Pt}(111)$ [53]. Nossos resultados para o $m_{L}$ médio são linearmente decrescente com $x$, diferentemente do comportamento calculado para a monocamada de $\mathrm{Fe}_{x} \mathrm{Co}_{1-x}$ sobre Pt(111), onde este sistema apresenta um máximo para o momento magnético orbital médio em $x=0,5$.

Por fim, em virtude do grande interesse no estudo de nanoestruturas metálicas depositadas em superfície metálicas, esperamos que nossos resultados motivem estudos experimentais tanto das nanoestruturas aqui estudadas como de outras nanoestruturas magnéticas. 


\section{Apêndice A}

\section{Orbitais Lineares Muffin-tin}

Neste apêndice, apresentaremos as diferentes bases nas quais o LMTOASA podem ser descritos, a saber: base canônica, ortogonal e Tight-Binding (TB). Um detalhamento mais geral deste formalismo pode ser encontrado nas Refs. [70,71].

\section{A.1 Introdução}

A teoria do funcional da densidade reduz o problema de muitos corpos para a solução de uma equação de um elétron $(|\psi\rangle)$ sob o potencial efetivo dos outros elétrons, onde pode ser expressa em um conjunto finito de base, $\left\{\chi_{i}\right\}$ da seguinte forma:

$$
|\psi\rangle=\sum_{i} u_{i}\left|\chi_{i}\right\rangle
$$

onde os coeficientes $\left\{u_{i}\right\}$ são os coeficientes da função de onda, e a partir disso podemos reescrever as equações de Kohn-Sham em uma forma matricial

$$
(H-\epsilon O) u=0
$$

$H$ é a matriz Hamiltoniana, $O$ é a matriz de overlap e $\epsilon$ são os autovalores. Os índices da matriz Hamiltoniana e overlap são definidos como:

$$
H_{i j}=\left\langle\chi_{i}|H| \chi_{j}\right\rangle
$$




$$
O_{i j}=\left\langle\chi_{i} \mid \chi_{j}\right\rangle
$$

Existe uma grande variedade de conjunto de base, contudo, para este trabalho uma base eficiente e localizada é necessária no cálculo da estrutura eletrônica no espaço direto. O formalismo LMTO foi primeiramente desenvolvido na chamada base canônica. Posteriormente foi mostrado por O. K. Andersen [65] que este formalismo pode ser descrito em termos de outras bases. Assim, tem-se a liberdade de escolher a base que seja mais apropriada para tratar cada caso. Existem duas bases de maior interesse, que são a base ortogonal, onde as funções de onda são ortogonais entre si, e a base mais localizada TB obtida de maneira a termos interações entre sítios vizinhos com o menor alcance possível.

Dentro do LMTO-ASA são usadas algumas aproximações que visam simplificar o problema de cálculo de estrutura eletrônica. A primeira delas é a aproximação da esfera atômica (ASA - Atomic Sphere Approximation) que considera o cristal totalmente preenchido por esferas centradas em cada átomo do material, onde as regiões entre as esferas (interstícios) e de sobreposição entre as esferas (overlaps) são desconsideradas. O raio de cada esfera, $s$, é dado por um valor apropriado obtido de modo que a soma dos volumes de todas as esferas seja igual ao volume ocupado pelos átomos no material. No caso de um material com apenas um tipo de átomo este será igual ao raio de Wigner-Seitz do sólido. Esta aproximação funciona muito bem para materiais densamente empacotados.

Consideramos também que cada esfera centrada nos sítios $R$ tem um potencial $V(r)$ esfericamente simétricos. Na ASA, onde não são considerados interstícios, o potencial será dado por:

$$
V=\sum_{R} V_{R}(r)
$$

Uma vez apresentadas as primeiras aproximações, sigamos com a determinação do conjunto de funções de base. 


\section{A.2 O conjunto de bases LMTO}

\section{A.2.1 Obtenção da Base Canônica}

A escolha do conjunto de funções de base $\left\{\chi_{i}\right\}$ deve ser feita de maneira conveniente para que tenhamos uma matriz Hamiltoniana (eq. A.3) e uma matriz de sobreposição (eq. A.4) com as mínimas dimensões possíveis, isto é, esperamos obter um conjunto de funções de base mínimo onde apenas um orbital por valor de $L=l, m$ seja suficiente para uma boa descrição do sistema. No caso dos metais de transição teremos nove orbitais por sítio (um $s$, três $p$ e cinco $d$ ).

O conjunto de base LMTO pode ser obtido através da construção de uma esfera de raio $s$ ao redor de um sítio atômico localizado em R. Como foi dito na seção anterior dentro da esfera é considerado um potencial muffin-tin $V_{M T}(\vec{r})$ que é esfericamente simétrico $V(r)$. Fora da esfera consideramos o zero do potencial muffin-tin (valor constante), $V_{M T Z}$ :

$$
V_{M T}(\vec{r})= \begin{cases}V(r) & r \leq s \\ V_{M T Z} & r \geq s\end{cases}
$$

onde $r=|\vec{r}-\vec{R}|$. Consideramos a equação tipo-Schrödinger dentro de uma única esfera muffin-tin centrada na posição $\vec{R}$ (esfera muffin-tin isolada)

$$
\left[-\nabla^{2}+V_{M T}(\vec{r})\right] \varphi_{\vec{R}}(\vec{r}, \varepsilon)=\varepsilon \varphi_{\vec{R}}(\vec{r}, \varepsilon)
$$

neste caso, podemos separar a solução em uma parte radial e angular:

$$
\varphi_{\vec{R} L}(\vec{r}, \varepsilon)=\varphi_{\vec{R} l}(r, \varepsilon) Y_{L}(\hat{r})
$$

onde $Y_{L}(\hat{r})$ é a função harmônica esférica com números quânticos $L=l, m$ e as funções $\varphi_{\vec{R} l}(r, \varepsilon)$ são as soluções radiais da equação tipo-Schrödinger que são obtidas através de métodos numéricos.

Na região fora da esfera muffin-tin o potencial é constante, as soluções são determinadas pela equação de Helmholtz. Podemos considerar que nesta região $V_{M T}-\varepsilon=0$, desta forma a equação tem duas soluções linearmente 
independentes, que são as funções esféricas de Bessel $J_{\vec{R}, l}(r)$ e Neumann $K_{\vec{R}, l}$ dadas por:

$$
\left\{\begin{array}{l}
J_{\vec{R} l}=\frac{1}{2(2 l+1)}\left(\frac{r}{s}\right)^{l} \\
K_{\vec{R} l}=\left(\frac{s}{r}\right)^{l+1} .
\end{array}\right.
$$

Usando a condição que $r \rightarrow \infty$ a solução radial tem que se anular. Assim, escrevemos a solução fora da esfera como:

$$
K_{\vec{R} l}=\left(\frac{s}{r}\right)^{l+1} Y_{L}(\hat{r}) \quad r>s .
$$

\section{A.2.2 Aproximação da esfera atômica}

Um sistema periódico cristalino densamente empacotado (close packed) pode ser preenchido por várias esferas muffin-tin, e desconsideramos, como dito anteriormente, as regiões de interstícios. Nosso objetivo é construir funções de base para descrever este sistema. Para isso tomamos a função $K_{\vec{R} L}$, relacionado ao centro do sítio $R$, conforme descrita anteriormente para o potencial muffin-tin isolado, e substituir a parte de $K_{\vec{R} L}$ que penetra no interior das esferas centradas nos sítios $R^{\prime} \neq R$ por funções relacionadas com a solução da equação tipo-Schrödinger radial dentro da esfera $R^{\prime}$ de modo que as derivadas logarítmicas nos contornos das esferas sejam contínuas. Dentro deste contexto, definimos as chamadas funções envelope que têm como objetivo estabelecer as condições de contorno nas esferas quando tomarmos as soluções da equação de tipo-Schrödinger para descrever o problema no interior das mesmas. No caso da base canônica o conjunto de funções envelope é definido pela função $K_{\vec{R} L}\left(\vec{r}_{R}\right)$, dada anteriormente, e que pode ser reescrita como:

$$
K_{\vec{R} L}\left(\vec{r}_{R}\right)=k_{\vec{R} L}\left(\vec{r}_{R}\right) Y_{L}\left(\hat{r}_{R}\right)
$$

onde a parte radial é dada por:

$$
k_{\vec{R} L}\left(\vec{r}_{R}\right)=\left(\frac{s}{r}\right)^{l+1} .
$$

A eq. A.9 serve para estabelecer o comportamento das funções de base canônica $\chi_{R L}^{0}$, o índice 0 denota a base canônica. O fato de esta função divergir na origem não será relevante, pois no processo de obtenção das funções 
de base canônica $\chi_{R L}^{0}$ substituímos a parte da função envelope (eqs.A.9) que entra nas esferas $R^{\prime}$ por funções relacionadas a solução da equação tipoSchrödinger dentro destas esferas para um potencial esfericamente simétrico a ser obtido por autoconsistência. Estas funções são ortogonais aos níveis de caroço na região do sítio $R^{\prime}$, constituindo uma boa aproximação para a solução do problema nessa região.

Neste processo, também devemos levar em consideração as condições de continuidade e diferenciabilidade no contorno das esferas. Este procedimento é chamado de augment, sendo feito com o auxílio da função envelope. Neste procedimento expandimos a função envelope em torno dos sítios $R^{\prime}$, uma vez que a solução da equação tipo-Schrödinger é definida em torno de $R^{\prime}$. Expandimos a parte de $K_{\vec{R} L}\left(\vec{r}_{R}\right)$ que se estende nas esferas centradas em $R^{\prime} \neq R$, em termos das soluções da equação de Laplace regulares na origem e centradas em cada sítio $R^{\prime} L^{\prime}$, como segue:

$$
K_{R L}^{0}=-\sum_{R^{\prime} L^{\prime}} J_{R^{\prime} L^{\prime}}^{0}\left(r_{R^{\prime}}\right) S_{R^{\prime} L^{\prime}, R L}^{0} \quad r>s,
$$

onde as funções $J_{R^{\prime} L^{\prime}}^{0}\left(r_{R^{\prime}}\right)$ são nulas fora das esferas $R^{\prime}$ e no interior das mesmas são dadas por:

$$
J_{R^{\prime} L^{\prime}}^{0}\left(r_{R^{\prime}}\right)=\frac{1}{2\left(2 l^{\prime}+1\right)}\left(\frac{r_{R^{\prime}}}{s}\right)^{l^{\prime}} .
$$

Na eq. A.11 os coeficientes da expansão, $S_{R^{\prime} L^{\prime}, R L}^{0}$, dependem apenas das distâncias entre os sítios $R$ e $R^{\prime}$, anulando-se em $R^{\prime}=R$. $S^{0}$ é denominada matriz de estrutura canônica, e seus elementos são coeficientes da expansão da função $K_{R L}$ em torno de $R^{\prime}$ dados por:

$$
S_{R^{\prime} L^{\prime}, R L}^{0}=\frac{1}{(4 \pi)^{1 / 2}} G_{l^{\prime} m^{\prime}, l m}\left|\frac{\vec{R}-\vec{R}^{\prime}}{s}\right|^{-l^{\prime}-l-1} Y_{l+1, m^{\prime}-m}^{*}{ }^{\left(R-R^{\prime}\right)},
$$

onde

$G_{l^{\prime} m^{\prime}, l m}=(-1)^{l+m+1}\left[\frac{\left(2 l^{\prime}+1\right)(2 l+1)\left(l+l^{\prime}+m^{\prime}-m\right) !\left(l+l^{\prime}-m^{\prime}+m\right) !}{\left(2 l^{\prime}+2 l+1\right)\left(l^{\prime}-m^{\prime}\right) !\left(l^{\prime}-m^{\prime}\right) !(l+m) !(l-m) !}\right]^{1 / 2}$. 
Podemos escrever a função envelope associada em todo o espaço, denotando por $K_{R L}^{\infty}$, em termos de $K_{R L}$ centrada em $R$ (eq. A.9) e na expansão das demais esferas (eq. A.11)

$$
K_{R L}^{\infty}=K_{R L}-\sum_{R^{\prime} L^{\prime}} J_{R^{\prime} L^{\prime}}^{0}\left(r_{R^{\prime}}\right) S_{R^{\prime} L^{\prime}, R L}^{0}
$$

nesta equação consideramos que a função harmônica esférica esta implicitamente incluído. Nas equações acima definimos:

$$
k_{R l}=\left(\frac{r_{R^{\prime}}}{s}\right)^{-l-1}
$$

e

$$
j_{R^{\prime} L^{\prime}}^{0}\left(r_{R^{\prime}}\right)=\frac{1}{2\left(2 l^{\prime}+1\right)}\left(\frac{r_{R^{\prime}}}{s}\right)^{l^{\prime}+1},
$$

onde estas funções são definidas como nulas fora das esferas que estão centradas.

Na notação de Dirac podemos escrever a função envelope como:

$$
|K\rangle^{\infty}=|K\rangle-\left|J^{0}\right\rangle S^{0}
$$

onde $|K\rangle,\left|J^{0}\right\rangle$ e $|K\rangle^{\infty}$ são vetores linhas com componentes $\left|K_{R L}\right\rangle,\left|J_{R^{\prime} L^{\prime}}^{0}\right\rangle$ e $\left|K_{R L}\right\rangle^{\infty}$, respectivamente. Similarmente, $\langle K|,\left\langle J^{0}\right| \mathrm{e}^{\infty}\langle K|$ são vetores coluna, enquanto $S^{0},{ }^{\infty}\langle K \mid K\rangle^{\infty}$ e etc, são matrizes.

Obtida a função envelope $K_{R L}^{\infty}$ prosseguimos a realização do augment que consiste em substituir a parte da função envelope no interior das esferas $R^{\prime}$ por funções relacionadas às soluções da equação tipo-Schrödinger dentro das mesmas, com o potencial esfericamente simétrico, conservando as condições impostas pela função envelope. Definimos, desta forma, as soluções da equação tipo-Schrödinger radial dentro de cada esfera $R$ como $\varphi_{R l}(r, E)$. Calculamos as funções e suas derivadas em relação à energia para uma energia $E=E_{\nu}$ :

$$
\begin{gathered}
\varphi_{R l}(r, E)=\varphi_{R l}\left(r, E_{\nu}\right), \\
\dot{\varphi}_{R l}(r, E)=\left.\frac{\partial}{\partial E} \varphi_{R l}(r, E)\right|_{E=E_{\nu}},
\end{gathered}
$$

onde $E=E_{\nu}$ é a energia do centro da banda ocupada. 
Deste modo, podemos escrever a função de base em todo o espaço, $\chi_{R L}^{0}{ }^{\infty}$, em termos de uma combinação linear de $\varphi_{R l}(r)$ e $\dot{\varphi}_{R l}(r)$ que são independentes da energia:

$$
\chi_{R L}^{0}{ }^{\infty}=\varphi_{R l} Y_{L}\left(\hat{r}_{R}\right)+\sum_{R^{\prime} l^{\prime}} \dot{\varphi}_{R^{\prime} l^{\prime}}\left(r_{R}\right) Y_{L^{\prime}}\left(\hat{r}_{R^{\prime}}\right) h_{R^{\prime} L^{\prime}, R L}^{0}
$$

onde

$$
\dot{\varphi}_{R^{\prime} l^{\prime}}\left(r_{R}\right)=\dot{\varphi}_{R^{\prime} l^{\prime}}^{0}\left(r_{R}\right)+\varphi_{R^{\prime} l^{\prime}} O_{R^{\prime} l^{\prime}}^{0}
$$

$\mathrm{ou}$

$$
\chi_{R L}^{0}{ }^{\infty}=\varphi_{R l} Y_{L}\left(\hat{r}_{R}\right)+\sum_{R^{\prime} l^{\prime}}\left[\dot{\varphi}_{R^{\prime} l^{\prime}}^{0}\left(r_{R}\right)+\varphi_{R^{\prime} l^{\prime}} O_{R^{\prime} L^{\prime}}^{0}\right] Y_{L^{\prime}}\left(\hat{r}_{R^{\prime}}\right) h_{R^{\prime} L^{\prime}, R L}^{0}
$$

em notação vetorial:

$$
\left|\chi^{0}\right\rangle^{\infty}=|\chi\rangle\left(1+o^{0} h^{0}\right)+|\dot{\chi}\rangle h^{0}
$$

nesta equação $h^{0}$ e $o^{0}$ são matrizes que dependem das condições de contorno nas esferas. Na equação acima as partes angulares já estão incluídas.

As funções $\dot{\varphi}(r)$ e $\varphi(r)$ são definidas como zero fora da esfera a que se referem. Essas funções tem as seguintes propriedades [70]: assumindo que $\varphi_{R l}(E, r)$ seja normalizada à unidade na esfera $\mathrm{R}$ obtem-se que $\varphi_{R l}(E, r)$ é ortogonal a $\dot{\varphi_{R l}}(E, r)$ (relação também válidas para $E=E_{\nu}$ ). Outra propriedade importante é que as funções $\chi_{R L}^{0}{ }^{\infty}$ são ortogonais aos estados de caroço dentro de todas as esfera $R^{\prime}$. Desse modo, as autofunções $\psi_{j}$ (eq. A.1) podem ser descritas em termos de um conjunto com um número não muito grande de funções de base.

Por fim, os coeficientes da eq. A.24 que definem a base canônica $\chi_{R L}^{0}{ }^{\infty}$ são determinados a partir da condições de contorno impostas pela função envelope $K_{R L}^{\infty}$.

Para determinação das matrizes $h^{0}$ e $o^{0}$ devemos conectar as funções radiais da função envelope $k_{R l}$ (eq. A.16) e $j_{R l}^{0}$ (eq. A.17) com a combinação linear de $\varphi_{R l}(r)$ e $\dot{\varphi}_{R l}$ de tal forma que essas funções caiam uma sobre outra em $r=s$. Para fazer isto, utilizamos o wronskianos para o casamento de 
uma função $f(r)$ como uma combinação linear de duas funções $a(r)$ e $b(r)$ em um dado ponto, como por exemplo em $r=s$, definido como:

$$
W(a, b)=s^{2}\left[a(s) b^{\prime}(s)-a^{\prime}(s) b(s)\right]
$$

Assim temos:

$$
h^{0}=\left[-\frac{W(k, \varphi)}{W\left(k, \varphi^{0}\right)}+\left(\frac{2}{a}\right)^{1 / 2} W\left(j^{0}, \varphi\right) S^{0} W\left(j^{0}, \varphi\right)\left(\frac{2}{a}\right)^{1 / 2}\right]
$$

e

$$
o^{0}=-\frac{W\left(j^{0}, \dot{\varphi}\right)}{W\left(j^{0}, \varphi\right)}
$$

Nestas expressões omitimos os índices $R L$ para não carregar a notação. De posse dos coeficientes $o^{0}$ e $h^{0}$, a base canônica $\left|\chi^{0}\right\rangle^{\infty}$ está completamente determinada. Contudo, no formalismo LMTO-ASA é comum descrever o coeficiente $h^{0}$ em termos que dependem e independem do potencial. Para isso introduzimos os parâmetros $C^{0}$ e $\Delta^{0}$, chamados de parâmetros de potencial, na equação para $h^{0}$ (eq. A.26) para reescrevê-la da seguinte forma:

$$
h_{R L, R^{\prime} L^{\prime}}^{0}=\left(C_{R l}^{0}-E_{\nu R l}\right) \delta_{R, R^{\prime}} \delta_{L, L^{\prime}}+\Delta_{R l}^{0}{ }^{1 / 2} S_{R L, R^{\prime} L^{\prime}}^{0} \Delta_{R l}^{0}{ }^{1 / 2},
$$

onde são definidos:

$$
C_{R l}^{0}=E_{\nu R l}-\frac{W(k, \varphi)}{W\left(k, \varphi^{0}\right)}
$$

e

$$
\Delta_{R l}^{01 / 2}=\left(\frac{2}{a}\right)^{1 / 2} W\left(j^{0}, \varphi\right) .
$$

Portanto, $h^{0}$ é expresso agora em termos da matriz $S^{0}$ (eq. A.13) que dependem somente das posições atômicas, $R$ e $R^{\prime}$ e independem dos potenciais nas esferas atômicas e de seus raios; e dos parâmetros $C^{0}$ e $\Delta^{0}$ que dependem das condições de contorno nas esferas através dos wronskianos e, portanto, dependem do potencial.

A partir das funções de base podemos obter a matriz Hamiltoniana e a de overlap na base canônica:

$$
H^{0}={ }^{\infty}\left\langle\chi^{0}\left|-\nabla^{2}+V\right| \chi^{0}\right\rangle^{\infty}
$$


e

$$
O^{0}={ }^{\infty}\left\langle\chi^{0} \mid \chi^{0}\right\rangle^{\infty}
$$

Substituindo a eq. A.24 nas expressões acima e usando as propriedades das funções de $|\varphi\rangle$ e $|\dot{\varphi}\rangle[65]$ podemos obter as seguintes funções:

$$
\begin{aligned}
& H^{0}=h^{0}+h^{0+} o^{0+} h^{0}+E_{\nu} O^{0}, \\
& O^{0}=1+o^{0} h^{0}+\left(o^{0} h^{0}\right)^{+}+\left(o^{0} h^{0}\right)^{+} o^{0} h^{0} .
\end{aligned}
$$

Nessas equações termos muito pequenos, da ordem de $\left(h^{0}+p h^{0}\right) \operatorname{com} p_{R L}=$ $\left|\dot{\varphi}^{2}\right\rangle$, foram desprezados. Desta forma, obtidos a matiz Hamiltoniana e de overlap na base canônica podemos escrever a equação secular do LMTO:

$$
\left(H^{0}-E_{j} O^{0}\right) u_{j}^{0}=0
$$

a partir da qual encontramos os autovalores na base canônica. Contudo, o procedimento de espaço direto só será viável se a matriz Hamiltoniana for localizada e esta característica não está garantida ao utilizarmos a base canônica. Isto porque a matriz canônica $S^{0}$ que está relacionada com a Hamiltoniana (ver eqs.A.26 e A.33), tem um decaimento muito lento com a distância (proporcional a $r^{-2 l-1}$ ). Para contornar essa questão, podemos obter no formalismo LMTO-ASA funções mais apropriadas ao problema. Nas próximas seções apresentamos como fazer isso.

\section{A.2.3 Formalismo LMTO-ASA numa Base Genérica}

Uma das vantagens do LMTO-ASA, como foi descrito no início do capítulo, é o fato de poder ser escrito em termos de outras bases. Assim, para obter uma nova base, usualmente se constrói primeiramente um novo conjunto de funções envelope em uma base genérica. Este novo conjunto pode ser dado por uma justaposição de funções envelope da base canônica. Em seguida, procede-se analogamente à construção da base canônica, realizando o augment, onde nas regiões interiores as esferas centradas no sítio $R^{\prime}$ o conjunto de funções envelope é substituído por combinações de funções regulares $\varphi(r)$ e $\dot{\varphi}(r)$. 
Para escrevermos a base LMTO-ASA na base genérica (denotada pelo superescrito G) definimos o chamado parâmetro de mistura, $Q^{G}$. Nesta representação a função envelope $K_{R L}^{G}{ }^{\infty}$ é dada, em analogia ao caso canônico, por:

$$
K_{R L}^{G}{ }^{\infty}\left(\hat{r}_{R}\right)=K_{R L}\left(\hat{r}_{R}\right)-\sum_{R^{\prime} L^{\prime}} J_{R^{\prime} L^{\prime}}^{G}\left(\hat{r}_{R^{\prime}}\right) S_{R^{\prime} L^{\prime}, R L}^{G} .
$$

A nova base é obtida misturando uma fração $Q_{R^{\prime} l^{\prime}}^{G}$ de soluções irregulares $K_{R^{\prime} L^{\prime}}\left(\hat{r}_{R}\right)$ às soluções nas esferas $R^{\prime}$, ou seja:

$$
J_{R^{\prime} L^{\prime}}^{G}\left(\hat{r}_{R^{\prime}}\right)=J_{R^{\prime} L^{\prime}}^{0}\left(\hat{r}_{R^{\prime}}\right)-K_{R^{\prime} L^{\prime}}\left(\hat{r}_{R^{\prime}}\right) Q_{R^{\prime} L^{\prime}}^{G}
$$

Essas equações podem ser escritas em uma forma vetorial da seguinte forma:

$$
\begin{aligned}
& \left|K^{G}\right\rangle=|K\rangle-\left|J^{G}\right\rangle S^{G} \\
& \left|J^{G}\right\rangle=\left|J^{0}\right\rangle-|K\rangle Q^{G}
\end{aligned}
$$

onde os índices são omitidos para não carregar a notação. O parâmetro $Q^{G}$ define a base em questão, por exemplo, a base canônica é obtida tomando-se $Q^{G}=0$.

Comparando a eq. A.18 com as eqs.A.38 e A.39 obtemos a matriz de estrutura genérica $S^{G}$ em termos da matriz de estrutura canônica:

$$
S^{G}=S^{0}\left(1-Q^{G} S^{0}\right)^{-1}
$$

onde 1 é a matriz unitária. E a relação entre as funções é dada por:

$$
\left|K^{G}\right\rangle^{\infty}=|K\rangle^{\infty}\left(1-Q^{G} S^{0}\right)^{-1}
$$

Na equação acima $S^{0}$ depende apenas da distância entre os sítios, deste modo, uma particular base é definida pelo parâmetro de mistura $Q^{G}$.

De um modo similar ao que foi feito para a base canônica, podemos definir a função de base $\left|\chi^{G}\right\rangle^{\infty}$, em função dos parâmetros $h^{G}$ e $o^{G}$ :

$$
\left|\chi^{G}\right\rangle^{\infty}=|\varphi\rangle+\left|\dot{\varphi}^{G}\right\rangle h^{G}
$$

e

$$
\left|\dot{\varphi}^{G}\right\rangle=|\dot{\varphi}\rangle+|\varphi\rangle_{O}^{G}
$$


que pode ser escrito como:

$$
\left|\chi^{G}\right\rangle^{\infty}=|\varphi\rangle\left(1+o^{G} h^{G}\right)+|\dot{\varphi}\rangle h^{G}
$$

Os parâmetros $h^{G}$ e $o^{G}$ são obtidos de forma análoga ao caso da base canônica:

$$
h^{G}=\left[-\frac{W(k, \varphi)}{W\left(k, \varphi^{G}\right)}+\left(\frac{2}{a}\right)^{1 / 2} W\left(j^{G}, \varphi\right) S^{G} W\left(j^{G}, \varphi\right)\left(\frac{2}{a}\right)^{1 / 2}\right]
$$

$\mathrm{e}$

$$
o^{G}=-\frac{W\left(j^{G}, \dot{\varphi}\right)}{W\left(j^{G}, \varphi\right)}=-\frac{W\left(j^{0}, \dot{\varphi}\right)-W(k, \dot{\varphi}) Q^{G}}{W\left(j^{0}, \varphi\right)-W(k, \varphi) Q^{G}}
$$

onde $o^{G}$ é uma matriz diagonal com elementos $o_{R l}^{G}$ e $h^{G}$ é uma matriz com elementos $h_{R L, R^{\prime} L^{\prime}}^{G}$. Nesta última equação podemos verificar que os parâmetros $o^{G}$ e $Q^{G}$ estão diretamente relacionados, ou seja, definindo-se um o outro está automaticamente definido.

Por fim, com a função de base $\left|\chi^{G \infty}\right\rangle$ em termos de $h^{G}$ e $o^{G}$ determinamos as matrizes Hamiltoniana $\left(H^{G}\right)$ e de overlap $\left(O^{G}\right)$ na base genérica, a saber:

$$
\begin{gathered}
H^{G}=h^{G}+h^{G^{+}} o^{G+} h^{G}+E_{\nu} O^{G}, \\
O^{G}=1+o^{G} h^{G}+\left(o^{G} h^{G}\right)^{+}+\left(o^{G} h^{G}\right)^{+} o^{G} h^{G} .
\end{gathered}
$$

O procedimento desenvolvido nesta seção nos dá expressões de $\left|\chi^{G}\right\rangle^{\infty}, H^{G}$ e $O^{G}$ para o LMTO-ASA válidas para qualquer valor do parâmetro $Q^{G}$ (ou de $\left.o^{G}\right)$. Prosseguiremos definindo outras bases de interesse escolhendo valores convenientes de $Q^{G}$ (ou de $o^{G}$ ).

\section{A.2.4 Base Localizada - TB}

Podemos determinar um valor de $Q^{G}$ de forma que a base em questão seja a mais localizada possível, isto é, de forma que as interações entre os vizinhos mais próximos sejam suficientes para uma boa descrição do sistema. Desta forma, com uma matriz Hamiltoniana descrita em termos de bases mais localizadas possibilita o uso do Método de Recorrência (apêndice B) que é mais conveniente para se tratar problemas no espaço direto. Observando as 
eqs. A.40 e A.41 que definem a base genérica, verificamos que a base será localizada se a matriz de estrutura $S^{G}$ liga apenas sítios próximos.

Na literatura [65] existem valores tabelados para $Q^{G}$, que independem da estrutura do material, de forma que a matriz de estrutura seja localizada. Uma importante propriedade da matriz de estrutura localizada é que esses valores decrescem exponencialmente com a distância entre os sítios. Passemos a denotar esses parâmetros acom o superscrito barra, $\bar{Q}$, tendo os seguintes valores:

$$
\begin{gathered}
\bar{Q}_{s}=0,3845 \\
\bar{Q}_{p}=0,05303 \\
\bar{Q}_{d}=0,010714 .
\end{gathered}
$$

No caso de metais, em geral, tem-se interesse somente nos orbitais $s, p$ e $d$ e então consideramos $\bar{Q}_{l}=0$ para $l>2$. Através da eq. A.40 verificamos que, ao tomarmos valores constantes para $\bar{Q}$, a matriz $\bar{S}$ passa a depender somente da estrutura do material através de $S^{0}$. Na base mais localizada, chamada de base TB, a equação de autovalores é dada através dos parâmetros $\bar{h}$ e $\bar{o}$, determinados através das eqs.A.45 e A.46 para valores de $\bar{Q}$ definidos acima. Usando as eqs.A.47 e A.48 e trocando $h^{G}$ e $o^{G}$ respectivamente por $\bar{h}$ e $\bar{o}$, obtemos:

$$
\begin{gathered}
(\bar{H}-E \bar{O}) \bar{u}=\left[\bar{h}+\bar{h}^{+} \bar{o}^{+} \bar{h}+E_{\nu} \bar{O}-E \bar{O}\right] \bar{u}=0 \\
{\left[\bar{h}+\bar{h}^{+} \bar{o}^{+} \bar{h}+\left(E_{\nu}-E\right)\left(1+\bar{o} \bar{h}+(\bar{o} \bar{h})^{+}+(\bar{o} \bar{h})^{+} \bar{o} \bar{h}\right)\right]=0}
\end{gathered}
$$

\section{A.2.5 Base Ortogonal}

Nesta seção apresentamos a base Ortogonal, com o objetivo de simplificar o problema de autovalores. Para isso usando na eq. A.48 $o^{G}=0$ obtemos para a matriz de overlap $O^{G}=1$. Denotaremos os parâmetros na base Ortogonal sem índice superior. A matriz Hamiltoniana (eq. A.47) vai ser escrita na representação Ortogonal como:

$$
H=h+E_{\nu}
$$


e portanto a equação de autovalores será escrita na forma:

$$
H u=E u
$$

$\mathrm{ou}$

$$
\left(h+E_{\nu}\right) u=E u,
$$

isolando $h$ na equação acima obtemos:

$$
h=E-E_{\nu} .
$$

Escrevendo $H$ em termos dos chamados parâmetros de potencial na base Ortogonal $(C, \Delta$ e $Q)$ obtemos a expressão:

$$
H=C+\Delta^{1 / 2} S \Delta^{1 / 2},
$$

onde

$$
\begin{aligned}
C & =E_{\nu}-\frac{W(k, \varphi)}{W(k, \dot{\varphi})} \\
\Delta^{1 / 2} & =\left(\frac{2}{a}\right)^{1 / 2} W(j, \varphi) .
\end{aligned}
$$

A matriz de estrutura $S$ vai ser dada pela seguinte equação:

$$
S=S^{0}\left(1-Q S^{0}\right)^{-1}
$$

com o parâmetro $Q$ obtido pela escolha de $o^{G}=0$ na eq. A.46, assumindo o valor:

$$
Q=\frac{W(j, \dot{\varphi})}{W(k, \dot{\varphi})} .
$$

Nas equações acima se verifica que $S$ tem uma dependência da estrutura do material, pois o parâmetro $Q$ depende das condições de contorno na esfera muffin-tin. Portanto, a Hamiltoniana A.58 não pode ser dividida em uma parte dependente e outra independente do potencial.

Para determinarmos a função de base $|\chi\rangle^{\infty}$ (eq. A.44) tomamos $o^{G}=0$ e trocamos $h^{G}$ pela relação obtida em A.54, obtendo:

$$
|\chi\rangle^{\infty}=|\varphi\rangle+|\dot{\varphi}\rangle\left(H-E_{\nu}\right)
$$


que pode ser reescrita em termos dos autovalores de $\mathrm{H}$ :

$$
|\chi\rangle^{\infty}=|\varphi\rangle+|\dot{\varphi}\rangle\left(E-E_{\nu}\right)
$$

Na equação acima é interessante notar que no LMTO-ASA as funções de base na representação Ortogonal são dadas por uma expansão em série de Taylor até primeira ordem em energia de ondas parciais $|\varphi(E, r)\rangle$ em torno de uma dada energia $E_{\nu}$.

\section{A.2.6 Representação Ortogonal da Matriz Hamiltoni- ana Expressa em Termos de Parâmetros da Re- presentação Tight-Binding}

Nas seções anteriores desenvolvemos a função envelope genérica definida no conjunto de equações A.38 e A.39, e através do parâmetro de mistura poderíamos obter o formalismo LMTO-ASA na representação Ortogonal e TB. Essas transformações são necessárias para tratarmos problemas no espaço direto, pois com o uso da base Ortogonal simplificamos o problema de autovalores e a base TB possibilita o uso do Método de Recorrência (apêndice B). Nesta seção, apresentaremos as aproximações que nos permitem obter uma Hamiltoniana Ortogonal em termos dos parâmetros de potencial na representação TB.

Inicialmente expressamos a Hamiltoniana Ortogonal em termos da base geral. Isto pode ser feito comparando os termos em $|\dot{\varphi}\rangle$ nas bases Ortogonal (eq. A.63) e Geral (eq. A.44) obtendo:

$$
h=h^{G}\left(1+o^{G} h^{G}\right)^{-1} .
$$

A relação acima é válida para qualquer base. Portanto, a Hamiltoniana na base Ortogonal em termos da base TB pode ser escrita como:

$$
H=h+E_{\nu}=\bar{h}(1+\bar{o} \bar{h})^{-1}+E_{\nu}
$$

no caso de $(\bar{o} \bar{h})$ ser pequeno, podemos expandir $(1+\bar{o} \bar{h})^{-1}$ em série de potências de $\bar{o} \bar{h}$ e reescrever a Hamiltoniana como:

$$
H=E_{\nu}+\bar{h}(1-\bar{o} \bar{h}+\bar{o} \bar{h} \bar{o} \bar{h}+\ldots)
$$


$\mathrm{Ou}$

$$
H=E_{\nu}+\bar{h}+\bar{h}(1-\bar{o} \bar{h}+\bar{o} \bar{h} \bar{o} \bar{h}+\ldots) .
$$

Usando a relação que $\bar{h}=E-E_{\nu}$ e considerando que na maioria dos casos podemos tomar termos até primeira ordem em $\left(E-E_{\nu}\right)$, podemos reescrever:

$$
H=E_{\nu}+\bar{h}
$$

neste caso a Hamiltoniana dentro desta aproximação fornecem resultados corretos em energia até a primeira ordem de $\left(E-E_{\nu}\right)$. Na eq. A.68 a inclusão de mais termos apenas irá aumentar a precisão à medida que nos afastarmos da energia $E_{\nu}$, ou seja, usando-se $H$ na forma dada pela eq. A.69 se restringem àquelas em torno de $E_{\nu}$.

A eq. A.69 pode ser reescrita em termos dos parâmetros de potencial na base TB:

$$
H=\bar{C}+\bar{\Delta}^{1 / 2} \bar{S} \bar{\Delta}^{1 / 2},
$$

onde estes parâmetros de potencial são definidos como:

$$
\begin{aligned}
\bar{C} & =E_{\nu}-\frac{W(k, \varphi)}{W(k, \bar{\varphi})} \\
\Delta^{1 / 2} & =\left(\frac{2}{a}\right)^{1 / 2} W(\bar{j}, \varphi) .
\end{aligned}
$$

Os parâmetros de potencial na base ortogonal $(C, \Delta, Q)$ estão diretamente relacionados com a solução da equação tipo-Schrödinger em cada esfera $R$ e são calculados nos códigos LMTO-ASA de espaço $k$ usuais. Estes parâmetros se encontram tabelados na literatura para metais de transição e são o ponto de partida para cálculos parametrizados [65]. No Método de Recorrência são usados os parâmetros de potencial na representação Ortogonal. Assim, no RSLMTO-ASA é necessário usar uma expressão que relacione os parâmetros $C$, $\Delta$ e $Q$ da representação Ortogonal com os parâmetros na base TB de acordo com a seguinte relação [65]:

$$
\frac{\bar{\Delta}^{1 / 2}}{\Delta^{1 / 2}}=\left[1-(Q-\bar{Q}) \frac{C-E_{\nu}}{\Delta}\right]=\frac{\bar{C}-E_{\nu}}{C-E_{\nu}} .
$$


O cálculo da Hamiltoniana $\mathrm{H}$ de primeira ordem na base Ortogonal expressa em termos de parâmetros de potencial da base TB pode ser separado em duas partes. Uma parte depende do potencial em cada esfera e consiste na obtenção dos parâmetros $\bar{C}$ e $\bar{\Delta}$ (onde estes representam respectivamente o centro e a largura da banda relativa ao sítio R) que serão usados na Hamiltoniana H. A outra parte depende da estrutura do material, e consiste no cálculo da matriz de estrutura $\bar{S}$ que, como vimos, está relacionada com a distância entre os sítios e estabelece a conexão entre os mesmos. 


\section{Apêndice B}

\section{Método de Recorrência}

Mostramos que no formalismo LMTO-ASA podemos escrever a matriz Hamiltoniana em uma base ortogonal em termos de parâmetros TB. Nesta representação a Hamiltoniana é uma matriz $9 N X 9 N$, onde $\mathrm{N}$ o número de átomos do aglomerado e 9 o número de orbitais (um $s$, três $p$ e cinco $d$ ). Devido às dimensões desta matriz, é inviável resolver o problema de autovalores e calcular as densidades de estados como nos métodos tradicionais [65]. Assim, utiliza-se o Método de Recorrência, introduzido por R. Haydock et. al., [67] que possibilita esses cálculos para uma Hamiltoniana esparsa e pode ser utilizado para sistema sem periodicidade.

O Método de Recorrência consiste em efetuar uma mudança de base que permite reescrever a matriz Hamiltoniana em uma forma tridiagonal (matriz de Jacobi). Para isso consideramos um modelo, como colocado na Fig. B.1, onde um elétron em um estado inicial $u_{0}$ com um orbital por átomo. Representamos $u_{1}$ e $u_{2}$ estados que representam uma combinação linear de orbitais de átomos que são primeiros e segundos vizinhos do átomo que está localizado em $u_{0}$. O elétron em $u_{0}$ pode saltar para $u_{1}$, enquanto o elétron que esta em $u_{1}$, ele pode saltar para $u_{0}$ ou para $u_{2}$. Este modelo é chamado de modelo da cadeia (chain model) como mostrado na Fig. B.2.

Considerando que $u_{0}, u_{1}, u_{2}, \ldots, u_{N}$ são estados ortogonais, de tal forma que o elétron posicionado em um estado $u_{0}$ pode saltar para o seu vizinho $u_{1}$, onde elétrons que estão no meio da cadeia, $u_{n}$, podem saltar para os vizinhos 


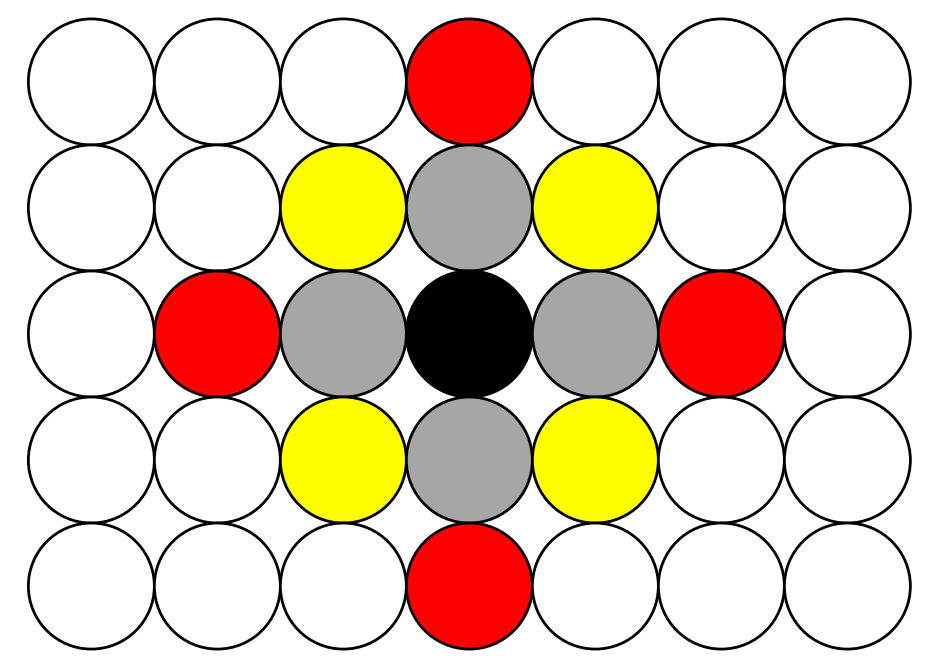

Figura B.1: Figura esquemática dos estados expandidos no espaço nas vizinhanças do átomo central. Aqui o estado inicial $u_{0}$ é o circulo preto, seus vizinhos são representados por $u_{1}$ (esfera cinza) e $u_{2}$ (esfera amarela).

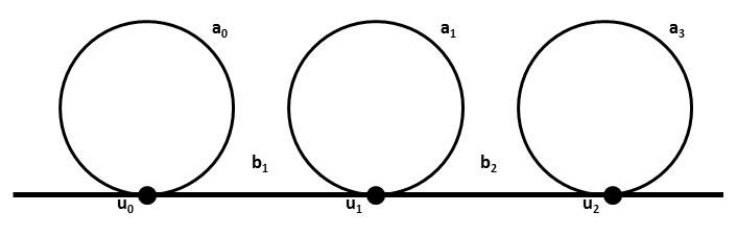

Figura B.2: Figura esquemática do procedimento de recorrência em um modelo de cadeia unidimensional. O conjunto $\left\{a_{n}\right\}$ corresponde a energias de um sítio onde o conjunto $\left\{b_{n}\right\}$ descrevem o hopping entre os estados.

$u_{n-1}$ e $u_{n+1}$. Definindo um conjunto $\left\{a_{0}, a_{1}, \ldots, a_{N}\right\}$ que descrevem a energia do sítio e $\left\{b_{1}, b_{2}, \ldots, b_{N}\right\}$ para descrever os parâmetros de hopping entre os estados. Podemos escrever a Hamiltoniana como:

$$
H u_{n}=a_{n} u_{n}+b_{n+1} u_{n+1}+b_{n-1} u_{n-1} .
$$

A relação B.1 é simétrica devido a componente de $u_{n+1}$ de $H u_{n}$ é idêntico a $u_{n}$ de $H u_{n+1}$. Isto é uma consequência do fato da Hamitoniana ser hermitiana. Representando os estados $u_{n}$ como um vetor coluna podemos escrever 
a Hamiltoniana em uma forma matricial da seguinte forma:

$$
H=\left[\begin{array}{ccccc}
a_{0} & b_{1} & 0 & 0 & \ldots \\
b_{1} & a_{1} & b_{2} & 0 & \ldots \\
0 & b_{2} & a_{2} & b_{3} & \ldots \\
0 & 0 & b_{3} & a_{3} & \ldots \\
\vdots & \vdots & \vdots & \vdots & \ddots
\end{array}\right]
$$

O conjunto $a_{n}$ e $b_{n}$ são chamados de parâmetros de recorrência e são usados para a construção de fração continuada que são usados no cálculo da densidade de estados local para um orbital inicial.

Os parâmetros de recorrência são obtidos escolhendo o orbital inicial, $\left|u_{0}\right\rangle$ de acordo com os nossos objetivos (orbital de base associado ao sítio em que queremos calcular a densidade de estados local). As novas funções de base ortogonais, $\left|u_{n}\right\rangle$, são definidos pela Relação de Recorrência (eq. B.1) para $n=0$

$$
H\left|u_{0}\right\rangle=a_{0}\left|u_{0}\right\rangle+b_{1}\left|u_{1}\right\rangle .
$$

Multiplicando-se escalarmente por $\left\langle u_{0}\right|$ e usando a ortonormalidade, temos:

$$
a_{0}=\left\langle u_{0}|H| u_{0}\right\rangle \text {. }
$$

Obtido $a_{0}$, pode-se calcular $b_{1}$ :

$$
b_{1}\left|u_{1}\right\rangle=\left(H-a_{0}\right)\left|u_{0}\right\rangle
$$

multiplicando por seu complexo conjugado

$$
\begin{aligned}
\left\langle u_{1}\left|b_{1}^{2}\right| u_{1}\right\rangle & =\left\langle u_{0}\left|\left(H-a_{0}\right)^{\dagger}\left(H-a_{0}\right)\right| u_{0}\right\rangle \\
b_{1} & =\left\langle u_{0}\left|\left(H-a_{0}\right)^{\dagger}\left(H-a_{0}\right)\right| u_{0}\right\rangle^{1 / 2} .
\end{aligned}
$$

Determinando $b_{1}$, podemos calcular $\left|u_{1}\right\rangle$

$$
\left|u_{1}\right\rangle=\frac{\left(H-a_{0}\right)}{b_{1}}\left|u_{0}\right\rangle .
$$

Com os valores de $a_{0}, b_{1}$ e $\left|u_{1}\right\rangle$ podemos calcular $a_{1}, b_{2}$ e $\left|u_{2}\right\rangle$, e com estes obtemos $a_{2}, b_{3}$ e $\left|u_{3}\right\rangle$ e assim por diante. Portanto, de maneira análoga, para qualquer $n$ :

$$
a_{n}=\left\langle u_{n}|H| u_{n}\right\rangle
$$




$$
\begin{gathered}
b_{n+1}^{2}=\left[\left\langleu_{n}\left|\left(H-a_{n}\right)^{\dagger}-\left\langle u_{n-1}\right| b_{n}^{\dagger}\right]\left[\left(H-a_{n}\right)\left|u_{n}\right\rangle-b_{n}\left|u_{n-1}\right\rangle\right]\right.\right. \\
\left|u_{n+1}\right\rangle=\frac{\left(H-a_{n}\right)\left|u_{n}\right\rangle-b_{n}\left|u_{n-1}\right\rangle}{b_{n+1}} .
\end{gathered}
$$

As componentes da Hamiltoniana, na nova base, serão dadas por:

$$
\begin{gathered}
H_{m, n}=\left\langle u_{m}|H| u_{m}\right\rangle=\left\langle u_{m}\left|a_{n}\right| u_{n}\right\rangle+\left\langle u_{m}\left|b_{n+1}\right| u_{n}\right\rangle+\left\langle u_{m}\left|b_{n}\right| u_{n}\right\rangle \\
H_{m, n}=\left\langle u_{m}|H| u_{n}\right\rangle=a_{n} \delta_{m, n}+b_{n+1} \delta_{m, n+1}+b_{n} \delta_{m, n-1}
\end{gathered}
$$

ou ainda na forma matricial (matriz tridiagonal, onde apenas os elementos da diagonal principal, e também da diagonal acima e abaixo da principal são não nulas)

$$
H=\left[\begin{array}{ccccc}
a_{0} & b_{1} & 0 & 0 & \ldots \\
b_{1} & a_{1} & b_{2} & 0 & \ldots \\
0 & b_{2} & a_{2} & b_{3} & \ldots \\
0 & 0 & b_{3} & a_{3} & \ldots \\
\vdots & \vdots & \vdots & \vdots & \ddots
\end{array}\right] .
$$

A partir das relações acima, os orbitais $\left|u_{n}\right\rangle$ são obtidos a partir de sucessivas aplicações de $\mathrm{H}$ a $\left|u_{0}\right\rangle$. Assim, à medida que $n$ cresce nos afastamos do sítio em que $\left|u_{0}\right\rangle$ está localizado, deste modo, diminuindo a influência do orbital $\left|u_{n+1}\right\rangle$ sobre $\left|u_{0}\right\rangle$. Portanto, para $n$ grande, o orbital se estende por uma região muito grande ( $\mathrm{n}+1$ vizinhos) e $\left|u_{n+1}\right\rangle$ não contribui no sítio de $\left|u_{0}\right\rangle$ tendo pouca importância em um cálculo de densidade de estados local nesse sítio. Assim, a transformação termina quando para um dado $N, b_{n+1}=0$. No entanto, para $n>L L$ (chamado de parâmetro de corte) as contribuições de $\left|u_{n}\right\rangle$ são muito pequenas e desprezamos os coeficientes a partir de $n=L L$. $\mathrm{O}$ valor escolhido para LL depende do tamanho do aglomerado e da precisão desejada. Para $n>L L$ a contribuição dos coeficientes $a_{n}$ e $b_{n}$ podem ser simulados através de um terminador [68]. Com isso resolvemos a primeira parte do problema.

O próximo passo é calcular a densidade de estados local a partir dos parâmetros $\left\{a_{n}\right\}$ e $\left\{b_{n}\right\}$. Entretanto, quando resolvemos o problema com o uso do parâmetro de corte obtemos um espectro discreto. Desta maneira, 
podemos tornar a densidade de estados contínua usando um terminador. Mostramos em seguida como obter uma densidade de estados contínua, a partir da Hamiltoniana tridiagonalizada fazendo uso da função de Green.

A densidade de estados local (LDOS) para orbital $\left|u_{0}\right\rangle$ é definida por:

$$
N_{0}(E)=L D O S=-\frac{1}{\pi} \operatorname{Im}\left\{G_{0}(E)\right\},
$$

onde $G_{0}(E)$ é o primeiro elemento da diagonal principal da matriz:

$$
G_{0}(E)=\left\langle u_{0}\left|(E-H)^{-1}\right| u_{0}\right\rangle
$$

com

$$
(E-H)^{-1}=\left[\begin{array}{ccccc}
\left(E-a_{0}\right) & -b_{1} & 0 & 0 & \ldots \\
-b_{1} & \left(E-a_{1}\right) & -b_{2} & 0 & \ldots \\
0 & -b_{2} & \left(E-a_{2}\right) & -b_{3} & \ldots \\
0 & 0 & -b_{3} & \left(E-a_{3}\right) & \ldots \\
\vdots & \vdots & \vdots & \vdots & \ddots
\end{array}\right]^{-1}
$$

O elemento de $A_{i j}$ da inversa de uma matriz $A$ é:

$$
A_{i j}=\frac{\operatorname{cof}(A)_{i j}}{\operatorname{det}(A)}
$$

onde $\operatorname{cof}(A)_{i j}$ é matriz cofatora de $A$ e $\operatorname{det}(A)$ é o determinante desta matriz. Assim, o elemento $(1,1)$ da matriz inversa é a razão do cofator de $(E-H)$ pelo determinante. Definindo $D_{n}(E)$ como determinante da matriz com as $n$ primeiras linhas e colunas, suprimidas, teremos:

$$
G_{0}(E)=\frac{D_{1}(E)}{D_{0}(E)}
$$

Usando a propriedade de determinantes de matriz $n \times n$ :

$$
\operatorname{det} A_{n \times n}=\sum_{1}^{n}(-1)^{i+1} A_{i 1}, D_{i, 1},
$$

onde $D_{i, 1}$ é o determinante da matriz $A$ com a linha $i$ e a coluna 1 suprimida, portanto:

$$
D_{0}(E)=(-1)^{1+1}\left(E-a_{0}\right) D_{1,1}+(-1)^{2+1}\left(-b_{1}\right) D_{2,1}
$$


$\operatorname{com} D_{1,1}=D_{1}(E) \mathrm{e}$

$$
D_{2,1}=(-1)^{2}\left(-b_{1}\right) D_{2}(E)
$$

e

$$
D_{0}(E)=\left(E-a_{0}\right) D_{1}(E)-b_{1}^{2} D_{2}(E) .
$$

Usando a definição da função de Green (eq. B.13)

$$
G_{0}=\frac{D_{1}(E)}{D_{0}(E)}=\frac{1}{D_{0} / D_{1}(E)}=\frac{1}{E-a_{0}-b_{1}^{2} D_{2}(E) / D_{1}(E)}
$$

podemos escrever:

$$
D_{1}(E)=\left(E-a_{1}\right)(-1)^{2} D_{2}(E)-\left(-b_{2}\right)^{2} D_{3}(E)
$$

ou genericamente

$$
D_{n}(E)=\left(E-a_{n}\right) D_{n+1}(E)-\left(-b_{n+1}\right)^{2} D_{n+2}(E) .
$$

Desta forma, verificamos que $G_{0}(E)$ pode ser expresso pela fração continuada

$$
G_{0}(E)=\frac{1}{\left(E-a_{0}\right)-\frac{b_{1}^{2}}{\left(E-a_{1}\right)-\frac{b_{2}^{2}}{\left(E-a_{2}\right)-\frac{b_{3}^{2}}{E-a_{3}-\cdots}}}} .
$$

Esta fração continuada pode ser finalizada em certo ponto gerando um espectro discreto, ou continuar indefinidamente tendo-se um espectro contínuo. Como queremos um espectro contínuo, truncamos a fração em um determinado ponto e usamos um terminador para representar os termos eliminados. Neste caso escrevemos a fração na seguinte forma:

$$
G_{0}(E)=\frac{1}{\left(E-a_{0}\right)-\frac{b_{1}^{2}}{\left(E-a_{1}\right)-\frac{b_{2}^{2}}{\left(E-a_{2}\right)-\frac{b_{3}^{2}}{\ddots \cdot \frac{b_{L L}}{E-a_{L L-1}-\frac{b_{L L}^{2}}{\left(E-a_{L L}\right)-t(E)}}}}}} .
$$


Nesta equação $t(E)$ é chamado de terminador da fração continuada e representa a contribuição dos termos para $n>L L$. Num procedimento descrito por Beer e Pettifor [68], toma-se $a_{n}=a$ e $b_{n}=b$ constantes para $n>L L$ e como a fração é infinita pode-se escrever:

$$
t(E)=\frac{b^{2}}{E-a_{N}-t(E)} .
$$

Portanto, para $\mathrm{t}(\mathrm{E})$ temos:

$$
[t(E)]^{2}-\left(E-a_{N}\right) t(E)+b_{N}^{2}=0
$$

a solução desta equação de segundo grau para t(E) é:

$$
\begin{gathered}
t(E)=\frac{1}{2}\left[\left(E-a_{N}\right) \pm \sqrt{\left(E-a_{N}\right)^{2}-4 b_{N}^{2}}\right] \\
t(E)=\frac{1}{2}\left[\left(E-a_{N}\right) \pm \sqrt{\left(E-a_{N}-2 b_{N}\right)\left(E-a_{N}+2 b_{N}\right)}\right] .
\end{gathered}
$$

A eq. B.28 quando substituída na fração continuada gera um espectro contínuo para a densidade de estados local dentro do intervalo:

$$
a_{L L}-2 b_{L L}<E<a_{L L}+2 b_{L L} .
$$

Repetindo esse processo para todos os orbitais centrados no mesmo sítio e somando suas contribuições, ou seja, somando as densidades de estados de todos os orbitais, encontramos a densidade de estados total para este sítio.

No nosso caso, para o cálculo da densidade de estados por sítio $R$, escolhese cada uma das funções de base $\left|u_{0}\right\rangle=\left|\chi_{R L}^{0}\right\rangle$ como o estado inicial, e construindo 9 cadeias obtemos a densidade total de estados como a soma dessas 9 contribuições. 


\section{Lista de Figuras}

2.1 Fluxograma relativo ao esquema da parte geral do método RS-LMTO-ASA. . . . . . . . . . . . . . . . . . 12

2.2 Fluxograma relativo ao esquema da parte atômica do método RS-LMTO-ASA. . . . . . . . . . . . . . . . . . 14

2.3 Esquema para a superfície livre com duas camadas de esferas vazias (ESF-1, ESF-2) e com cinco camadas metálicas $(\operatorname{MET}(\mathrm{S}), \operatorname{MET}(\mathrm{S}-1), \operatorname{MET}(\mathrm{S}-2), \operatorname{MET}(\mathrm{S}-3)$ e $\operatorname{MET}(\mathrm{S}-4))$ depositadas sobre o material bulk metálico. . . . . . . . . . 15

2.4 Esquema para o cálculo autoconsistente para o caso do adátomo. 18

3.1 Exemplos de ordenamentos magnéticos: a) ferromagnético (FM), b) antiferromagnético (AF) e c) não colinear. . . . . . . . . . 20

3.2 Ilustração de uma rede de skyrmions que pode ser vista como um redemoinho de momentos magnéticos. . . . . . . . . . . . 22

3.3 Ilustração de átomos magnéticos colocados em um triângulo eqüilátero: (a) solução FM, (b) solução AF frustrada e (c) solução não colinear onde os momentos fazem um ângulo de $120^{0}$ com os vizinhos. . . . . . . . . 24

3.4 Esquema mostrando a competição da interação de troca FM e AF entre os momentos magnéticos dos átomos da nanoestrutura e do substrato: (a) dímero AF, (b) substrato FM, (c) dímero depositado em um substrato FM e (d) devido a competição entre as interações de troca ocorre uma estrutura magnética não colinear no dímero.

4.1 Densidade de estados local (LDOS) do Fe metálico. A linha vertical passando por zero refere-se ao nível de Fermi. . . . . . 33 
4.2 Representação esquemática para o arranjo atômico de uma

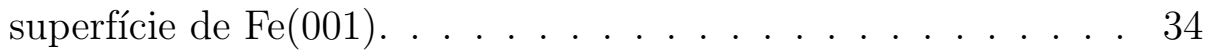

4.3 Densidade de estados local para os átomos de Fe nas camadas $\mathrm{Fe}(\mathrm{S}), \mathrm{Fe}(\mathrm{S}-1), \mathrm{Fe}(\mathrm{S}-2)$ e Fe(S-3) da superfície Fe(001). . . . . 37

4.4 Ilustração da primeira (a) e segunda (b) vizinhanças de um adátomo Mn sobre o $\mathrm{Fe}(001)$. Note-se que no sistema (b) têm-se quatro Fe1(S) e um Fe2(S-1). . . . . . . . . . . . . 38

4.5 Configurações: a) FM e b) AF para o adátomo Mn sobre a superfície $\mathrm{Fe}(001) \ldots \ldots \ldots \ldots$. . . . . . . . . . . . . 40

4.6 Representação esquemática para a impureza de Mn embebida na primeira camada de $\mathrm{Fe}(001) . \quad$. . . . . . . . . . . . . 41

4.7 Representação esquemática ilustrando a impureza Mn e seus primeiros, [Fe1(S-1)], e segundos vizinhos, [Fe2(S) e Fe2(S-2)]. Note-se que no sistema presente têm-se quatro Fe1(S-1), quatro Fe2(S) e um

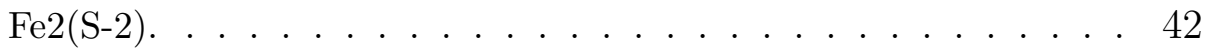

4.8 Densidades de Estados Locais projetados nos sítios de Mn: (a) adátomo FM e (b) impureza $\mathrm{AF}$ em $\mathrm{Fe}(001))$. . . . . . . . . 43

4.9 Ilustração das cadeias lineares finitas aqui estudadas contendo (a) 2, (b) 3, (c) 4, (d) 5 e (e) 9 átomos de Mn adsorvidos na superfície $\mathrm{Fe}(001)$ : átomos de $\mathrm{Mn}$ em vermelho e átomos de Fe em cinza. . . . . . . . . . . . . . . . . . . . 44

4.10 Ilustração das diferentes configurações magnéticas colineares usadas neste trabalho para os nanofios de Mn em Fe(001): (a) FM, (b) AF, (c) FI1, (d) FI2 e (e) FI3. . . . . . . . . . . . . . 45

4.11 Ilustração de um nanofio $\mathrm{Mn}_{5} / \mathrm{Fe}(001)$, onde são ilustrados os átomos de $\mathrm{Fe}$ com um primeiro $(\mathrm{Fe} 1 \mathrm{~A})$ e dois primeiros vizinhos (Fe1B) Mn. Também são ilustrados os átomos de Fe segundos vizinhos (esferas amarelas) do Mn. . . . . . . . . . . . . 45

4.12 Dímero e trímero de Mn depositados em forma diagonal na superfície $\mathrm{Fe}(001) \ldots \ldots \ldots \ldots$. . . . . . . . . . 46 
4.13 Acoplamentos de troca $J_{i j}$ em função da distância envolvendo os nanofios de $\mathrm{Mn}_{n} / \mathrm{Fe}(001)$ : (a) entre os momentos magnéticos dos átomos de Mn, $\mathrm{J}_{M n-M n}$, e (b) átomos de Mn e Fe, $\mathrm{J}_{M n-F e} 48$

4.14 Configurações magnéticas obtidas para: (a) dímero e (b) trímero, onde um átomo de Mn é embebido na primeira camada de Fe e os outros (um ou dois átomos de Mn) são colocados como adátomos. . . . . . . . . . . . . . . . 50

4.15 Configurações magnéticas não colineares para os nanofios: (a) $\mathrm{Mn}_{3}$, (b) $\mathrm{Mn}_{4}$, (c) $\mathrm{Mn}_{5}$, e (d) $\mathrm{Mn}_{9}$ em $\mathrm{Fe}(001)$. Configuração magnética para o nanofio $\mathrm{Mn}_{9} \operatorname{sem}(\mathrm{e})$ e com (f) acoplamento spin-órbita. Em (f) as setas são proporcionais ao momento magnético local. . . . . . . . . . . . . . . . . . 52

4.16 Configurações magnéticas não colineares obtidas para os sistemas com Mn embebido em $\mathrm{Fe}(001)$ : a) dímero e b) trímero de Mn. . . . . . . . . . . . . . . . . . 53

4.17 Configurações magnéticas para ilhas de Mn com (a) quatro (quadrado), (b) seis (retângulo), e (c) cinco (cruz) átomos em $\mathrm{Fe}(001)$. Os estados colineares são mostrados em (a1), (b1), e (c1) e seus correspondentes estados não colineares em (a3), (b3) e (c3). As esferas em azul em (c3) indicam os átomos de Fe próximos ao Mn central. . . . . . . . . . . . . 55

4.18 Configurações magnéticas para o aglomerado de forma piramidal adsorvido em Fe(001). As setas são proporcionais aos momentos magnéticos locais. . . . . . . . . . . . 55

4.19 Interação de troca $J_{i j}$ entre pares calculados como função da distância interatômica $d$ para diferentes ilhas de Mn adsorvidas em Fe(001) apresentadas na Fig. 4.17: (a) $\mathrm{J}_{M n-M n}$ e (c) $\mathrm{J}_{M n-F e}$. O gráficos (b) mostra os valores de $J_{M n-M n}$ entre sítios primeiros vizinhos em função de $N_{M n}$ (número de primeiros mais segundos vizinhos $\mathrm{Mn}$ ). O gráfico (d) mostra os valores de $J_{M n-F e}$ entre sítios primeiros vizinhos em função do número de primeiros vizinhos Mn. . . . . . 57 
4.20 Interação de troca magnética como função da distância entre os sítios para os átomos mostrados na Fig. 4.18: (a) $J_{M n-M n}$ e (b) $J_{M n-F e} \ldots \ldots \ldots \ldots \ldots$

5.1 Cadeias lineares finitas de átomos de Mn adsorvidas em uma superfície Fe(110): (a) adátomo, (b) dímero, (c) trímero, (d) $\mathrm{Mn}_{4}$, (e) $\mathrm{Mn}_{5}$ e (f) $\mathrm{Mn}_{6}$. As esferas vermelhas e cinzas indicam os átomos de Mn e Fe, respectivamente. . . . . . . . . . . 61

5.2 Ilustração do (a) adátomo e (b) impureza de Mn sobre o Fe(110). Note-se que existem dois primeiros vizinhos Fe para o adátomo, enquanto a impureza tem seis primeiros vizinhos Fe. . . . . . . 62

5.3 Densidade de Estados Local projetado no sítio de (a) adátomo e (b) impureza de Mn em $\mathrm{Fe}(110) \ldots$. . . . . . . . . . . . . . . 63

5.4 Interações de troca magnética em função da distância entre (a) átomos de $\mathrm{Mn}, \mathrm{J}_{M n-M n}$, e (b) átomos de $\mathrm{Mn}$ e Fe, $\mathrm{J}_{M n-F e}$.

5.5 Configurações magnéticas não colineares para nanofios com (a) $\mathrm{Mn}_{2}$, (b) $\mathrm{Mn}_{3}$, (c) $\mathrm{Mn}_{4}$, (d) $\mathrm{Mn}_{5}$, and (e) $\mathrm{Mn}_{6}$ em Fe(110).

6.1 Ilustração das três partes de um dispositivo de gravação magnética. 69

6.2 Ilustração do nanofio de (a) Co puro e (b) $\mathrm{Fe}_{0,11} \mathrm{Co}_{0,89}$ depositados em Pt(111), considerando os arranjos possíveis neste caso. 72

6.3 Cadeias lineares finitas de $\mathrm{Fe}_{x} \mathrm{Co}_{1-x}$ adsorvidas em uma superfície $\mathrm{Pt}(111)$ : a) $\mathrm{Fe}_{1,0} \mathrm{Co}_{0,0}$, b) $\mathrm{Fe}_{0,56} \mathrm{Co}_{0,44}$ e c) $\mathrm{Fe}_{0,22} \mathrm{Co}_{0,78}$. As esferas vermelhas, rosas e cinzas indicam os átomos de $\mathrm{Fe}$, Co e Pt, respectivamente. . . . . . . . . . . . . 73

6.4 Comportamento dos momentos magnéticos (a) $\mathrm{m}_{s}$ e (b) $\mathrm{m}_{L}$ dos átomos de $\mathrm{Fe}$ e $\mathrm{Co}$ em nanofios de $\mathrm{Fe}_{x} \mathrm{Co}_{1-x}$ sobre superfície

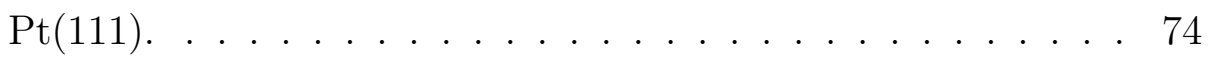

6.5 Comportamento do momento magnético dos primeiros vizinhos Pt dos nanofios de $\mathrm{Fe}_{x} \mathrm{Co}_{1-x}$ sobre a superfície $\mathrm{Pt}(111)$ em função de $x \ldots \ldots \ldots \ldots \ldots \ldots \ldots$ 
6.6 Comportamento dos momentos magnéticos orbitais em função de $x$ para nanofios de $\mathrm{Fe}_{x} \mathrm{Co}_{1-x}$ depositados sobre $\mathrm{Pt}(111)$ :

a) com OP e b) sem OP. . . . . . . . . . . . . . . 78

6.7 Densidades de estados locais projetadas nos sítios de Fe e Co localizados na posição interna da cadeia linear (a) e (b) na extremidade da cadeia linear (c) e (d) em função de diferentes tipos de vizinhos. 80

B.1 Figura esquemática dos estados expandidos no espaço nas vizinhanças do átomo central. Aqui o estado inicial $u_{0}$ é o circulo preto, seus vizinhos são representados por $u_{1}$ (esfera cinza) e $u_{2}$ (esfera amarela). . . . . . . . . . . . . . 101

B.2 Figura esquemática do procedimento de recorrência em um modelo de cadeia unidimensional. O conjunto $\left\{a_{n}\right\}$ corresponde a energias de um sítio onde o conjunto $\left\{b_{n}\right\}$ descrevem o hopping entre os estados. . . . . . . . . . . . . . 101 


\section{Bibliografia}

[1] M. N. Babich, J. M. Broto, A. Fert, F. N. V. Dau, F. Petroff, P. Eitenne, G. Creuzet, A. Friedrich, and J. Chanzelas, Phys. Rev. Lett. 61, 2472 (1988).

[2] A. P. Guimarães, Principles of Nanomagnetism (Springer, Berlin, 2009).

[3] M. Menzel, Y. Mokrousov, R. Wieser, J. E. Bickel, E. Vedmedenko, S. Blügel, S. Heinze, K. von Bergman, A. Kubetzka, and R. Wiesendanger, Phys. Rev. Lett 108, 197204 (2012).

[4] M. Heide, G. Bihlmayer, and S. Blügel, Physica B 404, 2678 (2009).

[5] T. Schulz, R. Ritz, A. Bauer, M. Halder, M. Wagner, C. Franz, C. Pfleiderer, K. Everschor, M. Garst, and A. Rosch, Nat. Phys. 8, 301 (2012).

[6] S. Heinze, K. von Bergmann, M. Menzel, J. Brede, A. Kubetzka, R. Wiesendanger, G. Bihlmayer, and S. Blügel, Nat. Phys. 7, 713 (2011).

[7] T. Seiki, Y. Hasegawa, S. Mitani, S. Takahasi, H. Imamura, S. Maekawa, J. Nitta, and K. Takanashi, Nat. Mater. 7, 125 (2008).

[8] J. M. Vargas, R. D. Zysler, L. M. Socolovsky, M. Knobel, and D. Zanchet, J. Appl. Phys. 101, 023903 (2007).

[9] P. Gambardella, S. Rusponi, M. Veronese, S. S. Dhesi, C. Grazioli, A. Dallmeyer, I. Cabria, R. Zeller, P. H. Dederichs, K. Kern, C. Carbone, and H. Brune, Science 300, 1130 (2003). 
[10] P. Gambardella, J. Phys. Condens. Matter 15, S2533 (2003).

[11] P. Gambardella, A. Dallmeyer, K. Maiti, M. C. Malagoli, W. Eberhardt, K. Kern, and C. Carbone, Nature 416, 301 (2002).

[12] I. Zutic, J. Fabian, and S. Das Sarma, Rev. Mod. Phys. 76, 323 (2004).

[13] P. Weinberg, Phys. Rev. B 75, 064405 (2007).

[14] C. F. Hirjibehedin, C. P. Lutz, and A.J. Heinrich, Science 312, 1021 (2006).

[15] F. Meier, L. Zhou, J. Wiebe, and R. Wiesendanger, Science 320, 82 (2008).

[16] C. Grazioli, D. Alfè, S. R. Krishnakumar, S. S. Gupta, M. Veronese, S. Turchini, N. Bonini, A. Dal Corso, D. D. Sarma, S. Baroni, and C. Carbone, Phys. Rev. Lett. 95, 107201 (2005).

[17] J. Dresselhaus, D. Spanke, F. U. Hillebrecht, E. Kisker, G. van der Laan, J. B. Goedkoop, and N. B. Brookes, Phys. Rev. B 56, 5461 (1997).

[18] O. Rader, W. Gudat, D. Schmitz, C. Carbone, and W. Eberhardt, Phys. Rev. B 56, 5053 (1997).

[19] Ch. Roth, Th. Kleeman, F. U. Hillebrecht, and E. Kisker, Phys. Rev. B 52, R15691 (1995).

[20] T. K. Yamada, E. Martínez, A. Vega, R. Robles, D. Stoeffler, A. L. Vázquez de Parga, T. Mizoguchi, and H. van Kempen, Nanotechnology 18,235702 (2007).

[21] D. Spisák and J. Hafner, Surf. Sci. 601, 4348 (2007).

[22] J. Hafner and D. Spisák, Phys. Rev. B 72, 144420 (2005).

[23] T. Asada, S. Blügel, G. Bihlmayer, S. Handschuh, and R. Abt, J. Appl. Phys. 87, 5935 (2000). 
[24] S. Handschuh and S. Blügel, Solid State Commun. 105, 633 (1998).

[25] O. Elmouhssine, G. Moraitis, C. Demangeat, and J. C. Parlebas, Phys. Rev. B 55, R7410 (1997).

[26] D. Spisak and J. Hafner, Phys. Rev. B 55, 8304 (1997).

[27] S. Mirbt, O. Eriksson, B. Johansson, and H. L. Skriver, Phys. Rev. B $52,15070(1995)$.

[28] E. Martínez, A. Veja, R. Robles, and A. L. Vázquez de Parga, Phys. Lett. A 337, 469 (2005).

[29] R. Wu and A. J. Freeman, Phys. Rev. B 51, 17131 (1995).

[30] B. Nonas, K. Wildberger, R. Zeller, and P. H. Dederichs, Phys. Rev. Lett. 80, 4574 (1998); J. Magn. Magn. Mater. 165, 137 (1997).

[31] J. T. Lau, A. Fohlisch, R. Nietubye, M. Reif, and W. Wurth, Phys. Rev. Lett. 89, 057201 (2002).

[32] S. Rusponi, T. Cren, N. Weiss, M. Epple, P. Buluschek, L. Claude, and H. Brune, Nat. Mater. 2, 546 (2003).

[33] W. Kuch, Nat. Mater. 2, 505 (2003).

[34] J. A. Stroscio and R. J. Celotta, Science 306, 242 (2004).

[35] Y. Yayon, V. W. Brar, L. Senapati, S. C. Erwin, and M. F. Crommie, Phys. Rev. Lett. 99, 067202 (2007).

[36] T. Balashov, T. Schuh, A. F. Takács, A. Ernst, S. Ostanin, J. Henk, I. Mertig, P. Bruno, T. Miyamachi, S. Suga, and W. Wulfhekel, Phys. Rev. Lett. 102, 257203 (2009).

[37] A. J. Heinrich, J. A. Gupta, P. Lutz, and D. M. Eigler, Science 306, 466 (2004).

[38] W. Kohn and L. J. Shan, Phys. Rev. 140, 1133 (1965). 
[39] P. R. Peduto, S. Frota-Pessôa and M.l S. Methfessel, Phys. Rev. B 44, 13283 (1991); J. Duarte Jr, P. R. Peduto, and S. Frota-Pessôa, Rev. Bras. Fis. 21, 101 (1991); S. Frota-Pessôa, Phys. Rev. B 46, 14570 (1992); H. M. Petrilli and S. Frota-Pessôa, Phys. Rev. B 48 (1993).

[40] R. Robles, A. Bergman, A. B. Klautau, O. Eriksson, and L. Nordströn, J. Phys. Condens. Matter 20, 015001 (2008).

[41] A. Bergman, L. Nordströn, A. B. Klautau, S. Frota-Pessôa, and O. Eriksson, J. Magn. Magn. Mater. 320, 1173 (2008); Phys. Rev. B 73, 174434 (2006); Phys. Rev. B 75, 224425 (2007); Surf. Sci. 600, 4838 (2006).

[42] A. B. Klautau and S. Frota-Pessôa, Surf. Sci. 579, 27 (2005) A. B. Klautau and S. Frota-Pessôa, Phys. Rev. B 70, 193407 (2004); S. FrotaPessôa, Phys. Rev. B 69, 104401 (2004); W. D. Brewer, A. Scherz, C. Sorg, H. Wende, K. Baberschke, P. Bencok, and S. Frota-Pessôa, Phys. Rev. Lett. 93, 077205 (2004).

[43] A. T. Costa Jr., R. B. Muniz, and D. L. Mills, Phys. Rev. Lett. 94, 137203 (2005).

[44] N. N. Negulyaev, V. S. Stepanyuk, W. Hergert, and J. Kirschner, Phys. Rev. Lett. 106, 037202 (2011).

[45] H. Tan, E. Martínez, A. Vega, V. M. Uzdin, R. Robles, and G. Borstel, Surf. Sci. 603, 2537 (2009).

[46] R. N. Igarashi, A. B. Klautau, R. B. Muniz, B. Sanyal, and H. M. Petrilli, Phys. Rev. B 85, 014436 (2012).

[47] O. Rader, C. Pampuch, W. Gudat, A. Dallmeyer, C. Carbone, and W. Eberhardt, Europhys. Lett. 46, 231 (1999).

[48] D. I. Bardos, J. Appl. Phys. 40, 1371 (1969). 
[49] I. Turek, J. Kudrnovský, V. Drchal, and P. Weinberger, Phys. Rev. B 49, 3352 (1994).

[50] P. Söderlind, O. Eriksson, B. Johansson, R. C. Albers, and A. M. Boring, Phys. Rev. B 45, 12911 (1992).

[51] L. Pauling, Phys. Rev. 54, 899 (1938).

[52] T. Burkert, L. Nordstron, O. Eriksson, and O. Heinonen, Phys. Rev. Lett. 93, 027203 (2004).

[53] G. Moulas, A. Lehnert, S. Rusponi, J. Zabloudil, C. Etz, S. Ouazi, M. Etzkorn, P. Bencok, P. Gambardella, P. Weinberger, and H. Brune, Phys. Rev. B 78, 214424 (2008).

[54] C. Neise, S. Schönecker, M. Richter, K. Koepernik, and H.Eschrig, Phys. Status Solidi B 248, 2398 (2011).

[55] F. Yildiz, F. Luo, C. Tieg, R. M. Abrudan, X. L. Fu, A. Winkelmann, M. Przybylski, and J. Kirschner, Phys. Rev. Lett. 100, 037205 (2008).

[56] C. Etz, B. Lazarovits, J. Zabloudil, R. Hammerling, B. Újfalussy, L. Szunyogh, G. M. Stocks, and P. Weinberger, Phys. Rev. B 75, 245432 (1991).

[57] M. Born and J. R. Oppenheimer, Ann. Phys. 87, 457 (1927).

[58] P. Hohenberg and W. Kohn, Phys. Rev. Lett. 24, 58 (1970).

[59] U. von Barth and L. Hedin, J. Phys. C5, 1629 (1972).

[60] J. P. Perdew, K. Burke, and M. Ernzerhof, Phys. Rev. Lett. 77, 3865 (1996).

[61] O. K. Andersen, Phys. Rev. B. 12, 3060 (1975).

[62] D. D. Koelling and B. N. Harmon, J. Phys. C: Solid State Phys. 10, 3107 (1977). 
[63] M. S. S. Brooks and P. J. Kelly, Phys. Rev. Lett. 51, 1708 (1983).

[64] O. Eriksson, M. S. S. Brooks, and B. Johanssoon, Phys. Rev. B 41, 7311 (1990); M. S. S. Brooks, Physica B 130, 6 (1985); O. Eriksson, B. Johansson, R. C. Albers, A. M. Boring, and M. S. S. Brooks, Phys. Rev. B 42, 2707 (1990).

[65] O. K. Andersen, O. Josepsen and O. Goetzel, Highlights of condensed matter theory (Worth-Holland, Amsterdan, 1985).

[66] O. K. Andersen and O. Jepsen, Phys. Rev. Lett. 53, 2571 (1984).

[67] R. Haydock, Solid State Physics (Acad. Press, New York, 1980).

[68] N. Beer and D. Pettifor, The Electronic Structure of Complex Systems (Plenum Press, New York, 1984).

[69] A. B. Kautau and S. Frota-Pessôa, Int. J. Mod. Phys. B 20, 5281 (2006).

[70] S. Ferreira, Parametrização LMTO-ASA no cálculo de estrutura eletrônica de metais de transição. Dissertação de mestrado apresentada no IFUSP (1989).

[71] A. B. Klautau, Cálculos Ab Initio da Estrutura Eletrônica e Propriedades Magnética de Sistemas Metálicos Bidimensionais. Tese de doutorado apresentada no IFUSP (2000).

[72] R. N. Nogueira, Estudo de Propriedades Locais nas Impurezas de Fe ou Co em Ligas de Nb e Mo. Dissertação de mestrado apresentada no IFUSP (1995).

[73] H. L. Skriver and N. M. Rosengaard, Phys. Rev. B 43, 9538 (1991).

[74] M. Aldém, S. Mirbt, H. L. Skriver, N. M. Rosenggard, and B. Johansson, Phys. Rev. B 46, 6303 (1992); Surf. Sci. 315, 157 (1994).

[75] J. Stöhr and H. C. Siegmann, Magnetism From Fundamentals to Nanoscale Dynamics (Springer, Berlin, 2006). 
[76] N. W. Ashcroft and N. D. Mermim, Solid State Physics (Saunders College Publishing, Florida, 1976).

[77] A. Bergman, A Theoretical Study of Magnetism in Nanostructured Materials. Tese de doutorado apresentada na Uppsala University - Suécia (2006).

[78] I. S. Oliveira, V. L. B. de Jesus, Introdução À Fúsica do Estado Sólido (Livraria da Física, São Paulo, 2005).

[79] M. A. Ruderman and C. Kittel, Phys. Rev. 96, 99 (1954).

[80] T. Kasuya, Prog. Theor. Phys. 16, 45 (1956).

[81] K. Yosida, Phys. Rev. 106, 893 (1957).

[82] I. E. Dzyaloshinskii, J. Phys. Chem. Solids 4, 241 (1958).

[83] T. Moriya, Phys. Rev. 120, 91 (1960).

[84] U. K. Rößler, A. N. Bogdanov, and C. Pfleiderer, Nature 442, 797 (2006).

[85] A. I. Liechtenstein, M. I. Katsnelson, V. P. Antropov, and V. A. Gubanov, J. Magn. Magn. Mater. 67, 65 (1987)

[86] A. Oswald, R. Zeller, P. J. Braspenning, and P. H. Dederichs, J. Phys. F: Met. Phys. 15, 193 (1985).

[87] S. Frota-Pessôa, R. B. Muniz, and J. Kudrnovsky, Phys. Rev. B 62, 5293 (2000).

[88] Ph. Kurz, G. Bihlmayer, K. Hirai, and S. Blügel, Phys. Rev. Lett. 86, 1106 (2001).

[89] S. Lounis, Ph. Mavropoulos, P. H. Dederichs, and S. Blügel, Phys. Rev. B 72,224437 (2005). 
[90] O. N. Mryasov, A. I. Liechtenstein, L. M. Sandratskii, and V. A. Gubanov, J. Phys. Condens. Matter 3, 7683 (1991).

[91] K. Knöpfle, L. M. Sandratskii, and J. Kübler, Phys. Rev. B 62, 5564 (2000).

[92] L. Nordströn and D. J. Singh, Phys. Rev. Lett. 76, 4420 (1996).

[93] R. Lizárraga, L. Nordström, L. Bergqvist, A. Bergman, E. Sjösdedt, P. Mohn, and O. Eriksson, Phys. Rev. Lett. 91, 107205 (2004).

[94] T. Oda, A. Pasquarello, and R. Car, Phys. Rev. Lett. 80, 3622 (1998);

D. Hobbs, G. Kresse, and J. Hafner, Phys. Rev. B 62, 11556 (2000).

[95] T. K. Yamada, M. M. J. Bischoff, G. M. M. Heijnen, T. Mizoguchi, and H. van Kempen, Phys. Rev. Lett. 90, 056803 (2003).

[96] U. Schlickum, N. Janke-Gilman, W. Wulfhekel, and J. Kirschner, Phys. Rev. Lett. 92, 107203 (2004).

[97] T. G. Walker and H. Hopster, Phys. Rev. B 48, R3563 (1993).

[98] D. Tulchinsky, J. Unguris, and R. J. Celotta, J. Magn. Magn. Mater. 212, 91 (2000).

[99] S. T. Purcell, M. T. Johnson, N. W. E. McGee, R. Coehoorn, and W. Hoving, Phys. Rev. B 45, 13064 (1992).

[100] S. K. Kim, Y. Tian, M. Montesano, F. Jona, and P. M. Marcus, Phys. Rev. B 54, 5081 (1996).

[101] S. Andrieu, M. Finazi, Ph. Bauer, H. Fischer, P. Lefevre, A. Traverse, K. Hricovini, G. Krill, and M. Piecuch, Phys. Rev. B 57, 1985 (1998).

[102] M. M. J. Bischoff, T. K. Yamada, A. J. Quinn, and H. van Kempen, Surf. Sci. 501, 155 (2002). 
[103] T. K. Yamada, M. M. J. Bischoff, T. Mizoguchi, and H. van Kempen, Surf. Sci. 516, 179 (2002).

[104] W. Wulfhekel, U. Schlickum, and J. Kirschner, Micros. Res. Tech. 66, 105 (2005).

[105] A. Tange, C. Gao, W. Wulfhekel, and J. Kirschner, Phys. Rev. B 81, 220404 (2010).

[106] P. Torelli, F. Sirotti, and P. Ballone, Phys. Rev. B 68, 205413 (2003).

[107] E. C. Passamani, B. Croonenborghs. B. Degroote, and A. Vantomme, Phys. Rev. B 67, 174424 (2003).

[108] V. L. Moruzzi, J. F. Janak, and A. R. Willians, Calculated Electronic Properties of Metal (Pergamon, New York, 1978).

[109] G. H. O. Daalperop, P. J. Kelly, and M. F. H. Schuurmans, Phys. Rev. B 41, 11919 (1990).

[110] K. Wagner, N. Weber, H. J. Elmers, and U. Gradmann, J. Magn. Magn. Mater. 167, 21 (1997).

[111] V. Popescu, H. Ebert, B. Nonas, and P. H. Dederichs, Phys. Rev. B 64, 184407 (2001).

[112] C. Binns, Surf. Sci. Rep. 44, 1 (2001).

[113] M. Ezawa, Phys. Rev. B 83, 100408 (2011).

[114] U . K. Rössler, A. N. Bogdanov, and C. Pfleidere, Nature 442, 797 (2006).

[115] G. E. Brown and M. Rho, Multifaceted Skyrmions (World Scientific, Singapore, 2010).

[116] X. Z. Yu, Y. Onose, N. Kanazawa, J. H. Park, J. H. Han, Y. Matsui, N. Nagaosa, and Y. Tokura, Nature 465, 901 (2010). 
[117] M. Bode, M. Heide, K. von Bergmann, P. Ferriani, S. Heinze, G. Bihlmayer, A. Kubetzka, O. Pietzsch, S. Blügel, and R. Wiesendanger, Nature 447, 190 (2007).

[118] S. Lounis, P. H. Dederichs, and S. Blugel, Phys. Rev. Lett. 101, 107204(2008).

[119] S. John, M. Berciu, and A. Golubentsev, Europhys. Lett. 41, 31 (1998).

[120] S. Heinze, K. von Bergmann, M. Menzel, L. Brede, A. Kubetzka, R. Wiesendanger, G. Bihlmayer, and S. Blügel, Nat. Phys. 7, 713 (2011).

[121] F. Schubert, Y. Mokrousov, P. Ferriani, and S. Heinze, Phys. Rev. B 83, 165442 (2011).

[122] M. Zelený, M. Sob, and J. Hafner, Phys. Rev. B 80, 144414 (2009).

[123] K. von Bergmann, S. Heinze, M. Bode, E. Y. Vedmedenko, G. Bihlmayer, S. Blügel, and R. Wiesendanger, Phys. Rev. Lett. 96, 167203 (2006).

[124] P. Mavropoulos, S. Lounis, and S. Blügel, Phys. Status Solidi B 247, 1187 (2010).

[125] M. S. Ribeiro, G. B. Corrêa Jr., A. Bergman, L. Nordströn, O. Eriksson, and A. B. Klautau, Phys. Rev. B 83, 014406 (2011).

[126] A. Moser, K. Takano, D. T. Margulies, M. Albrecht, Y. Sonobe, Y. Ilkeda, S. Sun, and E. F. Fullerton, J. Phys. D: Appl. Phys. 35, R157 (2002).

[127] J. S. Moodera, L. R. Kinder, T. M. Wong, and R. Meservey, Phys. Rev. Lett. 74, 3273 (1995).

[128] G. B. Corrêa Junior, Propriedades magnéticas de nanoestruturas de metais de transição 3d adsorvidas na superficie de Pt(111). Dissertação de mestrado apresentanda no ICEN-UFPA (2008). 
[129] M. S. Ribeiro, Configurações magnéticas colineares e não colineares em currais de Fe, Cr e Mn sobre a Pt(111). Dissertação de mestrado apresentanda no ICEN-UFPA (2010).

[130] M. M. Bezerra Neto, Propriedades magnéticas de nanoestruturas adsorvidas em superfícies metálicas. Dissertação de mestrado apresentanda no ICEN-UFPA (2011).

[131] S. Frota-Pessôa, Phys. Rev. B 69, 104401 (2004).

[132] Y. Tsunoda, J. Phys. Condens. Matter 1, 10427 (1989).

[133] Y. tsunoda, Y. Nishioka, and R. M. Nicklow, J. Magn. Magn. Mater. 128, 133 (1993).

[134] G. van der Laan, J. Phys. Condens. Matter 10, 3239 (1998).

[135] H. Ebert, R. Zeller, B. Drittler, and P. H. Dederichs, J. Appl. Phys. 67, 4576 (1990). 